\title{
ON TYPICALITY OF TRANSLATION FLOWS WHICH ARE DISJOINT WITH THEIR INVERSE
}

\author{
PRZEMYSŁAW BERK, KRZYSZTOF FRĄCZEK, AND THIERRY DE LA RUE
}

\begin{abstract}
In this paper we prove that translation structures for which the corresponding vertical translation flows is weakly mixing and disjoint with its inverse, form a $G_{\delta}$-dense set in every non-hyperelliptic connected component of the moduli space $\mathcal{M}$. This is in contrast to hyperelliptic case, where for every translation structure the associated vertical flow is isomorphic to its inverse. To prove the main result, we study limits of the offdiagonal 3-joinings of special representations of vertical translation flows. Moreover, we construct a locally defined continuous embedding of the moduli space into the space of measure-preserving flows to obtain the $G_{\delta}$-condition.
\end{abstract}

\section{INTRODUCTION}

Let $M$ be an orientable compact connected topological surface, and $\Sigma$ be a finite set of singular points. On $M$ we can consider a translation structure $\zeta$, i.e. an atlas on $M \backslash \Sigma$ such that every transition transformation is a translation. Every translation surface can be viewed as a polygon with pairwise parallel sides of the same length which are glued together (gluing is made by a translation). Parameters given by the sides of such polygons establish a parametrization of the so-called moduli space $\mathcal{M}$ and yield a topology on $\mathcal{M}$. To each translation structure $\zeta$ we associate the corresponding Lebesgue measure $\lambda_{\zeta}$ on $M$. Moreover, for every direction we consider the flow which acts by translation in that direction with unit speed. Such translation flows preserve $\lambda_{\zeta}$. In this paper we are interested in vertical translation flows. It is worth to mention that the study of directional flows on translation surfaces originates from problems concerning billiard flows on rational polygons (see [7, [14).

In [17 the authors give a complete characterisation of connected components of the moduli space; all of them are orbifolds. On each connected component $C$ we consider an action of $S L_{2}(\mathbb{R})$ which is derived form the linear action of $S L_{2}(\mathbb{R})$ on polygons. Moreover, there is a Lebesgue measure $\nu_{C}$ on $C$ which is invariant under this action. Let $\mathcal{M}_{1}$ be the set of $\zeta \in \mathcal{M}$ such that $\lambda_{\zeta}(M)=1$ and let $C_{1}:=C \cap \mathcal{M}_{1}$. We also consider a measure $\nu_{C_{1}}$ on $C_{1}$ which is a projectivization of $\nu_{C}$. This measure is finite and invariant under the action of $S L_{2}(\mathbb{R})$ on $C_{1}$. In fact, the action of $S L_{2}(\mathbb{R})$ is ergodic with respect to this measure (see [18, and [22]). This gives an opportunity to use the ergodic theory to study dynamical properties of vertical flows on almost all translations structures. In particular, it was used to prove that the sets of translation structures for which the vertical translation flow is ergodic (see [18]), and further is weakly mixing (see [3]) are of full measure in both $C$ and $C_{1}$. At the same time, there are no mixing translation flows (see 13]). In this paper we are interested in translation structures for which the corresponding vertical translation flow is disjoint with its inverse, which is a stronger notion than being not reversible. Recall that a measure preserving flow $\left\{T_{t}\right\}_{t \in \mathbb{R}}$ on $(X, \mu)$ is reversible, if there exists an involution $\theta: X \rightarrow X$ which preserves $\mu$ and

$$
\theta \circ T_{-t}=T_{t} \circ \theta \text { for all } t \in \mathbb{R} \text {. }
$$

Date: October 28, 2018

2000 Mathematics Subject Classification. 37A10, 37E35, 37C80.

Key words and phrases. measure-preserving flows, translation surfaces, reversibility of dynamical systems, joinings methods in ergodic theory. 
Our result concerns the topological typicality of the desired property rather than measuretheoretical. As a by-product, we give a method to show that the set of translation structures for which the associated vertical translation flows satisfy any property which is $G_{\delta}$ in the space of measure preserving flows, is also a $G_{\delta}$-set. Among these properties are for instance weak mixing, ergodicity and rigidity (see [12]).

In the classification of connected components given in [17] we distinguish so called hyperelliptic components. For every hyperelliptic component $C$ there exists an involution $\theta: M \rightarrow M$ such that for every $\zeta \in C$ it is given in local coordinates by the formula $z \mapsto-z+c$ for some $c \in \mathbb{C}$. In particular, the vertical flow on $(M, \zeta)$ is reversible; it is isomorphic with its inverse by the involution $\theta$ (see remark 2.8). In contrast, in this paper we show that on non-hyperelliptic components of the moduli space the set of translation structures for which the vertical flow is disjoint with its inverse is topologically large. It is expressed by the following theorem.

Theorem 1.1. Let $C$ be a non-hyperelliptic connected component of the moduli space of translation structures. Then the set of translation structures whose vertical flow is weakly mixing and disjoint with its inverse is a $G_{\delta}$-dense set in $C$.

It is also worth to mention that on non-hyperelliptic components we can also find a non-trivial set of translation structures for which the vertical flow is reversible.

Proposition 1.2. Let $C$ be a non-hyperelliptic connected component of the moduli space of translation structures. Then the set of translation structures whose vertical flow is reversible is dense in $C$.

Recall that a measure-preserving flow $\left\{T_{t}\right\}_{t \in \mathbb{R}}$ on a standard Borel probability space $(X, \mathcal{B}, \mu)$ is disjoint with its inverse if the only $\left(T_{t} \times T_{-t}\right)$ - invariant probability measure on $X \times X$, which projects on each coordinate as $\mu$ is the product measure $\mu \otimes \mu$. In $[9$ the authors developed techniques to prove non-isomorphism of a flow $T^{f}$ to its inverse that are based on studying the weak closure of off-diagonal 3-self-joinings. Moreover, in [4] the authors improved those techniques to show that a large class of special flows over interval exchange transformations and under piecewise absolutely continuous functions have the property of being non-isomorphic with their inverse. The idea of detecting non-isomorphism of a dynamical system and its inverse by studying the weak closure of off-diagonal 3-self-joinings was introduced by Ryzhikov in [20. In this paper we prove that techniques mentioned earlier can be used to detect disjointness of a vertical flow with its inverse.

To prove the $G_{\delta}$ condition, we use the result of Danilenko and Rhyzhikov from [5] (which derives from a version for automorphisms given in [6]), where they proved that the flows with the property of being disjoint with their inverse form a $G_{\delta}$-dense set in the space of measure preserving flows. To use their result we construct a locally defined continuous embedding of the moduli space into the space of measure preserving flows. To show the density condition, we largely rely on the proof of Lemma 14 in 8 .

In Section 2 we give a general background concerning joinings, interval exchange transformations, space of measure preserving flows, translation flows and moduli spaces. In particular, we give some tools needed to prove the continuity of a map with values in the space of flows and we state some connections between the moduli space and interval exchange transformations.

In Section 3 we introduce a criterion of disjointness of two flows by researching the weak limits of certain 3-self-joinings. This is a direct improvement of the criteria stated in [9] and [4] as we show that these are actually criteria of two weakly mixing flows being disjoint. Furthermore, we state a criterion of a flow being weakly mixing, which also uses weak limits of 3-self-joinings as a tool.

In Section 4 we state combinatorial conditions on translation structures which are later used in proving the density condition in our main theorem. To be precise, we show that our results apply to every non-hyperelliptic component of the moduli space.

In Section 5 we show that on a given translation surface $(M, \zeta)$ there exists $\varepsilon_{\zeta}>0$ such that for every absolutely continuous measure $\mu$ on $M$ which has density $f$ satisfying $\int_{M}|f(x)-1| d x<$ 
$\varepsilon_{\zeta}$ there exists a homeomorphism $H: M \rightarrow M$ such that $H_{*} \mu$ is the Lebesgue measure with $H$ depending continuously on $f$. The results of section 5 were inspired by the works of Moser in [19] and Goffman, Pedrick in [1].

In Section 6 we use the results presented in the previous section to construct a continuous embedding of each connected component of the moduli space into the space of measure-preserving flows. The embedding is defined locally, but we also show that this is enough to transfer the $G_{\delta}$ condition.

Finally, in Section 7 we first state a result which is a conclusion from the previous sections, that in every connected component of the moduli space the set of translation structures whose associated vertical translation flow is disjoint with its inverse, is a $G_{\delta}$ set. In the remainder of this section we use the results from [4 to show that the criteria introduced in Section 3 can be used for a dense set of translation structures in every non-hyperelliptic component, which leads to the proof of Theorem 1.1. As a by-product we get the proof of Proposition 1.2 .

\section{PRELiminaries}

We will now give some details regarding interval exchange transformations, joinings of dynamical systems and some basic information about moduli space.

2.1. Joinings. In this subsection we give some definitions which are stated for standard Borel probability spaces. However these definitions can be easily extended to standard Borel spaces with finite measures. Though here we state definitions for probability spaces, in the remainder of this paper we will freely use them in case when the measure is finite and not necessarily a probability. In particular we say that the measure preserving flows $\mathcal{T}=\left\{T_{t}\right\}_{t \in \mathbb{R}}$ on $(X, \mathcal{B}, \mu)$ and $\mathcal{S}=\left\{S_{t}\right\}_{t \in \mathbb{R}}$ on $(Y, \mathcal{C}, \nu)$ are isomorphic, if there exists a measurable $F:(X, \mathcal{B}) \rightarrow(Y, \mathcal{C})$ such that

$$
T_{t}=F^{-1} \circ S_{t} \circ F \text { for } t \in \mathbb{R} \quad \text { and } \quad F_{*} \mu=\frac{\mu(X)}{\nu(Y)} \nu .
$$

Let $K>0$ be a natural number and for $1 \leq i \leq K$ let $\mathcal{T}^{i}=\left\{T_{t}^{i}\right\}_{t \in \mathbb{R}}$ be a measure preserving flow acting on a standard Borel probability space $\left(X^{i}, \mathcal{B}^{i}, \mu^{i}\right)$. We say that a measure $\lambda$ on $\left(X^{1} \times \ldots \times X^{K}, \mathcal{B}^{1} \otimes \ldots \otimes \mathcal{B}^{K}\right)$ is a $K$ - joining if it is $\mathcal{T}^{1} \times \ldots \times \mathcal{T}^{K}$-invariant and it projects on $X^{i}$ as $\mu^{i}$ for each $i=1, \ldots, K$. We denote by $J\left(\mathcal{T}^{1}, \ldots, \mathcal{T}^{K}\right)$ the set of all joinings of $\mathcal{T}^{i}$ for $i=1, \ldots, K$ and by $J^{e}\left(\mathcal{T}^{1}, \ldots, \mathcal{T}^{K}\right)$ the subset of ergodic joinings. If for $i=1, \ldots, K$, $\left(X^{i}, \mathcal{B}^{i}, \mu^{i}, \mathcal{T}^{i}\right)$ are copies of the same flow, then we say that $\lambda$ is a $K$-self joining. We denote the set of $K$-self joinings of a flow $\mathcal{T}$ by $J_{K}(\mathcal{T})$ and ergodic $K$-self joinings by $J_{K}^{e}(\mathcal{T})$. If $\mathcal{T}^{1}, \ldots, \mathcal{T}^{K}$ are ergodic, then the following remarks hold.

Remark 2.1. $J\left(\mathcal{T}^{1}, \ldots, \mathcal{T}^{K}\right)$ is a compact simplex in the space of all $\mathcal{T}^{1} \times \ldots \times \mathcal{T}^{K}$-invariant measures and its set of extremal points $\left(J\left(\mathcal{T}^{1}, \ldots, \mathcal{T}^{K}\right)\right)$ equals $J^{e}\left(\mathcal{T}^{1}, \ldots, \mathcal{T}^{K}\right)$.

Remark 2.2 (Ergodic decomposition). For each $\lambda \in J\left(\mathcal{T}^{1}, \ldots, \mathcal{T}^{K}\right)$ there exists a unique probability measure $\kappa$ on $J^{e}\left(\mathcal{T}^{1}, \ldots, \mathcal{T}^{K}\right)$ such that

$$
\lambda=\int_{J^{e}\left(\mathcal{T}^{1}, \ldots, \mathcal{T}^{K}\right)} \rho d \kappa(\rho) .
$$

Assume that $K=2$. Note that $\mu^{1} \otimes \mu^{2} \in J\left(\mathcal{T}^{1}, \mathcal{T}^{2}\right)$. We say that the flows $\mathcal{T}^{1}$ and $\mathcal{T}^{2}$ are disjoint in the sense of Furstenberg (or simply disjoint) if the product measure is the only joining between them.

Remark 2.3. If two flows are disjoint, then they have no common factor. In particular, they are not isomorphic.

The notions of joinings and disjointness can be rewritten for automorphisms instead of flows. Then we also have the following well-known observation.

Remark 2.4. If $(X, \mathcal{B}, \mu, T)$ is an ergodic automorphism and $(Y, \mathcal{C}, \nu, I d)$ is the identity then $T$ and $I d$ are disjoint. 
Let $\phi:\left(X^{1}, \mathcal{B}^{1}, \mu^{1}, \mathcal{T}^{1}\right) \rightarrow\left(X^{2}, \mathcal{B}^{2}, \mu^{2}, \mathcal{T}^{2}\right)$ be an isomorphism. It is easy to see that $\mu_{\phi}^{1}:=$ $(\operatorname{Id} \times \phi)_{*} \mu^{1}$ is a joining of $\mathcal{T}^{1}$ and $\mathcal{T}^{2}$. We say that $\mu_{\phi}^{1}$ is a graph joining. We have the following remark.

Remark 2.5. Let $\lambda \in J\left(\mathcal{T}^{1}, \mathcal{T}^{2}\right)$ and let $\Pi \subseteq \mathcal{B}^{1}$ be a family of measurable sets. Let $\phi$ : $\left(X^{1}, \mathcal{B}^{1}, \mu^{1}, \mathcal{T}^{1}\right) \rightarrow\left(X^{2}, \mathcal{B}^{2}, \mu^{2}, \mathcal{T}^{2}\right)$ be an isomorphism. Then the following are equivalent:

(1) $\lambda(A \times B)=\mu^{1}\left(A \cap \phi^{-1}(B)\right)$ for all $A \in \Pi$ and $B \in \phi(\Pi)$;

(2) $\lambda(A \times X \triangle X \times \phi A)=0$ for every $A \in \Pi$;

(3) $\lambda\left(A \times \phi A^{c}\right)=\lambda\left(A^{c} \times \phi A\right)=0$ for every $A \in \Pi$.

Consider graph joinings between two identical flows $(X, \mathcal{B}, \mu, \mathcal{T})$. If $\phi=T_{-t}$ for some $t \in \mathbb{R}$ then we say that $\mu_{\phi}$ is a 2-off-diagonal joining and we denote it by $\mu_{t}$. In other words for $A, B \in \mathcal{B}$ we have

$$
\mu_{t}(A \times B)=\mu\left(A \cap T_{t} B\right)=\mu\left(T_{-t} A \cap B\right) .
$$

This definition is easily extended to higher dimensions, namely a $K$-off-diagonal joining $\mu_{t_{1}, \ldots, t_{K-1}}$ is a $K$-joining given by the formula

$$
\mu_{t_{1}, \ldots, t_{K-1}}\left(A_{1} \times \ldots \times A_{K}\right)=\mu\left(T_{-t_{1}} A_{1} \cap \ldots \cap T_{-t_{K-1}} A_{K-1} \cap A_{K}\right),
$$

for all $A_{1}, \ldots, A_{K} \in \mathcal{B}$.

Let $\mathcal{P}\left(\mathbb{R}^{K-1}\right)$ be the set of Borel probability measures on $\mathbb{R}^{K-1}$. For every $P \in \mathcal{P}\left(\mathbb{R}^{K-1}\right)$ we consider the $K$-integral joining given by

$$
\begin{aligned}
\left(\int_{\mathbb{R}^{K-1}} \mu_{t_{1}, \ldots, t_{K-1}} d P\left(t_{1}, \ldots, t_{K-1}\right)\right)\left(A_{1} \times \ldots \times A_{K}\right) \\
:=\int_{\mathbb{R}^{K-1}} \mu_{t_{1}, \ldots, t_{K-1}}\left(A_{1} \times \ldots \times A_{K}\right) d P\left(t_{1}, \ldots, t_{K-1}\right),
\end{aligned}
$$

where $A_{1}, \ldots, A_{K} \in \mathcal{B}$.

A Markov operator $\Psi: L^{2}(X, \mathcal{B}, \mu) \rightarrow L^{2}(X, \mathcal{B}, \mu)$ is a linear operator which satisfies

(1) it is a contraction, that is $\|\Psi f\|_{2} \leq\|f\|_{2}$ for every $f \in L^{2}(X, \mathcal{B}, \mu)$;

(2) $f \geq 0 \Rightarrow \Psi(f) \geq 0$

(3) $\Psi(\mathbf{1})=\mathbf{1}=\Psi^{*}(\mathbf{1})$.

With every 2-self joining $\lambda \in J_{2}(\mathcal{T})$, we can associate a Markov operator $\Psi(\lambda): L^{2}(X, \mathcal{B}, \mu) \rightarrow$ $L^{2}(X, \mathcal{B}, \mu)$ such that

$$
\lambda(A \times B)=\int_{X} \Psi(\lambda)\left(\chi_{A}\right) \chi_{B} d \mu \text { for any } A, B \in \mathcal{B} .
$$

Denote by $\mathcal{J}(\mathcal{T})$ the set of all Markov operators which commute with the Koopman operator associated with $\mathcal{T}$. It appears that if we consider weak-* topology on $\mathcal{J}(\mathcal{T})$, then $(2)$ defines an affine homeomorphism $\Psi: J_{2}(\mathcal{T}) \rightarrow \mathcal{J}(\mathcal{T})$. For more information about joinings and Markov operators we refer to [10].

Consider the affine continuous map $\Pi_{1,3}: J_{3}(\mathcal{T}) \rightarrow J_{2}(\mathcal{T})$ given by

$$
\Pi_{1,3}(\lambda)(A \times B):=\lambda(A \times X \times B) \text { for any } A, B \in \mathcal{B} .
$$

In other words $\Pi_{1,3}(\lambda)$ is the projection of the joining $\lambda$ on the first and third coordinates. Analogously, we define $\Pi_{2,3}$, the projection on the second and third coordinates. Since $J_{2}(\mathcal{T})$ and $\mathcal{J}(\mathcal{T})$ are affinely homeomorphic, we can consider the affine continuous maps $\Psi \circ \Pi_{i, 3}$ : $J_{3}(\mathcal{T}) \rightarrow \mathcal{J}(\mathcal{T})$ for $i=1,2$. Note that for any $t, s \in \mathbb{R}$ we have

$$
\Psi \circ \Pi_{1,3}\left(\mu_{t, s}\right)=T_{-t}, \text { and } \Psi \circ \Pi_{2,3}\left(\mu_{t, s}\right)=T_{-s} .
$$


For $i \in\{1,2\}$ let $\sigma_{i}: \mathbb{R}^{2} \rightarrow \mathbb{R}$ be the projection on the $i$-th coordinate. Then for every $P \in \mathcal{P}\left(\mathbb{R}^{2}\right)$, we also have

$$
\begin{aligned}
\Pi_{i, 3}\left(\int_{\mathbb{R}^{2}} \mu_{-t,-s} d P(t, s)\right) & =\int_{\mathbb{R}^{2}} \Pi_{i, 3}\left(\mu_{-t,-s}\right) d P(t, s) \text { and } \\
\Psi \circ \Pi_{i, 3}\left(\int_{\mathbb{R}^{2}} \mu_{-t,-s} d P(t, s)\right) & =\int_{\mathbb{R}} T_{t} d\left(\left(\sigma_{i}\right)_{*} P\right)(t),
\end{aligned}
$$

for $i=1,2$.

2.2. Special flows. Let $(X, \mathcal{B}, \mu)$ be a standard Borel probability space. Let $T: X \rightarrow X$ be an ergodic $\mu$-preserving automorphism. Let $f \in L^{1}([0,1))$ be positive and for any $n \in \mathbb{Z}$ consider

$$
f^{(n)}(x):= \begin{cases}\sum_{i=0}^{n-1} f\left(T^{i} x\right) & \text { if } n \geq 1 \\ 0 & \text { if } n=0 \\ -\sum_{i=n}^{-1} f\left(T^{i} x\right) & \text { if } n \leq-1 .\end{cases}
$$

Define $X^{f}:=((x, r) ; x \in X, 0 \leq r<f(x))$ and on $X^{f}$ consider the measure $\mu \otimes \operatorname{Leb}\left(\left.\right|_{X^{f}}\right.$. The special flow $T^{f}=\left\{T_{t}^{f}\right\}_{t \in \mathbb{R}}$ is the measure preserving flow acting on $X^{f}$ by the formula

$$
T_{t}^{f}(x, r):=\left(T^{n} x, r+t-f^{(n)}(x)\right),
$$

where $n \in \mathbb{Z}$ is unique, such that $f^{(n)}(x) \leq r+t<f^{(n+1)}(x)$. We say that $f$ is the roof function and $T$ is the base of the special flow. In view of Ambrose Representation Theorem (see [1]), every ergodic flow is measure theoretically isomorphic to a special flow. Such a special flow is called a special representation of the flow. In this paper we deal with special flows whose roof functions are piecewise continuous and whose bases are interval exchange transformations. We always assume that roof functions are right-continuous and that the left limits exist. If a piecewise continuous bounded function $f$ has a discontinuity at $x$, then the jump at $x$ is the number $d:=f(x)-\lim _{y \rightarrow x^{-}} f(x)$.

2.3. Space of flows. Let $(X, \mathcal{B}(X), \mu)$ be a standard Borel probability space. By Flow $(X)$ we denote the set of all measure preserving flows on $X$. Let $\mathcal{T}=\left\{T_{t}\right\}_{t \in \mathbb{R}} \in$ Flow $(X), A \in \mathcal{B}(X)$ and $\varepsilon>0$. Let

$$
U(\mathcal{T}, A, \varepsilon):=\left\{\mathcal{S}=\left\{S_{t}\right\}_{t \in \mathbb{R}} \in \operatorname{Flow}(X) ; \sup _{t \in[-1,1]} \mu\left(T_{t} A \triangle S_{t} A\right)<\varepsilon\right\} .
$$

It appears that the family of sets of the above form gives a subbase of a topology, and Flow $(X)$ endowed with this topology is a Polish space.

Let $(Y, d)$ be a metric space. It follows that a map $F: Y \rightarrow \operatorname{Flow}(X)$ is continuous if for any $y \in Y$ and $A \in \mathcal{B}(X)$ we have

(6) for any $\varepsilon>0$ there exists $\delta>0$ such that $d(y, z)<\delta \Rightarrow F(z) \in U(F(y), A, \varepsilon)$.

By using the fact that for any $A_{1}, B_{1}, A_{2}, B_{2} \in \mathcal{B}(X)$ we have

$$
A_{1} \triangle B_{1}=A_{1}^{c} \triangle B_{1}^{c} \text { and }\left(A_{1} \cup B_{1}\right) \triangle\left(A_{2} \cup B_{2}\right) \subseteq\left(A_{1} \triangle A_{2}\right) \cup\left(B_{1} \triangle B_{2}\right),
$$

we can prove that the set of all $A \in \mathcal{B}(X)$, for which for every $\varepsilon>0$ there exists $\delta_{A}$ such that (6) is satisfied, form an algebra. By using the triangle inequality

$$
\mu(A \triangle B) \leq \mu(A \triangle C)+\mu(B \triangle C) \text { for } A, B, C \in \mathcal{B}(X),
$$

we can prove that this algebra is closed under taking the countable union of increasing family of sets and thus, it is a $\sigma$-algebra. Hence it is enough to check (6) for a family of sets which generates $\mathcal{B}(X)$.

All non-atomic standard Borel probability spaces are measure theoretically isomorphic (see Theorem 3.4.23 in [21]). Let $\left(X_{1}, \mathcal{B}\left(X_{1}\right), \mu_{1}\right)$ and $\left(X_{2}, \mathcal{B}\left(X_{2}\right), \mu_{2}\right)$ be standard Borel non-atomic 
probability spaces and let $H: X_{1} \rightarrow X_{2}$ be some isomorphism. Then Flow $\left(X_{1}\right)$ and Flow $\left(X_{2}\right)$ can be identified by a homeomorphism $\phi: \operatorname{Flow}\left(X_{1}\right) \rightarrow \operatorname{Flow}\left(X_{2}\right)$ given by the formula

$$
\phi(\mathcal{T}):=H \circ \mathcal{T} \circ H^{-1} \text {. }
$$

Remark 2.6. To prove that $F:(Y, d) \rightarrow$ Flow $\left(X_{1}\right)$ is continuous, we can instead prove that $\phi \circ F:(Y, d) \rightarrow$ Flow $\left(X_{2}\right)$ is continuous. In other words, we need to prove that for every $y \in Y$ and $A \in \mathcal{D} \subset \mathcal{B}\left(X_{2}\right)$, where $\mathcal{D}$ generates $\mathcal{B}\left(X_{2}\right)$, we have

for any $\varepsilon>0$ there exists $\delta>0$ such that $d(y, z)<\delta \Rightarrow \phi \circ F(z) \in U(\phi \circ F(y), A, \varepsilon)$.

2.4. Interval exchange transformations. Let $\mathcal{A}$ be an alphabet of $d$ elements. Let now $\epsilon \in\{0,1\}$ and let $\pi_{\epsilon}: \mathcal{A} \rightarrow\{1, \ldots, d\}$ be bijections. We will now consider a permutation $\pi$ as a pair $\left\{\pi_{0}, \pi_{1}\right\}$ where $\pi_{0}(\alpha)$ corresponds to the position of letter $\alpha$ before permutation, while $\pi_{1}(\alpha)$ defines the position of $\alpha$ after permutation. We say that a permutation $\pi$ is irreducible if there is no $1 \leq k<d$ such that

$$
\pi_{1} \circ \pi_{0}^{-1}(\{1, \ldots, k\})=\{1, \ldots, k\} .
$$

In this paper we will only deal with irreducible permutations, so this assumption will usually be omitted. We say that the permutation is symmetric if

$$
\pi_{1}(\alpha)=d+1-\pi_{0}(\alpha) \text { for every } \alpha \in \mathcal{A} .
$$

Note that a symmetric permutation is always irreducible.

The intervals that we will now consider are always left-side closed and right-side open unless told otherwise. Let $I$ be an interval equipped with its Borel $\sigma$-algebra and Lebesgue measure Leb(. Without losing generality, we can assume that the left endpoint of $I$ is 0 . Let $\left\{I_{\alpha}\right\}_{\alpha \in \mathcal{A}}$ be a partition of $I$ into $d$ intervals, where $I_{\alpha}$ has length $\lambda_{\alpha} \geq 0$. We will denote $\lambda:=\left\{\lambda_{\alpha}\right\}_{\alpha \in \mathcal{A}}$ the length vector and obviously we have $|\lambda|:=\sum_{\alpha \in \mathcal{A}} \lambda=\operatorname{Leb}(I)$. The interval exchange transformation (IET) $T_{\pi, \lambda}: I \rightarrow I$ is the automorphism which permutes intervals $I_{\alpha}$ according to the permutation $\pi$. Let now $\Omega_{\pi}:=\left[\left(\Omega_{\pi}\right)_{\alpha \beta}\right]_{\alpha, \beta \in \mathcal{A}}$ be the $d \times d$ matrix given by the following formula

$$
\left(\Omega_{\pi}\right)_{\alpha \beta}:= \begin{cases}+1 & \text { if } \pi_{0}(\alpha)<\pi_{0}(\beta) \text { and } \pi_{1}(\alpha)>\pi_{1}(\beta) \\ -1 & \text { if } \pi_{0}(\alpha)>\pi_{0}(\beta) \text { and } \pi_{1}(\alpha)<\pi_{1}(\beta) \\ 0 & \text { otherwise. }\end{cases}
$$

We will say that $\Omega_{\pi}$ is the translation matrix of $T^{\pi, \lambda}$. The name of the matrix is derived from the fact that $T_{\pi, \lambda}$ acts on an interval $I_{\alpha}$ as a translation by number $\sum_{\beta \in \mathcal{A}}\left(\Omega_{\pi}\right)_{\alpha \beta} \lambda_{\beta}$.

Let $\partial I_{\alpha}$ be the left endpoint of $I_{\alpha}$. We say that the IET $T_{\pi, \lambda}$ satisfies Keane's condition if

$$
T_{\pi, \lambda}^{m}\left(\partial I_{\alpha}\right)=\partial I_{\beta} \text { for } m>0 \text { implies } \alpha=\pi_{1}^{-1}(1), \beta=\pi_{0}^{-1}(1) \text { and } m=1 .
$$

It is easy to see that $T_{\pi, \lambda}$ satisfies Keane's condition whenever $\lambda$ is a rationally independent vector (that is there is no nontrivial integer linear combination of numbers $\lambda_{\alpha}$, which will give a rational number).

Denote by $S_{\mathcal{A}}^{0}$ the set of all irreducible permutations of $\mathcal{A}$. We may consider the space $S_{\mathcal{A}}^{0} \times \mathbb{R}_{\geq 0}^{\mathcal{A}}$ of all IETs of $d$ intervals. Define the operator $R: S_{\mathcal{A}}^{0} \times \mathbb{R}_{>0}^{\mathcal{A}} \rightarrow S_{\mathcal{A}}^{0} \times \mathbb{R}_{\geq 0}^{\mathcal{A}}$, such that $R(\pi, \lambda)=R\left(T_{\pi, \lambda}\right)$ is the first return map of $T_{\pi, \lambda}$ to the interval $\left[0,|\lambda|-\min \left\{\lambda_{\pi_{0}^{-1}(d)}, \lambda_{\pi_{1}^{-1}(d)}\right\}\right)$. The operator $R$ is called the Rauzy-Veech induction (or righthand side Rauzy-Veech induction). The Rauzy classes are the minimal subsets of $S_{\mathcal{A}}^{0}$ which are invariant under the induced action of $R$ on $S_{\mathcal{A}}^{0}$.

Let

$$
l:\{1, \ldots, d\} \rightarrow\{1, \ldots, d\} \text { be given by } l(i)=d+1-i .
$$

The function $l$ acts on $S_{\mathcal{A}}^{0}$ by mapping $\left\{\pi_{0}, \pi_{1}\right\}$ onto $\left\{l \circ \pi_{0}, l \circ \pi_{1}\right\}$. The extended Rauzy classes are minimal subsets of $S_{\mathcal{A}}^{0}$ which are invariant under $R$ and action of $l$. We have the following result. 
Theorem 2.7 (Rauzy). Any Rauzy class of permutations of $d \geq 2$ elements contains at least one permutation $\pi$ such that

$$
\pi_{1} \circ \pi_{0}^{-1}(1)=d \quad \text { and } \quad \pi_{1} \circ \pi_{0}^{-1}(d)=1 .
$$

2.5. Translation surfaces and moduli space. Let $M$ be an orientable compact and connected topological surface of genus $g \geq 1$. Let $\Sigma:=\left\{A_{1}, \ldots, A_{s}\right\}$ be a finite subset of singular points in $M$. Let $\kappa:=\left(\kappa_{1}, \ldots, \kappa_{s}\right)$ be a vector of positive integers satisfying $\sum_{i=1}^{s} \kappa_{i}=2 g-2$. A translation structure on $M$ is a maximal atlas $\zeta$ on $M \backslash \Sigma$ of charts by open sets of $\mathbb{C}$ such that any coordinate change between charts is a translation and for each $1 \leq i \leq s$ there exists a neighbourhood $V_{i} \subset M$ of $A_{i}$, a neigbourhood $W_{i} \subset \mathbb{C}$ of 0 and a ramified covering $\pi:\left(V_{i}, A_{i}\right) \rightarrow\left(W_{i}, 0\right)$ of degree $\kappa_{i}+1$ such that each injective restriction of $\pi$ is a chart of $\zeta$. On $(M, \zeta)$ we can consider a holomorphic 1 -form which in the local coordinates can be written as $d z$. We will denote this form also by $\zeta$. We identify the associated 2 -form $\frac{i}{2} \zeta \wedge \bar{\zeta}$ with the Lebesgue measure $\lambda_{\zeta}$ on $M$. Moreover, the quadratic form $|\zeta|^{2}$ yields a Riemannian metric $(M, \zeta)$. By $d_{\zeta}$ we denote the distance given by this metric. We also consider on $(M, \zeta)$ a vertical translation flow, denoted by $\mathcal{T}^{\zeta}=\left\{\mathcal{T}_{t}^{\zeta}\right\}_{t \in \mathbb{R}}$, which in local coordinates is a unit speed flow in the vertical direction. The flow $\mathcal{T}^{\zeta}$ preserves $\lambda_{\zeta}$ and thus can be viewed as an element of $\operatorname{Flow}\left(M, \lambda_{\zeta}\right)$.

In the set of all translation structures on $M$ we identify the structures $\zeta_{1}$ and $\zeta_{2}$ if there exists a homeomorphism $H: M \rightarrow M$ which sends singular points of $\zeta_{1}$ onto singular points of $\zeta_{2}$ and $\zeta_{1}=H^{*} \zeta_{2}$. In terms of local coordinates, $H$ is locally a translation. This is an equivalence relation and its equivalence classes form the moduli space denoted by $\operatorname{Mod}(M)$. The moduli space can be divided into subsets called strata $\mathcal{M}(M, \Sigma, \kappa)=\mathcal{M}(\kappa)$, for which the vector of degrees of singularities is given by $\kappa$. It appears that each such stratum $\mathcal{M}(M, \Sigma, \kappa)$ is a complex orbifold (see [22]) and has a finite number of connected components (see [17]). On $\mathcal{M}$ we can consider an action of $S L(2, \mathbb{R})$. It is given by composing the charts of a translation surface with a given linear map. The strata are invariant under the action of $S L(2, \mathbb{R})$. It is worth noting that in particular for every $\theta \in \mathbb{R} / \mathbb{Z}$ we can apply the rotation $r_{\theta}$ by $\theta$ to the translation structure and almost every angle $\theta$ yields no saddle connection of a vertical flow.

Let $\pi=\left(\pi_{0}, \pi_{1}\right)$ be a permutation of the alphabet $\mathcal{A}$ of $d>1$ elements and let $\lambda \in \mathbb{R}_{\geq 0}^{\mathcal{A}}$. Consider also $\tau \in \mathbb{R}^{\mathcal{A}}$ such that for each $1 \leq k<d$ we have

$$
\sum_{\left\{\alpha ; \pi_{0}(\alpha) \leq k\right\}} \tau_{\alpha}>0 \text { and } \sum_{\left\{\alpha ; \pi_{1}(\alpha) \leq k\right\}} \tau_{\alpha}<0 .
$$

Moreover we require that

$$
\pi_{i}(\alpha)=\pi_{i}(\beta)+1 \wedge \lambda_{\alpha}=\lambda_{\beta}=0 \Rightarrow \tau_{\alpha} \cdot \tau_{\beta}>0 \text { for all } i=0,1 \text { and } \alpha, \beta \in \mathcal{A} .
$$

For a fixed permutation $\pi$, we denote by $\Theta_{\pi}$ the set of triples $(\pi, \lambda, \tau)$ satisfying the above conditions.

Consider the polygonal curve in $\mathbb{C}$ obtained by connecting the points 0 and $\sum_{i \leq k}\left(\lambda_{\pi_{0}^{-1}(i)}+\right.$ $\left.i \tau_{\pi_{0}^{-1}(i)}\right)$ for $k=1, \ldots, d$, using the line segments. Analogously we can consider the polygonal curve obtained by connecting the points 0 and $\sum_{i \leq k}\left(\lambda_{\pi_{1}^{-1}(i)}+i \tau_{\pi_{1}^{-1}(i)}\right)$ for $k=1, \ldots, d$. These two polygonal curves define a polygon with $d$ pairs of parallel sides. By identifying those sides we obtain a translation surface $M$, with $\Sigma$ being the set of vertices of the polygon (some of them may be identified). We denote by $M(\pi, \lambda, \tau)$ the translation structure given by $(\pi, \lambda, \tau)$.

It appears that whenever $\mathcal{T}^{\zeta}$ admits no saddle-connections, $\zeta$ can be viewed as $M(\pi, \lambda, \tau)$ for some $(\pi, \lambda, \tau) \in \Theta_{\pi}$, with $\pi$ being some permutation (see e.g. [23]). Moreover we can consider $(\pi, \lambda, \tau) \in \Theta_{\pi}$ as local coordinates in the neighbourhood of such $\zeta$ in the corresponding stratum. Since almost every rotation yields no saddle-connections, to obtain local coordinates in the neighbourhood of $\zeta$ for which $\mathcal{T}^{\zeta}$ has a saddle connection, we can use the rotation to obtain local coordinates around rotated form and then rotate it back.

Kontsevich and Zorich in [17, gave a complete characterization of connected components of strata in the moduli space. In particular, they showed that each stratum $\mathcal{M}(2 g-2)$ and 
$\mathcal{M}(g-1, g-1)$, where $g$ is the genus of the surface, contains exactly one so-called hyperelliptic connected component, which we denote by $\mathcal{M}^{\text {hyp }}(2 g-2)$ and $\mathcal{M}^{\text {hyp }}(g-1, g-1)$ respectively. For every hyperelliptic component $C \subset \mathcal{M}$, there exists an involution $\phi: M \rightarrow M$ such that for every $\zeta \in C$ we have $\phi^{*} \zeta=-\zeta$. In particular we have the following remark.

Remark 2.8. For every hyperelliptic connected component $C \subset \mathcal{M}$ and for every $\zeta \in G$, the vertical flow on $(M, \zeta)$ is isomorphic with its inverse.

It appears that the connected components of the moduli space can be described by the Rauzy classes of permutations. Let us recall first the notion of non-degenericity, as introduced by Veech. We say that a permutation $\pi=\left\{\pi_{0}, \pi_{1}\right\}$ of $\mathcal{A}$ is degenerate if one of the following conditions is satisfied:

$$
\begin{aligned}
& \pi_{1} \circ \pi_{0}^{-1}(j+1)=\pi_{1} \circ \pi_{0}^{-1}(j)+1 \text { for some } 1 \leq j<d ; \\
& \pi_{1} \circ \pi_{0}^{-1}\left(\pi_{0} \circ \pi_{1}^{-1}(d)+1\right)=\pi_{1} \circ \pi_{0}^{-1}(d)+1 \\
& \pi_{0} \circ \pi_{1}^{-1}(1)-1=\pi_{0} \circ \pi_{1}^{-1}\left(\pi_{1} \circ \pi_{0}^{-1}(1)-1\right) \\
& \pi_{0} \circ \pi_{1}^{-1}(d)=\pi_{0} \circ \pi_{1}^{-1}(1)-1 \text { and } \pi_{1} \circ \pi_{0}^{-1}(d)=\pi_{1} \circ \pi_{0}^{-1}(1)-1 .
\end{aligned}
$$

Otherwise the permutation is called non-degenerate. The property of non-degenericity is invariant under the action of the Rauzy-Veech induction. The importance of this notion is given by the following theorem.

Theorem 2.9 (Veech). The extended Rauzy classes of nondegenerate permutations are in oneto-one correspondence with the connected components of the strata in the moduli space.

In view of the above theorem, for each genus $g \geq 2$, the hyperelliptic components $\mathcal{M}^{\text {hyp }}(2 g-$ 2 ) and $\mathcal{M}^{\text {hyp }}(g-1, g-1)$ correspond to the extended Rauzy classes of symmetric permutations of $2 g$ and $2 g-1$ elements respectively.

Remark 2.10. Connected components which are associated with extended Rauzy graphs of permutations of $d \leq 5$ elements are hyperelliptic.

For a given extended Rauzy class $\mathcal{R}$, let $C_{\mathcal{R}}$ be its associated connected component of the moduli space. Then for any $\pi \in \mathcal{R}$ the map $M: \Theta_{\pi} \rightarrow C_{\mathcal{R}}$ given by $(\pi, \lambda, \tau) \mapsto M(\pi, \lambda, \tau)$ is continuous and the range of the map $M$ is dense in $C_{\mathcal{R}}$. Moreover, recall that, due to Theorem 2.7. for every connected component of the moduli space we can find a permutation $\bar{\pi}$ belonging to the corresponding extended Rauzy class, satisfying $\bar{\pi}_{1} \circ \bar{\pi}_{0}^{-1}(d)=1$ and $\bar{\pi}_{0} \circ \bar{\pi}_{1}^{-1}(d)=1$. Hence, to prove that some condition is satisfied for a dense set of translation structures in $C_{\mathcal{R}}$, it is enough to prove that it holds for translation structures, whose associated polygonal parameters belong to a dense subset of $\Theta_{\bar{\pi}}$.

Let $\mathcal{R}$ be any extended Rauzy class. Let us consider a transformation $\tilde{R}: \bigcup_{\pi \in \mathcal{R}} \Theta_{\pi} \mapsto$ $\bigcup_{\pi \in \mathcal{R}} \Theta_{\pi}$ called a polygonal Rauzy Veech induction (or righthand side polygonal Rauzy Veech induction) which yields different parameters of a translation surface.

Let $\pi \in \mathcal{R}$ and let $(\pi, \lambda, \tau) \in \Theta_{\pi}$. Assume that $\lambda_{\pi_{0}^{-1}(d)} \neq \lambda_{\pi_{1}^{-1}(d)}$. If $\lambda_{\pi_{0}^{-1}(d)}<\lambda_{\pi_{1}^{-1}(d)}$, then for any $a \in \mathcal{A}$ define

$$
\begin{aligned}
\tilde{\pi}_{0}(a) & :=\left\{\begin{array}{lll}
\pi_{0}(a) & \text { if } \quad \pi_{0}(a) \leq \pi_{0}\left(\pi_{1}^{-1}(d)\right) ; \\
\pi_{0}\left(\pi_{1}^{-1}(d)\right)+1 & \text { if } \quad \pi_{0}(a)=d ; \\
\pi_{0}(a)+1 & \text { if } & \pi_{0}\left(\pi_{1}^{-1}(d)\right)<\pi_{0}(a) \leq d-1,
\end{array}\right. \\
\tilde{\pi}_{1}(a) & :=\pi_{1}(a), \\
\tilde{\lambda}_{a} & := \begin{cases}\lambda_{\pi_{1}^{-1}(d)}-\lambda_{\pi_{0}^{-1}(d)} & \text { if } \pi_{1}(a)=d ; \\
\lambda_{a} & \text { otherwise, }\end{cases} \\
\tilde{\tau}_{a} & := \begin{cases}\tau_{\pi_{1}^{-1}(d)}-\tau_{\pi_{0}^{-1}(d)} & \text { if } \pi_{1}(a)=d ; \\
\tau_{a} & \text { otherwise. }\end{cases}
\end{aligned}
$$


Analogously, if $\lambda_{\pi_{0}^{-1}(d)}>\lambda_{\pi_{1}^{-1}(d)}$, we define

$$
\begin{aligned}
\tilde{\pi}_{0}(a) & :=\pi_{0}(a), \\
\tilde{\pi}_{1}(a) & :=\left\{\begin{array}{lll}
\pi_{1}(a) & \text { if } & \pi_{1}(a) \leq \pi_{1}\left(\pi_{0}^{-1}(d)\right) ; \\
\pi_{1}\left(\pi_{0}^{-1}(d)\right)+1 & \text { if } & \pi_{1}(a)=d ; \\
\pi_{1}(a)+1 & \text { if } & \pi_{1}\left(\pi_{0}^{-1}(d)\right)<\pi_{1}(a) \leq d-1,
\end{array}\right. \\
\tilde{\lambda}_{a} & :=\left\{\begin{array}{lll}
\lambda_{\pi_{0}^{-1}(d)}-\lambda_{\pi_{1}^{-1}(d)} & \text { if } & \pi_{0}(a)=d ; \\
\lambda_{a} & \text { otherwise, }
\end{array}\right. \\
\tilde{\tau}_{a} & := \begin{cases}\tau_{\pi_{0}^{-1}(d)}-\tau_{\pi_{1}^{-1}(d)} & \text { if } \pi_{0}(a)=d ; \\
\tau_{a} & \text { otherwise. }\end{cases}
\end{aligned}
$$

We define $\tilde{R}$ by setting $\tilde{R}(\pi, \lambda, \tau):=(\tilde{\pi}, \tilde{\lambda}, \tilde{\tau})$. It is defined almost everywhere on $\bigcup_{\pi \in \mathcal{R}} \Theta_{\pi}$ and if $M(\pi, \lambda, \tau)$ admits no saddle connection, it can be iterated indefinitely. Similarly, we can also define a left hand side polygonal Rauzy Veech induction. Note that the polygons derived from $(\pi, \lambda, \tau)$ and $(\tilde{\pi}, \tilde{\lambda}, \tilde{\tau})$ represent the same translation surface, i.e. $M(\pi, \lambda, \tau)=M(\tilde{\pi}, \tilde{\lambda}, \tilde{\tau})$. Indeed, the latter is obtained from $(\pi, \lambda, \tau)$ by cutting out the triangle formed by the last top side and the last bottom side and gluing it to a side of a polygon which is identified with one of the two sides forming the triangle.

Every $\zeta \in C_{\pi}$ which does not have vertical saddle-connections can be represented as $M(\pi, \lambda, \tau)$, for some $(\pi, \lambda, \tau) \in \Theta_{\pi}$. We can consider the metric on the neighbourhood of $M(\pi, \lambda, \tau)$ on $\mathcal{M}(M, \Sigma, \kappa)$ given by

$$
d_{M o d}\left(\left(\pi, \lambda^{\prime}, \tau^{\prime}\right),\left(\pi, \lambda^{\prime \prime}, \tau^{\prime \prime}\right)\right):=\sum_{a \in \mathcal{A}}\left(\left|\lambda_{a}^{\prime}-\lambda_{a}^{\prime \prime}\right|+\left|\tau_{a}^{\prime}-\tau_{a}^{\prime \prime}\right|\right) .
$$

If $\zeta$ admits vertical saddle-connections, we can apply $r_{\theta}$ for some $\theta \in \mathbb{R} / \mathbb{Z}$, so that $r_{\theta}^{*} \zeta$ does not have vertical saddle-connections and then define a metric in the neighbourhood of $\zeta$.

For any $\zeta=M(\pi, \lambda, \tau) \in \mathcal{M}(M, \Sigma, \kappa)$ we can consider a special representation of the vertical flow on $(M, \zeta)$. The basis of this special flow is the IET $T_{\pi, \lambda}$ and the roof function $h$ is positive and constant over exchanged intervals. Hence $h$ can be considered as a vector $\left(h_{a}\right)_{a \in \mathcal{A}} \in \mathbb{R}_{>0}^{\mathcal{A}}$, where $h_{a}$ is the value of $h$ over the exchanged interval labelled by $a$. The vector $h$ is given by the formula

$$
h=-\Omega_{\pi} \tau,
$$

where $\Omega_{\pi}$ is the translation matrix of $(\pi, \lambda)$. This gives rise to new local coordinates of the moduli space. In particular, the polygonal Rauzy-Veech induction receives a new form. Namely, if $\lambda_{\pi_{0}^{-1}(d)} \neq \lambda_{\pi_{1}^{-1}(d)}$ then $\tilde{R}(\pi, \lambda, h)=(\tilde{\pi}, \tilde{\lambda}, \tilde{h})$, where the formulas for $\tilde{\pi}$ and $\tilde{\lambda}$ remain unchanged and for any $a \in \mathcal{A}$ we take

$$
\tilde{h}_{a}:= \begin{cases}h_{\pi_{0}^{-1}(d)}+h_{\pi_{1}^{-1}(d)} & \text { if } a=\pi_{0}^{-1}(d) \text { and } \lambda_{\pi_{0}^{-1}(d)}<\lambda_{\pi_{1}^{-1}(d)} \\ h_{\pi_{0}^{-1}(d)}+h_{\pi_{1}^{-1}(d)} & \text { if } a=\pi_{1}^{-1}(d) \text { and } \lambda_{\pi_{0}^{-1}(d)}>\lambda_{\pi_{1}^{-1}(d)} \\ h_{a} & \text { otherwise }\end{cases}
$$

\section{Consequences OF Limit JOININGS}

In this section, we formulate a criterion for two flows to be disjoint, and a criterion for a flow to be weakly mixing. Both criteria rely on the properties of the weak limit of some sequence of 3-off diagonal joinings.

For every measure $\lambda \in \mathcal{P}(X \times Y)$, we denote by $\left.\lambda\right|_{X}$ and $\left.\lambda\right|_{Y}$ the projections of $\lambda$ on $X$ and $Y$ respectively, that is for every measurable subsets $A \subseteq X$ and $B \subseteq Y$ we have

$$
\left.\lambda\right|_{X}(A)=\lambda(A \times Y) \text { and }\left.\lambda\right|_{Y}(B)=\lambda(X \times B) .
$$


Let $\mathcal{T}=\left\{T_{t}\right\}_{t \in \mathbb{R}}$ and $\mathcal{S}=\left\{S_{t}\right\}_{t \in \mathbb{R}}$ be weakly mixing flows acting on standard Borel spaces $(X, \mathcal{B}, \mu)$ and $(Y, \mathcal{C}, \nu)$ respectively.

Lemma 3.1. Let $\lambda \in J^{e}(\mathcal{T}, \mathcal{S})$. Let $\rho \in J_{2}^{e}(\mathcal{T} \times \mathcal{S}, \lambda)$, which is defined on $X_{1} \times Y_{1} \times X_{2} \times Y_{2}$ with $X_{1}=X_{2}=X$ and $Y_{1}=Y_{2}=Y$. Assume that for some $r, r^{\prime} \in \mathbb{R}$ we have $\left.\rho\right|_{X_{1} \times X_{2}}=\mu_{T_{r}}$ and $\left.\rho\right|_{Y_{1} \times Y_{2}}=\nu_{S_{r^{\prime}}}$. If $r \neq r^{\prime}$ then $\lambda=\mu \otimes \nu$.

Proof. First we prove that $\lambda=\left(T_{r} \times S_{r^{\prime}}\right)_{*} \lambda$. We show that (3) in Remark 2.5 is satisfied for the $\pi$-system of product sets and the isomorphism $\phi:=T_{-r} \times S_{-r^{\prime}}$ between $\left(X_{1} \times Y_{1}, \lambda\right)$ and $\left(X_{2} \times Y_{2}, \lambda\right)$. In other words, for every $A \in \mathcal{B}$ and $B \in \mathcal{C}$ we have

$$
\rho\left(A \times B \times\left(T_{-r} \times S_{-r^{\prime}}\right)(A \times B)^{c}\right)=\rho\left((A \times B)^{c} \times\left(T_{-r} \times S_{-r^{\prime}}\right)(A \times B)\right)=0 .
$$

Indeed, recall that $\mu_{r}$ and $\nu_{r^{\prime}}$ are graph joinings of $\mathcal{T}$ and $\mathcal{S}$ given by $T_{-r}$ and $S_{-r^{\prime}}$ respectively. By Remark 2.5 this implies that for every $A \in \mathcal{B}$ and $B \in \mathcal{C}$ we have

$$
\mu_{r}\left(A \times T_{-r} A^{c}\right)=0 \quad \text { and } \quad \nu_{r^{\prime}}\left(B \times T_{-r} B^{c}\right)=0 .
$$

Thus we obtain

$$
\begin{aligned}
\rho(A \times B & \left.\times\left(T_{-r} \times S_{-r^{\prime}}\right)(A \times B)^{c}\right)=\rho\left(A \times B \times T_{-r} A^{c} \times S_{-r^{\prime}} B\right) \\
& +\rho\left(A \times B \times T_{-r} A^{c} \times S_{-r^{\prime}} B^{c}\right)+\rho\left(A \times B \times T_{-r} A \times S_{-r^{\prime}} B^{c}\right) \\
& \leq 2 \rho\left(A \times Y \times T_{-r} A^{c} \times Y\right)+\rho\left(X \times B \times X \times S_{-r^{\prime}} B^{c}\right) \\
& =2 \mu_{r}\left(A \times T_{-r} A^{c}\right)+\nu_{r^{\prime}}\left(B \times S_{-r^{\prime}} B^{c}\right)=0
\end{aligned}
$$

and

$$
\begin{aligned}
\rho\left((A \times B)^{c}\right. & \left.\times T_{-r} A \times S_{-r^{\prime}} B\right)=\rho\left(A^{c} \times B \times T_{-r} A \times S_{-r^{\prime}} B\right) \\
& +\rho\left(A^{c} \times B^{c} \times T_{-r} A \times S_{-r^{\prime}} B\right)+\rho\left(A \times B^{c} \times T_{-r} A \times S_{-r^{\prime}} B\right) \\
& \leq 2 \rho\left(A^{c} \times Y \times T_{-r} A \times Y\right)+\rho\left(X \times B^{c} \times X \times S_{-r^{\prime}} B\right) \\
& =2 \mu_{r}\left(A^{c} \times T_{-r} A\right)+\nu_{r^{\prime}}\left(B^{c} \times S_{-r^{\prime}} B\right)=0 .
\end{aligned}
$$

Hence we have proved that (3) in Remark 2.5 is satisfied for the $\pi$-system of product sets. Since $\rho \in J_{2}^{e}(\mathcal{T} \times \mathcal{S}, \lambda)$, in view of $(2)$ in Remark 2.5 we get

$$
\begin{aligned}
\lambda(A \times B) & =\rho(A \times B \times X \times Y)=\rho\left(X \times Y \times T_{-r} A \times S_{-r^{\prime}} B\right) \\
& =\lambda\left(T_{-r} A \times S_{-r^{\prime}} B\right)=\left(T_{r} \times S_{r^{\prime}}\right)_{*} \lambda(A \times B),
\end{aligned}
$$

for all $A \in \mathcal{B}$ and $B \in \mathcal{C}$. Since the $\pi$-system of product sets generates $\mathcal{B} \otimes \mathcal{C}$, we get that the measures $\lambda$ and $\left(T_{r} \times S_{r^{\prime}}\right)_{*} \lambda$ are equal. By the $(\mathcal{T} \times \mathcal{S})$-invariance of $\lambda$, we have that $\lambda$ is $\left(I d \times S_{r-r^{\prime}}\right)$-invariant. By weak mixing of $\mathcal{S}, S_{r-r^{\prime}}$ is ergodic whenever $r \neq r^{\prime}$. Since $I d$ is disjoint with every ergodic transformation (see Remark 2.4), we get $\lambda=\mu \otimes \nu$.

Proposition 3.2. Assume that for some real sequences $\left(a_{n}\right)_{n \in \mathbb{N}}$ and $\left(b_{n}\right)_{n \in \mathbb{N}}$ we have

$$
\mu_{a_{n}, b_{n}} \rightarrow(1-\alpha) \int_{\mathbb{R}^{2}} \mu_{-t,-u} d P(t, u)+\alpha \xi_{1},
$$

and

$$
\nu_{a_{n}, b_{n}} \rightarrow\left(1-\alpha^{\prime}\right) \int_{\mathbb{R}^{2}} \nu_{-t,-u} d Q(t, u)+\alpha^{\prime} \xi_{2},
$$

for some $0 \leq \alpha, \alpha^{\prime}<1$, measures $P, Q \in \mathcal{P}\left(\mathbb{R}^{2}\right)$ and $\xi_{1} \in J_{3}(\mathcal{T}), \xi_{2} \in J_{3}(\mathcal{S})$. Assume moreover, that there exists a set $B \in \mathcal{B}\left(\mathbb{R}^{2}\right)$, such that

$$
(1-\alpha) P(B)-\left(1-\alpha^{\prime}\right) Q(B)>\alpha^{\prime} .
$$

Then $\mathcal{T}$ and $\mathcal{S}$ are disjoint.

Remark 3.3. The above proposition can be also proven in higher dimensional case, that is when we consider limits of joinings of higher rank. 
Proof of Proposition 3.2. Let $\xi_{1}=\int_{J_{3}^{e}(\mathcal{T})} \rho^{\mathcal{T}} d \kappa_{1}\left(\rho^{\mathcal{T}}\right)$ and $\xi_{2}=\int_{J_{3}^{e}(\mathcal{S})} \rho^{\mathcal{S}} d \kappa_{2}\left(\rho^{\mathcal{S}}\right)$ be the ergodic decompositions of $\xi_{1}$ and $\xi_{2}$ respectively. Let also $\mathcal{A}_{1}$ be the set of 3-off-diagonal joinings in $J_{3}^{e}(\mathcal{T})$ and $\mathcal{A}_{2}$ be the set of 3 -off-diagonal joinings in $J_{3}^{e}(\mathcal{S})$. In view of Souslin theorem the sets $\mathcal{A}_{1}$ and $\mathcal{A}_{2}$ are measurable. We can assume that $\kappa_{1}\left(\mathcal{A}_{1}\right)=\kappa_{2}\left(\mathcal{A}_{2}\right)=0$. Indeed, let $\beta:=1-\kappa_{1}\left(\mathcal{A}_{1}\right) \geq 0$ and $\beta^{\prime}:=1-\kappa_{2}\left(\mathcal{A}_{2}\right) \geq 0$. Then

$$
\xi_{1}=(1-\beta) \int_{\mathbb{R}^{2}} \mu_{-t,-u} d P^{\prime}(t, u)+\beta \xi_{1}^{\prime}
$$

and

$$
\xi_{2}=\left(1-\beta^{\prime}\right) \int_{\mathbb{R}^{2}} \nu_{-t,-u} d Q^{\prime}(t, u)+\beta^{\prime} \xi_{2}^{\prime},
$$

where $\xi_{1}^{\prime} \in J_{3}(\mathcal{T})$ and $\xi_{2}^{\prime} \in J_{3}(\mathcal{S})$ do not have 3-off-diagonal joinings in their ergodic decomposition. Then

$$
\begin{aligned}
\mu_{a_{n}, b_{n}} & \rightarrow(1-\alpha) \int_{\mathbb{R}^{2}} \mu_{-t,-u} d P(t, u)+\alpha\left((1-\beta) \int_{\mathbb{R}^{2}} \mu_{-t,-u} d P^{\prime}(t, u)+\beta \xi_{1}^{\prime}\right) \\
& =(1-\alpha \beta) \int_{\mathbb{R}^{2}} \mu_{-t,-u} d\left(\frac{1-\alpha}{1-\alpha \beta} P+\frac{\alpha(1-\beta)}{1-\alpha \beta} P^{\prime}\right)+\alpha \beta \xi_{1}^{\prime} \\
& =(1-\alpha \beta) \int_{\mathbb{R}^{2}} \mu_{-t,-u} d \bar{P}+\alpha \beta \xi_{1}^{\prime}
\end{aligned}
$$

where $\bar{P}=\frac{1-\alpha}{1-\alpha \beta} P+\frac{\alpha(1-\beta)}{1-\alpha \beta} P^{\prime}$. Analogously

$$
\nu_{a_{n}, b_{n}} \rightarrow\left(1-\alpha^{\prime} \beta^{\prime}\right) \int_{\mathbb{R}^{2}} \nu_{-t,-u} d \bar{Q}+\alpha^{\prime} \beta^{\prime} \xi_{2}^{\prime},
$$

where $\bar{Q}=\frac{1-\alpha^{\prime}}{1-\alpha^{\prime} \beta^{\prime}} Q+\frac{\alpha^{\prime}\left(1-\beta^{\prime}\right)}{1-\alpha^{\prime} \beta^{\prime}} Q^{\prime}$. Then for the set $B$ satisfying 13 we have

$$
\begin{aligned}
& (1-\alpha \beta) \bar{P}(B)-\left(1-\alpha^{\prime} \beta^{\prime}\right) \bar{Q}(B) \\
& \quad=(1-\alpha) P(B)+\alpha(1-\beta) P^{\prime}(B)-\left(1-\alpha^{\prime}\right) Q(B)-\alpha^{\prime}\left(1-\beta^{\prime}\right) Q^{\prime}(B) \\
& \quad>\alpha^{\prime}+\alpha(1-\beta) P^{\prime}(B)-\alpha^{\prime}\left(1-\beta^{\prime}\right) Q^{\prime}(B) \geq \alpha^{\prime}-\alpha^{\prime}\left(1-\beta^{\prime}\right)=\alpha^{\prime} \beta^{\prime} .
\end{aligned}
$$

It is enough then, to replace $P, Q$ by $\bar{P}, \bar{Q}$ and $\alpha, \alpha^{\prime}$ by $\alpha \beta, \alpha^{\prime} \beta^{\prime}$ respectively.

Let $\lambda \in J^{e}(\mathcal{T}, \mathcal{S})$. We show that $\lambda=\mu \otimes \nu$. Consider the sequence $\left\{\lambda_{a_{n}, b_{n}}\right\}_{n \in \mathbb{N}}$ in $J_{3}^{e}(\mathcal{T} \times$ $\mathcal{S}, \lambda)$. By the compactness of $J_{3}(\mathcal{T} \times \mathcal{S}, \lambda)$ we have that $\lambda_{a_{n}, b_{n}} \rightarrow \eta$ weakly in $J_{3}(\mathcal{T} \times \mathcal{S}, \lambda)$, up to taking a subsequence. Moreover, by assumptions we have

and

$$
\left.\eta\right|_{X_{1} \times X_{2} \times X_{3}}=(1-\alpha) \int_{\mathbb{R}^{2}} \mu_{-t,-u} d P(t, u)+\alpha \xi_{1}
$$

$$
\left.\eta\right|_{Y_{1} \times Y_{2} \times Y_{3}}=\left(1-\alpha^{\prime}\right) \int_{\mathbb{R}^{2}} \nu_{-t,-u} d Q(t, u)+\alpha^{\prime} \xi_{2} .
$$

Let $h^{\mathcal{T}}: \mathbb{R}^{2} \rightarrow \mathcal{A}_{1}$ and $h^{\mathcal{S}}: \mathbb{R}^{2} \rightarrow \mathcal{A}_{2}$ be given by $h^{\mathcal{T}}(t, u):=\mu_{-t,-u}$ and $h^{\mathcal{S}}(t, u):=\nu_{-t,-u}$. Then

$$
\left.\eta\right|_{X_{1} \times X_{2} \times X_{3}}=\int_{J_{3}^{e}(\mathcal{T})} \rho^{\mathcal{T}} d\left((1-\alpha) h_{*}^{\mathcal{T} P}+\alpha \kappa_{1}\right)\left(\rho^{\mathcal{T}}\right),
$$

and

$$
\left.\eta\right|_{Y_{1} \times Y_{2} \times Y_{3}}=\int_{J_{3}^{e}(\mathcal{S})} \rho^{\mathcal{S}} d\left(\left(1-\alpha^{\prime}\right) h_{*}^{\mathcal{S}} Q+\alpha^{\prime} \kappa_{2}\right)\left(\rho^{\mathcal{S}}\right) .
$$

Let now $\eta=\int_{J_{3}^{e}(\mathcal{T} \times \mathcal{S}, \lambda)} \psi d \kappa(\psi)$ be the ergodic decomposition of $\eta$. Then we have

$$
\left.\eta\right|_{X_{1} \times X_{2} \times X_{3}}=\left.\int_{J_{3}^{e}(\mathcal{T} \times \mathcal{S}, \lambda)} \psi\right|_{X_{1} \times X_{2} \times X_{3}} d \kappa(\psi),
$$


and

$$
\left.\eta\right|_{Y_{1} \times Y_{2} \times Y_{3}}=\left.\int_{J_{3}^{e}(\mathcal{T} \times \mathcal{S}, \lambda)} \psi\right|_{Y_{1} \times Y_{2} \times Y_{3}} d \kappa(\psi) .
$$

Since $\psi \in J_{3}^{e}(\mathcal{T} \times \mathcal{S})$, we have $\left.\psi\right|_{X_{1} \times X_{2} \times X_{3}} \in J_{3}^{e}(\mathcal{T})$ and $\left.\psi\right|_{Y_{1} \times Y_{2} \times Y_{3}} \in J_{3}^{e}(\mathcal{S})$. Consider $\Omega^{\mathcal{T}}: J_{3}^{e}(\mathcal{T} \times \mathcal{S}, \lambda) \rightarrow J_{3}^{e}(\mathcal{T})$ and $\Omega^{\mathcal{S}}: J_{3}^{e}(\mathcal{T} \times \mathcal{S}, \lambda) \rightarrow J_{3}^{e}(\mathcal{S})$ given by

$$
\Omega^{\mathcal{T}}(\psi)=\left.\psi\right|_{X_{1} \times X_{2} \times X_{3}} \quad \text { and } \quad \Omega^{\mathcal{S}}(\psi)=\left.\psi\right|_{Y_{1} \times Y_{2} \times Y_{3}} .
$$

We have

$$
\left.\eta\right|_{X_{1} \times X_{2} \times X_{3}}=\int_{J_{3}^{e}(\mathcal{T})} \rho^{\mathcal{T}} d\left(\Omega_{*}^{\mathcal{T}} \kappa\right)\left(\rho^{\mathcal{T}}\right),
$$

and

$$
\left.\eta\right|_{Y_{1} \times Y_{2} \times Y_{3}}=\int_{J_{3}^{e}(\mathcal{S})} \rho^{\mathcal{S}} d\left(\Omega_{*}^{\mathcal{S}} \kappa\right)\left(\rho^{\mathcal{S}}\right) .
$$

By comparing this with (14) and 15 and using the uniqueness of ergodic decomposition we obtain that

$$
\Omega_{*}^{\mathcal{T}} \kappa=(1-\alpha) h_{*}^{\mathcal{T}} P+\alpha \kappa_{1} \quad \text { and } \quad \Omega_{*}^{\mathcal{S}} \kappa=\left(1-\alpha^{\prime}\right) h_{*}^{\mathcal{S}} Q+\alpha^{\prime} \kappa_{2}
$$

Let now

$$
\begin{aligned}
\mathcal{A}:=\left\{\psi \in J_{3}^{e}(\mathcal{T} \times \mathcal{S}, \lambda): \exists t, u, t^{\prime}, u^{\prime} \in \mathbb{R},(t, u)\right. & \neq\left(t^{\prime}, u^{\prime}\right), \\
& \left.\left.\psi\right|_{X_{1} \times X_{2} \times X_{3}}=\mu_{-t,-u},\left.\psi\right|_{Y_{1} \times Y_{2} \times Y_{3}}=\nu_{-t^{\prime},-u^{\prime}}\right\} .
\end{aligned}
$$

We now show that $\kappa(\mathcal{A})>0$. For any measurable subsets $C \subset J_{3}^{e}(\mathcal{T})$ and $D \subset J_{3}^{e}(\mathcal{S})$ denote by $C \overline{\times} D$ the set of all $\psi \in J_{3}^{e}(\mathcal{T} \times \mathcal{S}, \lambda)$ such that $\left.\psi\right|_{X_{1} \times X_{2} \times X_{3}} \in C$ and $\psi_{Y_{1} \times Y_{2} \times Y_{3}} \in D$.

Assume that $\kappa(\mathcal{A})=0$. Let $B$ be the set satisfying $(13)$. If $(t, u) \in B$ then by the definition of $h^{\mathcal{T}}$ and $h^{\mathcal{S}}$ we have $\mu_{-t,-u} \in h^{\mathcal{T}}(B)$ and $\nu_{-t,-u} \in h^{\mathcal{S}}(\bar{B})$. Moreover $\kappa(\mathcal{A})=0$ and $h^{\mathcal{T}}(B) \overline{\times}\left(\mathcal{A}_{2} \backslash\right.$ $\left.h^{\mathcal{S}}(B)\right) \subset \mathcal{A}$ yield

$$
\kappa\left(h^{\mathcal{T}}(B) \overline{\times} \mathcal{A}_{2}\right)=\kappa\left(h^{\mathcal{T}}(B) \overline{\times} h^{\mathcal{S}}(B)\right) .
$$

Note that $\kappa_{1}\left(h^{\mathcal{T}}(B)\right) \leq \kappa_{1}\left(\mathcal{A}_{1}\right)=0$. Hence, 16 and 17 implies

$$
\begin{aligned}
(1-\alpha) P(B) & =(1-\alpha) h_{*}^{\mathcal{T}} P\left(h^{\mathcal{T}}(B)\right)=\left[(1-\alpha) h_{*}^{\mathcal{T}} P+\alpha \kappa_{1}\right]\left(h^{\mathcal{T}}(B)\right) \\
& =\Omega_{*}^{\mathcal{T}} \kappa\left(h^{\mathcal{T}}(B)\right)=\kappa\left(h^{\mathcal{T}}(B) \overline{\times} J_{3}^{e}(\mathcal{S})\right) \\
& =\kappa\left(h^{\mathcal{T}}(B) \overline{\times} \mathcal{A}_{2}\right)+\kappa\left(h^{\mathcal{T}}(B) \overline{\times}\left(J_{3}^{e}(\mathcal{S}) \backslash \mathcal{A}_{2}\right)\right) \\
& =\kappa\left(h^{\mathcal{T}}(B) \overline{\times} h^{\mathcal{S}}(B)\right)+\kappa\left(h^{\mathcal{T}}(B) \overline{\times}\left(J_{3}^{e}(\mathcal{S}) \backslash \mathcal{A}_{2}\right)\right) .
\end{aligned}
$$

Analogously we also obtain

$$
\left(1-\alpha^{\prime}\right) Q(B)=\left(1-\alpha^{\prime}\right) h_{*}^{\mathcal{S}} Q\left(h^{\mathcal{S}}(B)\right)=\kappa\left(h^{\mathcal{T}}(B) \overline{\times} h^{\mathcal{S}}(B)\right)+\kappa\left(\left(J_{3}^{e}(\mathcal{T}) \backslash \mathcal{A}_{1}\right) \overline{\times} h^{\mathcal{S}}(B)\right)
$$

Moreover, in view of 16 we get

$$
\begin{aligned}
\kappa\left(h^{\mathcal{T}}(B) \overline{\times}\left(J_{3}^{e}(\mathcal{S}) \backslash \mathcal{A}_{2}\right)\right) & \leq \kappa\left(J_{3}^{e}(\mathcal{T}) \overline{\times}\left(J_{3}^{e}(\mathcal{S}) \backslash \mathcal{A}_{2}\right)\right) \\
& =\Omega_{*}^{\mathcal{S}} \kappa\left(J_{3}^{e}(\mathcal{S}) \backslash \mathcal{A}_{2}\right)=\alpha^{\prime} \kappa_{2}\left(J_{3}^{e}(\mathcal{S}) \backslash \mathcal{A}_{2}\right)=\alpha^{\prime} .
\end{aligned}
$$

Since $(1-\alpha) P(B)-\left(1-\alpha^{\prime}\right) Q(B)>\alpha^{\prime}$, by substracting 18 and 19 we obtain

$$
\begin{aligned}
\alpha^{\prime} & <(1-\alpha) h_{*}^{\mathcal{T}} P\left(h^{\mathcal{T}}(B)\right)-\left(1-\alpha^{\prime}\right) h_{*}^{\mathcal{T}} Q\left(h^{\mathcal{S}}(B)\right) \\
& =\left(\kappa\left(h^{\mathcal{T}}(B) \overline{\times} h^{\mathcal{S}}(B)\right)+\kappa\left(h^{\mathcal{T}}(B) \overline{\times}\left(J_{3}^{e}(\mathcal{S}) \backslash \mathcal{A}_{2}\right)\right)\right) \\
& -\left(\kappa\left(h^{\mathcal{T}}(B) \overline{\times} h^{\mathcal{S}}(B)\right)+\kappa\left(\left(J_{3}^{e}(\mathcal{T}) \backslash \mathcal{A}_{1}\right) \overline{\times} h^{\mathcal{S}}(B)\right)\right) \\
& =\kappa\left(h^{\mathcal{T}}(B) \overline{\times}\left(J_{3}^{e}(\mathcal{S}) \backslash \mathcal{A}_{2}\right)\right)-\kappa\left(\left(J_{3}^{e}(\mathcal{T}) \backslash \mathcal{A}_{1}\right) \overline{\times} h^{\mathcal{S}}(B)\right) \leq \alpha^{\prime},
\end{aligned}
$$

which is a contradiction. This yields $\kappa(\mathcal{A})>0$ and hence $\mathcal{A}$ is non-empty. Therefore, there exists $\psi \in \mathcal{A} \subset J_{3}^{e}(\mathcal{T} \times \mathcal{S}, \lambda)$ such that $\left.\psi\right|_{X_{1} \times X_{2} \times X_{3}}=\mu_{t, u}$ and $\left.\psi\right|_{Y_{1} \times Y_{2} \times Y_{3}}=\nu_{t^{\prime}, u^{\prime}}$ with $(t, u) \neq\left(t^{\prime}, u^{\prime}\right)$. 
Assume that $t \neq t^{\prime}$ (the case when $u \neq u^{\prime}$ is analogous). Then $\phi:=\Pi_{1,3}(\psi) \in J_{2}^{e}(\mathcal{T} \times \mathcal{S}, \lambda)$ satisfies

$$
\left.\phi\right|_{X_{1} \times X_{3}}=\mu_{t} \quad \text { and }\left.\quad \phi\right|_{Y_{1} \times Y_{3}}=\nu_{t^{\prime}}
$$

Thus, by Lemma 3.1, $\lambda=\mu \otimes \nu$.

The above criterion strengthens the results obtained in [4], that is the flows described in this paper are not only non-isomorphic with their inverses, but also disjoint. To prove the main result of this paper, we use the following simplified version of Proposition 3.2

Corollary 3.4. Let $\mathcal{T}=\left\{T_{t}\right\}_{t \in \mathbb{R}}$ and $\mathcal{S}=\left\{S_{t}\right\}_{t \in \mathbb{R}}$ be weakly mixing flows acting on the standard Borel spaces $(X, \mathcal{B}, \mu)$ and $(Y, \mathcal{C}, \nu)$ respectively. Assume that for some real sequences $\left(a_{n}\right)_{n \in \mathbb{N}}$ and $\left(b_{n}\right)_{n \in \mathbb{N}}$ we have

$$
\mu_{a_{n}, b_{n}} \rightarrow \int_{\mathbb{R}^{2}} \mu_{-t,-u} d P(t, u) \text { and } \nu_{a_{n}, b_{n}} \rightarrow \int_{\mathbb{R}^{2}} \nu_{-t,-u} d Q(t, u),
$$

for some measures $P, Q \in \mathcal{P}\left(\mathbb{R}^{2}\right)$. If $P \neq Q$, then $\mathcal{T}$ and $\mathcal{S}$ are disjoint.

Let $\xi: \mathbb{R}^{2} \rightarrow \mathbb{R}$ be given by $\xi(t, u):=t-2 u$. The following result gives a condition on limit joinings which imply weak mixing of a flow.

Proposition 3.5. Let $\mathcal{T}=\left\{T_{t}\right\}_{t \in \mathbb{R}}$ be an ergodic flow on $(X, \mathcal{B}, \mu)$ and assume that there exists a real increasing sequence $\left\{b_{n}\right\}_{n \in \mathbb{N}}$, a real number $\rho \in[0,1)$ and a probability measure $P \in \mathcal{P}\left(\mathbb{R}^{2}\right)$ such that

$$
\mu_{2 b_{n}, b_{n}} \rightarrow(1-\rho) \int_{\mathbb{R}^{2}} \mu_{-t,-u} d P(t, u)+\rho \psi,
$$

for some $\psi \in J_{3}(\mathcal{T})$. If $P$ is not supported on an affine lattice in $\mathbb{R}^{2}$ then $\mathcal{T}$ is weakly mixing. In particular, if there exist two rationally independent real numbers $d_{1}$ and $d_{2}$ such that $d_{1}, d_{2}$ and 0 are atoms of $\xi_{*} P$, then the flow $\mathcal{T}$ is weakly mixing.

Proof. Assume that $P$ is not supported on an affine lattice and the flow $\mathcal{T}$ is not weakly mixing. Then there exists a non-zero function $f \in L^{2}(X, \mu)$ and $a \in \mathbb{R} \backslash\{0\}$ such that

$$
\forall t \in \mathbb{R}, f \circ T_{t}=e^{-2 \pi i a t} f .
$$

Recall that $\sigma_{1}: \mathbb{R}^{2} \rightarrow \mathbb{R}$ denotes the projection on the first coordinate. By applying $\Psi \circ \Pi_{1,3}$ (see (3) to (20) and using (4) and (5), we obtain

$$
T_{2 b_{n}} \rightarrow(1-\rho) \int_{\mathbb{R}} T_{t} d P_{1}(t)+\rho \Psi_{1},
$$

where $P_{1}:=\left(\sigma_{1}\right)_{*} P$ and $\Psi_{1}$ is a Markov operator. Let $\langle\cdot, \cdot\rangle$ be the scalar product on $L^{2}(X, \mu)$. By 21), we get

$$
\|f\|^{2}=|\langle f, f\rangle|=\left|\left\langle f, e^{-2 \pi i a t} f\right\rangle\right|=\left|\left\langle f, f \circ T_{t}\right\rangle\right|=\left|\left\langle f, f \circ T_{2 b_{n}}\right\rangle\right|
$$

for every $n \in \mathbb{N}$. As $n \rightarrow \infty$, we get

$$
\|f\|^{2}=\left|\left\langle f, f \circ T_{2 b_{n}}\right\rangle\right|=\left|\left\langle f,(1-\rho) \int_{\mathbb{R}} f \circ T_{t} d P_{1}(t)+\rho \Psi_{1}(f)\right\rangle\right| .
$$


On the other hand by the fact that Markov operator is a contraction, we get

$$
\begin{aligned}
& \left|\left\langle f,(1-\rho) \int_{\mathbb{R}} f \circ T_{t} d P_{1}(t)+\rho \Psi_{1}(f)\right\rangle\right| \\
& \quad \leq(1-\rho)\left|\left\langle f, \int_{\mathbb{R}} f \circ T_{t} d P_{1}(t)\right\rangle\right|+\rho\left|\left\langle f, \Psi_{1}(f)\right\rangle\right| \\
& \quad \leq(1-\rho)\left|\int_{\mathbb{R}}\left\langle f, f \circ T_{t}\right\rangle d P_{1}(t)\right|+\rho\|f\|^{2} \\
& \quad=(1-\rho)\|f\|^{2}\left|\int_{\mathbb{R}} e^{-2 \pi i a t} d P_{1}(t)\right|+\rho\|f\|^{2}
\end{aligned}
$$

Thus we get

$$
\left|\int_{\mathbb{R}} e^{-2 \pi i a t} d P_{1}(t)\right|=1
$$

that is

$$
\int_{\mathbb{R}} e^{-2 \pi i a t} d P_{1}(t)=e^{-2 \pi i b} \text { for some } b \in \mathbb{R} \text {. }
$$

It follows that

$$
\int_{\mathbb{R}} e^{-2 \pi i(a t-b)} d P_{1}(t)=1
$$

This implies

$$
P_{1}(\{t \in \mathbb{R} ; a t-b \in \mathbb{Z}\})=1 .
$$

Consider now $P_{2}:=\left(\sigma_{2}\right)_{*} P$. Analogously, by applying $\Psi \circ \Pi_{2,3}$ to 20 , we get

$$
P_{2}(\{u \in \mathbb{R} ; a u-c \in \mathbb{Z}\})=1 \text { for some } c \in \mathbb{R} .
$$

Combining the two above results, we finally obtain

$$
P\left(\left\{(t, u) \in \mathbb{R}^{2} ; a(t, u)-(b, c) \in \mathbb{Z}^{2}\right\}\right)=1,
$$

which is a contradiction with our assumption. Thus if $P$ is not supported on an affine lattice then the flow $\mathcal{T}$ is weakly mixing.

Suppose now that $\xi_{*} P$ has atoms at points $0, d_{1}$ and $d_{2}$. Assume again that $\mathcal{T}$ is not weakly mixing and that $e^{2 \pi i a}, a \neq 0$, is an eigenvalue. By the definition of $\xi$, the lines $\left(x, \frac{1}{2}\left(x-d_{i}\right)\right)$ for $i=1,2$ and $\left(x, \frac{1}{2} x\right)$ have positive measure $P$. This together with 22 yields $x_{0}, x_{1}, x_{2} \in \mathbb{R}$, such that

$$
\begin{aligned}
& a\left(x_{0}, \frac{1}{2} x_{0}\right)-(b, c) \in \mathbb{Z}^{2}, \\
& a\left(x_{1}, \frac{1}{2}\left(x_{1}-d_{1}\right)\right)-(b, c) \in \mathbb{Z}^{2}, \\
& a\left(x_{2}, \frac{1}{2}\left(x_{2}-d_{2}\right)\right)-(b, c) \in \mathbb{Z}^{2} .
\end{aligned}
$$

This implies

$$
\begin{aligned}
& a\left(x_{1}-x_{0}, \frac{1}{2}\left(x_{1}-x_{0}\right)-\frac{1}{2} d_{1}\right) \in \mathbb{Z}^{2}, \\
& a\left(x_{2}-x_{0}, \frac{1}{2}\left(x_{2}-x_{0}\right)-\frac{1}{2} d_{2}\right) \in \mathbb{Z}^{2} .
\end{aligned}
$$

By applying $\xi$ to the above, we get that $a d_{1} \in \mathbb{Z}$ and $a d_{2} \in \mathbb{Z}$. Since $a, d_{1}, d_{2} \neq 0$, we get that here $\left(a d_{1}\right) d_{2}-\left(a d_{2}\right) d_{1}=0$ is a non-trivial integer combination of $d_{1}$ and $d_{2}$. By the rational independence of $d_{1}$ and $d_{2}$ this yields $a=0$. This is a contradiction, hence $\mathcal{T}$ is weakly mixing. 


\section{Acceptable permutations}

In this section, we establish a technical result concerning a particular non-degenerate permutation, which plays a key role in proving that our main result applies to all non-hyperelliptic connected components of the moduli space. In particular, in view of the Remark 2.10, we assume that the alphabet we consider has $d \geq 6$ elements. Recall that in every Rauzy class we can fix a non-degenerate permutation $\pi=\left\{\pi_{0}, \pi_{1}\right\}$ satisfying

$$
\pi_{1} \circ \pi_{0}^{-1}(1)=d \quad \text { and } \quad \pi_{1} \circ \pi_{0}^{-1}(d)=1 .
$$

We have the following theorem.

Proposition 4.1. In every Rauzy class corresponding to a non-hyperelliptic connected component of the moduli space $\mathcal{M}$, there exists a permutation $\pi=\left\{\pi_{0}, \pi_{1}\right\}$ satisfying (23) such that there exist distinct symbols $\alpha_{1}, \alpha_{2}, \gamma_{1}, \gamma_{2} \in \mathcal{A} \backslash\left\{\pi_{0}^{-1}(1), \pi_{0}^{-1}(d)\right\}$ satisfying the three following properties

$$
\begin{aligned}
& \Omega_{\alpha_{1} \alpha_{2}}=\Omega_{\alpha_{2} \alpha_{1}}=0, \\
& \Omega_{\alpha_{1} \gamma_{2}} \Omega_{\alpha_{2} \gamma_{1}}=0 \\
& \Omega_{\alpha_{1} \gamma_{1}} \Omega_{\alpha_{2} \gamma_{2}} \neq 0,
\end{aligned}
$$

where $\Omega:=\Omega_{\pi}$ is the associated translation matrix.

Proof. Let $\pi=\left\{\pi_{0}, \pi_{1}\right\}$ be a non-degenerate permutation satisfying (23) that belongs to a Rauzy class associated with a non-hyperelliptic connected component. Then it is not symmetric, hence its translation matrix $\Omega$ contains zero entries outside the diagonal. Indeed, assume contrary to our claim that

$$
\pi_{0}(\alpha)<\pi_{0}(\beta) \Leftrightarrow \pi_{1}(\alpha)>\pi_{1}(\beta) \text { for all } \alpha, \beta \in \mathcal{A} .
$$

Then for every $\alpha \in \mathcal{A}$

$$
\pi_{1}(\alpha)=\#\left\{\beta \in \mathcal{A} ; \pi_{1}(\beta)<\pi_{1}(\alpha)\right\}+1=\#\left\{\beta \in \mathcal{A} ; \pi_{0}(\beta)>\pi_{0}(\alpha)\right\}+1=d-\pi_{0}(\alpha)+1 .
$$

Hence $\pi$ is a symmetric permutation.

We need to consider two cases separately.

Case 1. Assume first that there exists a symbol $\alpha \in \mathcal{A}$ such that for all symbols $\beta \in \mathcal{A}$ with $1<\pi_{0}(\beta)<d$ we have

that is

$$
\pi_{0}(\delta)<\pi_{0}(\alpha) \Leftrightarrow \pi_{1}(\delta)<\pi_{1}(\alpha)
$$

$$
\Omega_{\alpha \beta}=0 \quad \text { for all } \beta \in \mathcal{A} \backslash\left\{\pi_{0}^{-1}(1), \pi_{0}^{-1}(d)\right\} .
$$

Since $\pi$ is non-degenerate, there exist symbols $\alpha_{1}, \gamma_{1}$ such that

$$
1<\pi_{0}\left(\alpha_{1}\right)<\pi_{0}\left(\gamma_{1}\right)<\pi_{0}(\alpha) \text { and } 1<\pi_{1}\left(\gamma_{1}\right)<\pi_{1}\left(\alpha_{1}\right)<\pi_{1}(\alpha) .
$$

Otherwise, $\pi$ satisfies $\left(9\right.$ and hence, it is degenerate. Similarly, there exist symbols $\alpha_{2}, \gamma_{2}$ such that

$$
d>\pi_{0}\left(\alpha_{2}\right)>\pi_{0}\left(\gamma_{2}\right)>\pi_{0}(\alpha) \text { and } d>\pi_{1}\left(\gamma_{2}\right)>\pi_{1}\left(\alpha_{2}\right)>\pi_{1}(\alpha) .
$$

Otherwise, $\pi$ satisfies 10 and it is again degenerate. Thus we have

$$
\Omega_{\alpha_{1} \alpha_{2}}=\Omega_{\alpha_{2} \alpha_{1}}=\Omega_{\alpha_{1} \gamma_{2}}=\Omega_{\alpha_{2} \gamma_{1}}=0 \text { and } \Omega_{\alpha_{1} \gamma_{1}}=1 \text { and } \Omega_{\alpha_{2} \gamma_{2}}=-1,
$$

which is the desired property. Hence $\alpha_{1}, \alpha_{2}, \gamma_{1}, \gamma_{2}$ are the desired symbols.

Case 2. Assume now, that there are no symbols satisfying (25). Since there are zeroes outside the diagonal in $\Omega_{\pi}$, there exist two distinct symbols $\alpha_{1}, \alpha_{2} \in \mathcal{A}$ such that $\Omega_{\alpha_{1} \alpha_{2}}=\Omega_{\alpha_{2} \alpha_{1}}=0$. Case 2a. Suppose first that the rows of $\Omega_{\pi}$ corresponding to $\alpha_{1}$ and $\alpha_{2}$ are not identical. Then there exists a symbol $\gamma$ such that $\Omega_{\alpha_{1} \gamma} \neq 0$ and $\Omega_{\alpha_{2} \gamma}=0$ or $\Omega_{\alpha_{2} \gamma} \neq 0$ and $\Omega_{\alpha_{1} \gamma}=0$. Assume that the first case holds (the second is done analogously) and set $\gamma_{1}:=\gamma$. Note that $\gamma_{1} \in$ $\mathcal{A} \backslash\left\{\pi_{0}^{-1}(1), \pi_{0}^{-1}(d)\right\}$. Since $\alpha_{2}$ does not satisfy $(25)$, there exist two $\gamma_{2} \in \mathcal{A} \backslash\left\{\pi_{0}^{-1}(1), \pi_{0}^{-1}(d)\right\}$, $\gamma_{2} \neq \gamma_{1}$, such that $\Omega_{\alpha_{2} \gamma_{2}} \neq 0$. Thus we obtain (24). 
Case 2b. Suppose that the rows of the matrix $\Omega$ corresponding to indices $\alpha_{1}, \alpha_{2}$ are identical. Then there are no indices $\gamma_{1}, \gamma_{2}$ such that $\alpha_{1}, \alpha_{2}, \gamma_{1}, \gamma_{2}$ satisfy (24). We show that there is a different set of symbols satisfying (24). Note that all symbols $\beta$ such that

$$
\pi_{0}\left(\alpha_{1}\right)<\pi_{0}(\beta)<\pi_{0}\left(\alpha_{2}\right)
$$

satisfy

$$
\pi_{1}\left(\alpha_{1}\right)<\pi_{1}(\beta)<\pi_{1}\left(\alpha_{2}\right) .
$$

Otherwise only one of the entries $\Omega_{\alpha_{1} \beta}$ and $\Omega_{\alpha_{2} \beta}$ would be non-zero. In other words all symbols $\beta \in \mathcal{A}$ satisfying (26) satisfy $\Omega_{\alpha_{1} \beta}=\Omega_{\alpha_{2} \beta}=0$. Observe that there exist two different symbols $\hat{\alpha}_{1}, \gamma_{1} \in \mathcal{A}$ satisfying (26) such that $\Omega_{\hat{\alpha}_{1} \gamma_{1}} \neq 0$. Otherwise the permutation $\pi$ satisfies (8) and it is degenerate. Since $\alpha_{1}, \alpha_{2}$ do not satisfy (25) and the corresponding rows are identical, there exists a symbol $\gamma_{2} \in \mathcal{A}$ such that $\Omega_{\alpha_{1} \gamma_{2}}=\Omega_{\alpha_{2} \gamma_{2}} \neq 0$. It follows that $\Omega_{\hat{\alpha}_{1} \gamma_{2}}=\Omega_{\alpha_{2} \gamma_{2}} \neq 0$. We have

$$
\Omega_{\hat{\alpha}_{1} \alpha_{2}}=\Omega_{\alpha_{2} \hat{\alpha}_{1}}=\Omega_{\alpha_{2} \gamma_{1}}=0 \text { and } \Omega_{\hat{\alpha}_{1} \gamma_{1}} \neq 0 \text { and } \Omega_{\alpha_{2} \gamma_{2}} \neq 0 \text {. }
$$

Hence, $\hat{\alpha}_{1}, \alpha_{2}, \gamma_{1}, \gamma_{2}$ are the desired symbols.

Corollary 4.2. If $\pi$ is a nonsymmetric and nondegenerate permutation satisfying (23), and $\tau \in \mathbb{R}^{\mathcal{A}}$ is a rationally independent vector, then there exist $\alpha_{1}, \alpha_{2} \in \mathcal{A}$ such that $\Omega_{\alpha_{1} \alpha_{2}}=$ $\Omega_{\alpha_{2} \alpha_{1}}=0$ and for each $i=1,2$ the numbers

$$
(\Omega \tau)_{\alpha_{2}}-(\Omega \tau)_{\alpha_{1}}, \text { and }(\Omega \tau)_{\alpha_{i}}-\left((\Omega \tau)_{\pi_{0}^{-1}(1)}+(\Omega \tau)_{\pi_{0}^{-1}(d)}\right)
$$

are rationally independent.

Proof. We prove the case when $i=1$. If $i=2$, the proof goes along the same lines. Consider symbols $\alpha_{1}, \alpha_{2}, \gamma_{1}, \gamma_{2}$ given by Proposition 4.1 . We have $\Omega_{\alpha_{1} \alpha_{2}}=\Omega_{\alpha_{2} \alpha_{1}}=0$. Assume that there exist integers $p$ and $q$ such that

$$
\begin{aligned}
0 & =p\left((\Omega \tau)_{\alpha_{2}}-(\Omega \tau)_{\alpha_{1}}\right)+q\left((\Omega \tau)_{\alpha_{1}}-\left((\Omega \tau)_{\pi_{0}^{-1}(1)}+(\Omega \tau)_{\pi_{0}^{-1}(d)}\right)\right) \\
& =\sum_{\beta \in \mathcal{A}}\left(-q \Omega_{\pi_{0}^{-1}(1) \beta}-q \Omega_{\pi_{0}^{-1}(d) \beta}+(q-p) \Omega_{\alpha_{1} \beta}+p \Omega_{\alpha_{2} \beta}\right) \tau_{\beta}
\end{aligned}
$$

By rational independence of $\tau$, this yields

$$
-q \Omega_{\pi_{0}^{-1}(1) \beta}-q \Omega_{\pi_{0}^{-1}(d) \beta}+(q-p) \Omega_{\alpha_{1} \beta}+q \Omega_{\alpha_{2} \beta}=0,
$$

for every $\beta \in \mathcal{A}$. Since

$$
\Omega_{\pi_{0}^{-1}(1) \beta}=1 \text { for } \beta \in \mathcal{A} \backslash\left\{\pi_{0}^{-1}(1)\right\} \text { and } \Omega_{\pi_{0}^{-1}(d) \beta}=-1 \text { for } \beta \in \mathcal{A} \backslash\left\{\pi_{0}^{-1}(d)\right\},
$$

we have

$$
(q-p) \Omega_{\alpha_{1} \beta}+q \Omega_{\alpha_{2} \beta}=0 \text { for } \beta \in \mathcal{A} \backslash\left\{\pi_{0}^{-1}(1), \pi_{0}^{-1}(d)\right\}
$$

Since by 24 the matrix

$$
\left[\begin{array}{ll}
\Omega_{\alpha_{1} \gamma_{1}} & \Omega_{\alpha_{1} \gamma_{2}} \\
\Omega_{\alpha_{2} \gamma_{1}} & \Omega_{\alpha_{2} \gamma_{2}}
\end{array}\right]
$$

is triangular and has non-zero entries on the main diagonal, it follows that

$$
p=q=0,
$$

which proves the rational independence. 


\section{The Measures on THE SURFACE}

In this section we will deal with measures on a given surface $(M, \Sigma)$ which are absolutely continuous with respect to the Lebesgue measure. We want to prove that, if the density of such measure is bounded and close enough to the constant function 1 in $L^{1}$, then there is an explicit way to construct a homeomorphism which pushes this measure to the Lebesgue measure. The computation given below is partially inspired by the paper of Moser [19]. We will need the following auxiliary lemma.

Lemma 5.1. Let $x, y \in \mathbb{R}^{2}$ be two points on the plane and let $\overline{x y}$ be the segment with endpoints at $x$ and $y$. Let $H_{1}, H_{2}$ be two affine transformations on $\mathbb{R}^{2}$. If $H_{1}(x)=H_{2}(x)$ and $H_{1}(y)=H_{2}(y)$, then $\left.H_{1}\right|_{\overline{x y}}=\left.H_{2}\right|_{\overline{x y}}$. Moreover, for each noncollinear triples $x_{1}, x_{2}, x_{3} \in \mathbb{R}^{2}$ and $y_{1}, y_{2}, y_{3} \in \mathbb{R}^{2}$ there exists a unique invertible affine transformation $H$ such that $H\left(x_{i}\right)=y_{i}$ for $i=1,2,3$.

We will now prove lemmas which give a construction of a homeomorphism of an isosceles right triangle which pushes forward any given absolutely continuous measure whose density satisfies some conditions to the Lebesgue measure. For any affine transformation $G: \mathbb{R}^{2} \rightarrow \mathbb{R}^{2}$ define $\operatorname{lin}(G)=D G$ as the matrix determining its linear part, $D$ denotes the derivative. Moreover for any real $2 \times 2$ matrix $M=\left(\begin{array}{ll}a & b \\ c & d\end{array}\right)$ we have the following formula for the operator norm

$$
\|M\|:=\sqrt{\frac{a^{2}+b^{2}+c^{2}+d^{2}+\sqrt{\left(a^{2}+b^{2}+c^{2}+d^{2}\right)^{2}-4(\operatorname{det}(M))^{2}}}{2}} .
$$

In particular, $\|\operatorname{lin}(G)\|$ is a Lipschitz constant of $G$.

On the space of homeomorphisms $\operatorname{Hom}(X)$ of a compact metric space $X$, we will consider the standard metric $d_{\text {Hom }}(H, G):=\max \left\{\sup _{x \in X} d(H(x), G(x)), \sup _{x \in X} d\left(H^{-1}(x), G^{-1}(x)\right)\right\}$, where $d$ is the metric on $X$.

Throughout this section we will heavily depend on the following construction. Let $0<a<1$, and let $V$ be the triangle in $\mathbb{R}^{2}$ with vertices in points $(0, a),(a, 0),(0,-a)$. Let $V_{1}$ and $V_{2}$ be the triangles whose vertices are $(0, a),(a, 0),(0,0)$ and $(0,0),(a, 0),(0,-a)$ respectively. Let $0 \leq h<1$ and $0<\epsilon<1$. Let $y(h):=(\epsilon a(1-h), h a)$. Consider the triangles given by the following sets of vertices:

- $C_{1}=C_{1}(h, \epsilon)$ given by $\{(0,0),(0, a), y(h, \epsilon)\}$;

- $C_{2}=C_{2}(h, \epsilon)$ given by $\{(a, 0),(0, a), y(h, \epsilon)\}$;

- $C_{3}=C_{3}(h, \epsilon)$ given by $\{(0,0),(\epsilon a, 0), y(h, \epsilon)\}$;

- $C_{4}=C_{4}(h, \epsilon)$ given by $\{(a, 0),(\epsilon a, 0), y(h, \epsilon)\}$;

- $C_{5}=C_{5}(h, \epsilon)$ given by $\{(0,0),(0,-a),(\epsilon a, 0)\}$;

- $C_{6}=C_{6}(h, \epsilon)$ given by $\{(a, 0),(0,-a),(\epsilon a, 0)\}$.

Let $\hat{h}:=\frac{h}{h+1} \geq 0$. Consider the point $\hat{y}(h, \epsilon)=(\epsilon a-\hat{h} \epsilon a,-\hat{h} a)$. Consider also the triangles

- $\hat{C}_{1}=\hat{C}_{1}(h, \epsilon)$ given by $\{(0,0),(0, a),(\epsilon a, 0)\}$;

- $\hat{C}_{2}=\hat{C}_{2}(h, \epsilon)$ given by $\{(a, 0),(0, a),(\epsilon a, 0)\}$;

- $\hat{C}_{3}=\hat{C}_{3}(h, \epsilon)$ given by $\{(0,0),(\epsilon a, 0), \hat{y}(h, \epsilon)\}$;

- $\hat{C}_{4}=\hat{C}_{4}(h, \epsilon)$ given by $\{(a, 0),(\epsilon a, 0), \hat{y}(h, \epsilon)\}$;

- $\hat{C}_{5}=\hat{C}_{5}(h, \epsilon)$ given by $\{(0,0),(0,-a), \hat{y}(h, \epsilon)\}$;

- $\hat{C}_{6}=\hat{C}_{6}(h, \epsilon)$ given by $\{(a, 0),(0,-a), \hat{y}(h, \epsilon)\}$.

By the definition of $h$ and $\hat{h}$ we have

$$
\begin{gathered}
\frac{\operatorname{Leb}\left(C_{1}\right)}{\operatorname{Leb}\left(\hat{C}_{1}\right)}=\frac{\operatorname{Leb}\left(C_{2}\right)}{\operatorname{Leb}\left(\hat{C}_{2}\right)}=1-h \quad \text { and } \\
\frac{\operatorname{Leb}\left(C_{3}\right)}{\operatorname{Leb}\left(\hat{C}_{3}\right)}=\frac{\operatorname{Leb}\left(C_{4}\right)}{\operatorname{Leb}\left(\hat{C}_{4}\right)}=\frac{\operatorname{Leb}\left(C_{5}\right)}{\operatorname{Leb}\left(\hat{C}_{5}\right)}=\frac{\operatorname{Leb}\left(C_{6}\right)}{\operatorname{Leb}\left(\hat{C}_{6}\right)}=1+h .
\end{gathered}
$$


Define $H(h, \epsilon): V \rightarrow V$ as a piecewise affine homeomorphism such that

$$
\begin{aligned}
\text { (i) } & H(h, \epsilon)\left(C_{i}\right)=\hat{C}_{i},\left.H(h, \epsilon)\right|_{C_{i}} \text { is affine for } i=1, \ldots, 6 \text {; } \\
\text { (ii) } & H(h, \epsilon) \text { fixes }(0,0),(0, a),(0,-a),(a, 0), \\
\text { (iii) } & H(h, \epsilon)(y)=(a \epsilon, 0) \text { and } H(h, \epsilon)(a \epsilon, 0)=\hat{y} .
\end{aligned}
$$
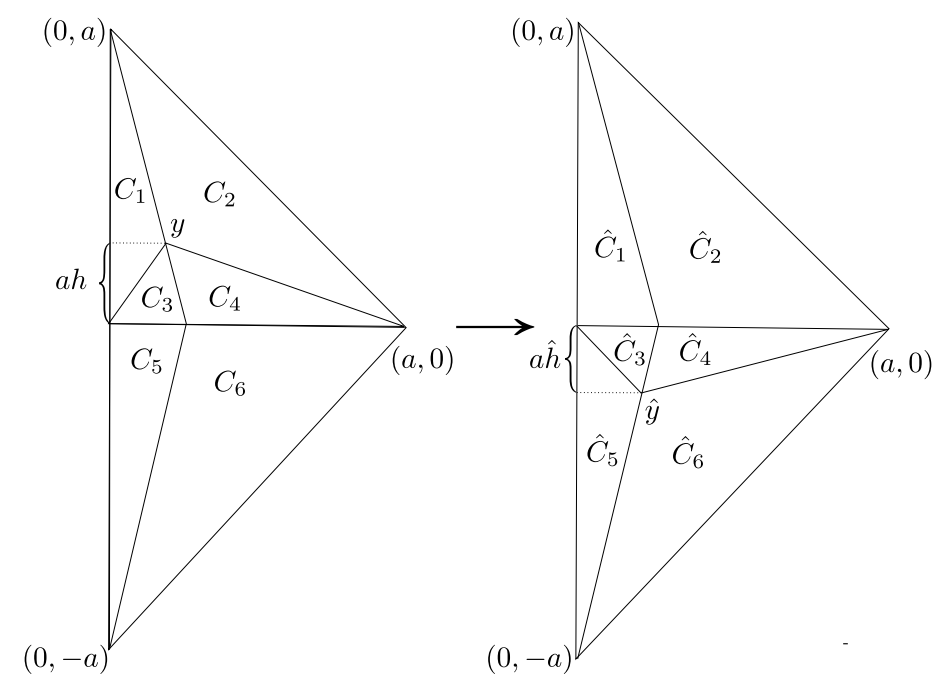

Figure 1. The division of $V$ into triangles and the map $H(h, \epsilon)$.

Note that by Lemma 5.1. $H(h, \epsilon)$ is well defined everywhere on $V$ and also $\left.H(h, \epsilon)\right|_{\partial V}=$ $\left.I d\right|_{\partial V}$. Moreover

$$
\begin{array}{rlr}
\operatorname{lin}\left(\left.H(h, \epsilon)\right|_{C_{1}}\right) & :=\left[\begin{array}{cc}
1+\frac{h}{1-h} & 0 \\
\frac{-h}{\epsilon(1-h)} & 1
\end{array}\right] ; & \operatorname{lin}\left(\left.H(h, \epsilon)\right|_{C_{2}}\right):=\left[\begin{array}{cc}
1-\frac{\epsilon h}{(1-h)(1-\epsilon)} & \frac{-\epsilon h}{(1-h)(1-\epsilon)} \\
\frac{h}{(1-h)(1-\epsilon)} & 1+\frac{h}{(1-h)(1-\epsilon)}
\end{array}\right] ; \\
\operatorname{lin}\left(\left.H(h, \epsilon)\right|_{C_{3}}\right):=\left[\begin{array}{cc}
1-\frac{h}{1+h} & \frac{2 \epsilon}{1+h} \\
\frac{-h}{\epsilon(1+h)} & 1-\frac{2 h}{1+h}
\end{array}\right] ; & \operatorname{lin}\left(\left.H(h, \epsilon)\right|_{C_{4}}\right):=\left[\begin{array}{cc}
1+\frac{\epsilon h}{(1+h)(1-\epsilon)} & \frac{\epsilon(2+h-2 \epsilon)}{(1+h)(1-\epsilon)} \\
\frac{h}{(1+h)(1-\epsilon)} & 1-\frac{h(1-2 \epsilon)}{(1+h)(1-\epsilon)}
\end{array}\right] ; \\
\operatorname{lin}\left(\left.H(h, \epsilon)\right|_{C_{5}}\right) & :=\left[\begin{array}{cc}
1-\frac{h}{1+h} & 0 \\
\frac{-h}{\epsilon(1+h)} & 1
\end{array}\right] ; & \operatorname{lin}\left(\left.H(h, \epsilon)\right|_{C_{6}}\right):=\left[\begin{array}{cc}
1+\frac{\epsilon h}{(1+h)(1-\epsilon)} & \frac{-h \epsilon}{(1+h)(1-\epsilon)} \\
\frac{h}{(1+h)(1-\epsilon)} & 1-\frac{h}{(1+h)(1-\epsilon)}
\end{array}\right] .
\end{array}
$$

By (29) we have

$$
\operatorname{det}\left(\operatorname{lin}\left(\left.H(h, \epsilon)\right|_{C_{1}}\right)\right)=\operatorname{det}\left(\operatorname{lin}\left(\left.H(h, \epsilon)\right|_{C_{2}}\right)\right)=\frac{1}{1-h} \geq 1,
$$

and

$$
\begin{aligned}
& \operatorname{det}\left(\operatorname{lin}\left(\left.H(h, \epsilon)\right|_{C_{3}}\right)\right)=\operatorname{det}\left(\operatorname{lin}\left(\left.H(h, \epsilon)\right|_{C_{4}}\right)\right) \\
& =\operatorname{det}\left(\operatorname{lin}\left(\left.H(h, \epsilon)\right|_{C_{5}}\right)\right)=\operatorname{det}\left(\operatorname{lin}\left(\left.H(h, \epsilon)\right|_{C_{6}}\right)\right)=\frac{1}{1+h} \leq 1 .
\end{aligned}
$$

It is also worth noting that $(0,0)$ is fixed by the affine maps $\left.H(h, \epsilon)\right|_{C_{1}},\left.H(h, \epsilon)\right|_{C_{3}}$ and $\left.H(h, \epsilon)\right|_{C_{5}}$, while $(a, 0)$ is a common fixed point for the transformations $\left.H(h, \epsilon)\right|_{C_{2}},\left.H(h, \epsilon)\right|_{C_{4}}$ and $\left.H(h, \epsilon)\right|_{C_{6}}$.

We can also define $H(h, \epsilon): V \rightarrow V$ for $-1<h \leq 0$. Let $J: \mathbb{R}^{2} \rightarrow \mathbb{R}^{2}$ be the reflection across the $x$-axis. Note that $J V=V, J V_{1}=V_{2}$ and $J V_{2}=V_{1}$. Now define $\hat{h}:=\frac{h}{1+|h|}$, $C_{i}(h, \epsilon):=J\left(C_{i}(-h, \epsilon)\right), \hat{C}_{i}(h, \epsilon):=J\left(\hat{C}_{i}(-h, \epsilon)\right)$ and

$$
H(h, \epsilon):=J \circ H(-h, \epsilon) \circ J .
$$


For $i=1, \ldots, 6$ we have

$$
\operatorname{lin}\left(\left.H(h, \epsilon)\right|_{C_{i}}=J \circ \operatorname{lin}\left(\left.H(-h, \epsilon)\right|_{C_{i}} \circ J .\right.\right.
$$

Since $J$ is an isometry, we also obtain

$$
\operatorname{det}\left(\operatorname{lin}\left(\left.H(h, \epsilon)\right|_{C_{1}}\right)\right)=\operatorname{det}\left(\operatorname{lin}\left(\left.H(h, \epsilon)\right|_{C_{2}}\right)\right)=\frac{1}{1+h} \geq 1,
$$

and

$$
\begin{aligned}
& \operatorname{det}\left(\operatorname{lin}\left(\left.H(h, \epsilon)\right|_{C_{3}}\right)\right)=\operatorname{det}\left(\operatorname{lin}\left(\left.H(h, \epsilon)\right|_{C_{4}}\right)\right) \\
& =\operatorname{det}\left(\operatorname{lin}\left(\left.H(h, \epsilon)\right|_{C_{5}}\right)\right)=\operatorname{det}\left(\operatorname{lin}\left(\left.H(h, \epsilon)\right|_{C_{6}}\right)\right)=\frac{1}{1-h} \leq 1 .
\end{aligned}
$$

Hence in general for $-1<h<1$ we have

$$
\operatorname{det}\left(\operatorname{lin}\left(\left.H(h, \epsilon)\right|_{C_{1}}\right)\right)=\operatorname{det}\left(\operatorname{lin}\left(\left.H(h, \epsilon)\right|_{C_{2}}\right)\right)=\frac{1}{1-|h|} \geq 1,
$$

and

$$
\begin{aligned}
& \operatorname{det}\left(\operatorname{lin}\left(\left.H(h, \epsilon)\right|_{C_{3}}\right)\right)=\operatorname{det}\left(\operatorname{lin}\left(\left.H(h, \epsilon)\right|_{C_{4}}\right)\right) \\
& =\operatorname{det}\left(\operatorname{lin}\left(\left.H(h, \epsilon)\right|_{C_{5}}\right)\right)=\operatorname{det}\left(\operatorname{lin}\left(\left.H(h, \epsilon)\right|_{C_{6}}\right)\right)=\frac{1}{1+|h|} \leq 1 .
\end{aligned}
$$

Lemma 5.2. For any fixed $\epsilon>0$ and for every $h_{1}, h_{2} \in\left(-\frac{1}{2}, \frac{1}{2}\right)$ we have

$$
d_{\mathrm{Hom}}\left(H\left(h_{1}, \epsilon\right), H\left(h_{2}, \epsilon\right)\right) \leq \frac{20 a}{\epsilon}\left|h_{2}-h_{1}\right| .
$$

Proof. We first prove that

$$
\left\|H\left(h_{1}, \epsilon\right)(x)-H\left(h_{2}, \epsilon\right)(x)\right\| \leq \frac{20 a}{\epsilon}\left|h_{2}-h_{1}\right|,
$$

for every $x \in V$. Indeed, assume that $h_{1}$ and $h_{2}$ are non-negative numbers and $h_{1} \geq h_{2}$. Consider the triangle $W_{1}$ with vertices $(0,0), y\left(h_{1}, \epsilon\right), y\left(h_{2}, \epsilon\right)$ and the triangle $W_{2}$ given by the points $(a, 0), y\left(h_{1}, \epsilon\right), y\left(h_{2}, \epsilon\right)$. Assume that $x \in V \backslash\left(W_{1} \cup W_{2}\right)$. Then $x \in C_{i}\left(h_{1}, \epsilon\right) \Leftrightarrow$ $x \in C_{i}\left(h_{2}, \epsilon\right)$. This implies that both $H\left(h_{1}, \epsilon\right)$ and $H\left(h_{2}, \epsilon\right)$ act on $x$ by affine transformations whose linear parts are of the same form. Since for $i=1, \ldots, 6$ the affine maps $\left.H\left(h_{1}, \epsilon\right)\right|_{C_{i}\left(h_{1}, \epsilon\right)}$ and $\left.H\left(h_{2}, \epsilon\right)\right|_{C_{i}\left(h_{2}, \epsilon\right)}$ share a common fixed point,

we get that

$$
H\left(h_{1}, \epsilon\right)(x)-H\left(h_{2}, \epsilon\right)(x)=\operatorname{lin}\left(\left.H\left(h_{1}, \epsilon\right)\right|_{C_{i}\left(h_{1}, \epsilon\right)}\right) x-\operatorname{lin}\left(\left.H\left(h_{2}, \epsilon\right)\right|_{C_{i}\left(h_{2}, \epsilon\right)}\right) x .
$$

By using the formula 28 for each $i=1, \ldots, 6$ we get

$$
\left\|\operatorname{lin}\left(\left.H\left(h_{1}, \epsilon\right)\right|_{C_{i}\left(h_{1}, \epsilon\right)}\right)-\operatorname{lin}\left(\left.H\left(h_{1}, \epsilon\right)\right|_{C_{i}\left(h_{2}, \epsilon\right)}\right)\right\| \leq \frac{10}{\epsilon}\left(h_{1}-h_{2}\right) .
$$

Since the above norm is the operator norm for $H\left(h_{1}, \epsilon\right)-H\left(h_{2}, \epsilon\right)$ (which is a linear transformation), this implies that

$$
\sup _{x \in V \backslash\left(W_{1} \cup W_{2}\right)}\left\|H\left(h_{1}, \epsilon\right)(x)-H\left(h_{2}, \epsilon\right)(x)\right\| \leq \frac{10}{\epsilon}\left(h_{1}-h_{2}\right)\|x\|<\frac{20 a}{\epsilon}\left(h_{1}-h_{2}\right) .
$$

Next note that $W_{1}=C_{3}\left(h_{1}, \epsilon\right) \cap C_{1}\left(h_{2}, \epsilon\right)$ and $W_{2}=C_{4}\left(h_{1}, \epsilon\right) \cap C_{2}\left(h_{2}, \epsilon\right)$. We now prove that for $x \in W_{1} \cup W_{2}$ we also have $\left\|H\left(h_{1}, \epsilon\right)(x)-H\left(h_{1}, \epsilon\right)(x)\right\| \leq \frac{20 a}{\epsilon}\left(h_{1}-h_{2}\right)$. Suppose that $x \in W_{1}$; the proof for $x \in W_{2}$ is analogous. Consider the segment $I_{x} \subset W_{1}$ with endpoints on the segments $\overline{(0,0), y\left(h_{1}, \epsilon\right)}$ and $\overline{(0,0), y\left(h_{2}, \epsilon\right)}$ such that $x \in I_{x}$ and which is parallel to $\overline{y\left(h_{1}, \epsilon\right), y\left(h_{2}, \epsilon\right)}$. Then

$$
\left|I_{x}\right| \leq\left\|y\left(h_{1}, \epsilon\right)-y\left(h_{2}, \epsilon\right)\right\|=a \sqrt{1+\epsilon}\left(h_{1}-h_{2}\right)<2 a\left(h_{1}-h_{2}\right) .
$$


Note that $I_{x}$ divides the intervals $\overline{(0,0), y\left(h_{1}, \epsilon\right)}$ and $\overline{(0,0), y\left(h_{2}, \epsilon\right)}$ with the same ratio. Since affine transformations do not change the ratio of the lengths of collinear segments and

$$
H\left(h_{1}, \epsilon\right)\left(\overline{(0,0), y\left(h_{1}, \epsilon\right)}\right)=H\left(h_{2}, \epsilon\right)\left(\overline{(0,0), y\left(h_{2}, \epsilon\right)}\right)=\overline{(0,0),(0, \epsilon a)},
$$

it follows that the segments $H\left(h_{1}, \epsilon\right)\left(I_{x}\right)$ and $H\left(h_{2}, \epsilon\right)\left(I_{x}\right)$ share a common endpoint in $\overline{(0,0),(0, \epsilon a)}$. Using again the conservation of the ratio of the lengths of collinear segments by affine transformations, we get

$$
\frac{\left|H\left(h_{1}, \epsilon\right)\left(I_{x}\right)\right|}{\left|I_{x}\right|}=\frac{\left|H\left(h_{1}, \epsilon\right)\left(\overline{y\left(h_{1}, \epsilon\right), y\left(h_{2}, \epsilon\right)}\right)\right|}{\left|\overline{y\left(h_{1}, \epsilon\right), y\left(h_{2}, \epsilon\right)}\right|}=\frac{\left|H\left(h_{1}, \epsilon\right)\left(\overline{y\left(h_{1}, \epsilon\right),(\epsilon a, 0)}\right)\right|}{\left|\overline{y\left(h_{1}, \epsilon\right),(\epsilon a, 0)}\right|}=\frac{1}{1+h_{1}} \leq 1,
$$

and

$$
\frac{\left|H\left(h_{2}, \epsilon\right)\left(I_{x}\right)\right|}{\left|I_{x}\right|}=\frac{\left|H\left(h_{2}, \epsilon\right)\left(\overline{y\left(h_{1}, \epsilon\right), y\left(h_{2}, \epsilon\right)}\right)\right|}{\left|\overline{y\left(h_{1}, \epsilon\right), y\left(h_{2}, \epsilon\right)}\right|}=\frac{\left|H\left(h_{2}, \epsilon\right)\left(\overline{(0, a), y\left(h_{2}, \epsilon\right)}\right)\right|}{\left|\overline{(0, a), y\left(h_{2}, \epsilon\right)}\right|}=\frac{1}{1-h_{2}} \leq 2 .
$$

As $H\left(h_{1}, \epsilon\right)(x) \in H\left(h_{1}, \epsilon\right)\left(I_{x}\right)$ and $H\left(h_{2}, \epsilon\right)(x) \in H\left(h_{2}, \epsilon\right)\left(I_{x}\right)$, we obtain

$$
\begin{aligned}
\left\|H\left(h_{1}, \epsilon\right)(x)-H\left(h_{2}, \epsilon\right)(x)\right\| & \leq\left|H\left(h_{1}, \epsilon\right)\left(I_{x}\right)\right|+\left|H\left(h_{2}, \epsilon\right)\left(I_{x}\right)\right| \leq 3\left|I_{x}\right| \\
& <6 a\left(h_{2}-h_{1}\right)<\frac{20}{\epsilon} a\left(h_{1}-h_{2}\right) .
\end{aligned}
$$

By proceeding analogously for $h_{2} \geq h_{1}$ we prove that

$$
\left\|H\left(h_{1}, \epsilon\right)(x)-H\left(h_{2}, \epsilon\right)(x)\right\| \leq \frac{20}{\epsilon} a\left(h_{2}-h_{1}\right) .
$$

The case when $h_{1}$ and $h_{2}$ are non-positive is analogous. To prove the similar inequality when $h_{1}$ and $h_{2}$ are of different sign, let $h_{0}:=0$. Then $H\left(h_{0}, \epsilon\right)=I d$. Using the previous case we show that

and

$$
\left\|H\left(h_{2}, \epsilon\right)(x)-x\right\| \leq \frac{20}{\epsilon} a\left|h_{0}-h_{2}\right|,
$$

$$
\left\|H\left(h_{1}, \epsilon\right)(x)-x\right\| \leq \frac{20}{\epsilon} a\left|h_{1}-h_{0}\right| .
$$

Since $h_{1}, h_{2}$ have different sign, the numbers $h_{0}-h_{2}, h_{1}-h_{0}$ are of the same sign. It follows that

$$
\left\|H\left(h_{1}, \epsilon\right)(x)-H\left(h_{2}, \epsilon\right)(x)\right\| \leq\left\|H\left(h_{1}, \epsilon\right)(x)-x\right\|+\left\|H\left(h_{2}, \epsilon\right)(x)-x\right\| \leq \frac{20}{\epsilon} a\left|h_{2}-h_{1}\right| .
$$

By proceeding as in the proof of 35 and replacing $H\left(h_{i}, \epsilon\right)$ by $H^{-1}\left(h_{i}, \epsilon\right)$ for $i=1,2$, we can prove that for every $x \in V$ we also have

$$
\left\|H^{-1}\left(h_{2}, \epsilon\right)(x)-H^{-1}\left(h_{1}, \epsilon\right)(x)\right\| \leq \frac{20}{\epsilon} a\left|h_{2}-h_{1}\right|,
$$

which concludes the proof of the lemma.

Lemma 5.3. Let $V, V_{1}$ and $V_{2}$ be the triangles defined above. Let $0<\hat{\varepsilon}<10^{-8}$, and let $\kappa>0$. Suppose that $f \in L^{1}(V)$ satisfies

$$
f>\kappa ; \quad \frac{1}{1+\hat{\varepsilon}}<f \text { or } f<\frac{1}{1-\hat{\varepsilon}} ; \quad \int_{V} f(x) d x=\operatorname{Leb}(V) .
$$

Let $\mu_{f}:=f d x$. Then there exists a piecewise affine homeomorphism $H_{f}: V \rightarrow V$ such that

(i) $\left(H_{f}\right)_{*} \mu_{f}\left(V_{i}\right)=\operatorname{Leb}\left(V_{i}\right)$ for $i=1,2$;

(ii) $\left.H_{f}\right|_{\partial V}=\left.I d\right|_{\partial V}$;

(iii) there exists $-\hat{\varepsilon}<h_{f}<\hat{\varepsilon}$ such that $\operatorname{det}\left(D H_{f}^{-1}\right)$ is constant on each $V_{i}$ and is equal to $1 \pm h_{f} ;$

(iv) the Lipschitz constants of $H_{f}$ and $H_{f}^{-1}$ are less than $\frac{5}{4}$;

(v) the maps $f \mapsto H_{f} \in H o m(V)$ and $f \mapsto \operatorname{det}\left(D H_{f}^{-1}\right) \in L^{\infty}(V)$ are continuous on the set of $f \in L^{1}(V)$ satisfying (38) for a given $\kappa$. 
Proof. Since $\mu_{f}$ is an absolutely continuous measure with respect to Leb(, there are no segments of positive measure $\mu_{f}$ in $V$. Hence there exists a unique $-1<h_{f}<1$ and a point $y=$ $y_{f}=\left(\sqrt{\hat{\varepsilon}} a\left(1-\left|h_{f}\right|\right), h_{f} a\right)$ such that the quadrilateral with vertices $\{(0, a),(0,0),(a, 0), y\}$ and the quadrilateral with vertices $\{(0,-a),(0,0),(a, 0), y\}$ have the same measure $\mu_{f}$ equal to $\frac{1}{2} \operatorname{Leb}(V)$.

Consider the triangles $C_{i}=C_{i}^{f}:=C_{i}\left(h_{f}, \sqrt{\hat{\varepsilon}}\right)$ for $i=1, \ldots, 6$. By the definition of $h_{f}$ we have

$$
\mu_{f}\left(C_{1} \cup C_{2}\right)=\mu_{f}\left(C_{3} \cup C_{4} \cup C_{5} \cup C_{6}\right)=\frac{1}{2} \operatorname{Leb}(V) .
$$

We now evaluate the bounds on $h_{f}$. Assume that $f>\frac{1}{1+\hat{\varepsilon}}$. Since $\operatorname{Leb}(V)=a^{2}$ we have

$$
\begin{aligned}
\frac{1}{2} a^{2} & =\mu_{f}\left(C_{3} \cup C_{4} \cup C_{5} \cup C_{6}\right)=\int_{C_{3} \cup C_{4} \cup C_{5} \cup C_{6}} f(x) d x \\
& >\frac{1}{1+\hat{\varepsilon}} \operatorname{Leb}\left(C_{3} \cup C_{4} \cup C_{5} \cup C_{6}\right)=\frac{1}{1+\hat{\varepsilon}}\left(\frac{1}{2}\left(a+\left|h_{f}\right| a\right) a\right) .
\end{aligned}
$$

Hence

$$
f>\frac{1}{1+\hat{\varepsilon}} \Rightarrow\left|h_{f}\right|<\hat{\varepsilon} .
$$

Now assume that $f<\frac{1}{1-\hat{\varepsilon}}$. Then we have

$$
\begin{aligned}
\frac{1}{2} a^{2} & =\mu_{f}\left(C_{1} \cup C_{2}\right)=\int_{C_{1} \cup C_{2}} f(x) d x \\
& <\frac{1}{(1-\hat{\varepsilon})} \operatorname{Leb}\left(C_{1} \cup C_{2}\right)=\frac{1}{1-\hat{\varepsilon}}\left(\frac{1}{2}\left(a-\left|h_{f}\right| a\right) a\right) .
\end{aligned}
$$

This shows

$$
f<\frac{1}{1-\hat{\varepsilon}} \Rightarrow\left|h_{f}\right|<\hat{\varepsilon}
$$

Definition of $H_{f}$. Define $H_{f}:=H\left(h_{f}, \sqrt{\hat{\varepsilon}}\right)$, a piecewise affine homeomorphism on $V$. Note that by definition we have $\left.H_{f}\right|_{\partial V}=\left.I d\right|_{\partial V}$. Moreover

$$
\left(H_{f}\right)_{*} \mu_{f}\left(V_{1}\right)=\mu_{f}\left(C_{1} \cup C_{2}\right)=\frac{1}{2} \operatorname{Leb}(V)=\operatorname{Leb}\left(V_{1}\right)
$$

and

$$
\left(H_{f}\right)_{*} \mu_{f}\left(V_{2}\right)=\mu_{f}\left(C_{3} \cup C_{4} \cup C_{5} \cup C_{6}\right)=\frac{1}{2} \operatorname{Leb}(V)=\operatorname{Leb}\left(V_{2}\right) .
$$

Hence $H_{f}$ satisfies points (i) and (ii).

Furthermore, by 32 and 33 we have that

$$
\operatorname{det}\left(\operatorname{lin}\left(\left.H_{f}\right|_{C_{1}}\right)\right)=\operatorname{det}\left(\operatorname{lin}\left(\left.H_{f}\right|_{C_{2}}\right)\right)=\frac{1}{1-\left|h_{f}\right|} \geq 1,
$$

and

$$
\begin{aligned}
& \operatorname{det}\left(\operatorname{lin}\left(\left.H_{f}\right|_{C_{3}}\right)\right)=\operatorname{det}\left(\operatorname{lin}\left(\left.H_{f}\right|_{C_{4}}\right)\right) \\
& \operatorname{det}\left(\operatorname{lin}\left(\left.H_{f}\right|_{C_{5}}\right)\right)=\operatorname{det}\left(\operatorname{lin}\left(\left.H_{f}\right|_{C_{6}}\right)\right)=\frac{1}{1+\left|h_{f}\right|} \leq 1 .
\end{aligned}
$$

Note that $V_{1}=\hat{C}_{1} \cup \hat{C}_{2}$ and $V_{2}=\hat{C}_{3} \cup \hat{C}_{4} \cup \hat{C}_{5} \cup \hat{C}_{6}$ for $h_{f} \geq 0$ and $V_{1}=\hat{C}_{3} \cup \hat{C}_{4} \cup \hat{C}_{5} \cup \hat{C}_{6}$ and $V_{2}=\hat{C}_{1} \cup \hat{C}_{2}$ for $h_{f} \leq 0$. Hence by 41) and 42 we have

$$
\operatorname{det}\left(\operatorname{lin}\left(\left.H_{f}^{-1}\right|_{V_{1}}\right)\right)=1-h_{f} \quad \text { and } \quad \operatorname{det}\left(\operatorname{lin}\left(\left.H_{f}^{-1}\right|_{V_{2}}\right)\right)=1+h_{f}
$$

Thus $H_{f}$ satisfies (iii). 
The norm of the linear part. We will now prove that $\left.\|\left.\operatorname{lin}\left(H_{f}\right)\right|_{C_{i}}\right) \|<\frac{5}{4}$ for $i=1, \ldots, 6$. Note that each of the matrices $\left.\operatorname{lin}\left(H_{f}\right)\right|_{C_{i}}$ is of the form $M=\left(\begin{array}{cc}1+b & c \\ d & 1+e\end{array}\right)$, where $|b|,|c|,|d|,|e|<$ $3 \sqrt{\hat{\varepsilon}}$. Hence, using the formula $(28)$ and the fact that $\varepsilon<10^{-8}$, we obtain that

$$
\begin{aligned}
\left\|\left.\operatorname{lin}\left(H_{f}\right)\right|_{C_{i}}\right\|< & \\
& <\sqrt{\frac{2(1+3 \sqrt{\hat{\varepsilon}})+2 \cdot 3 \sqrt{\hat{\varepsilon}}+\sqrt{(2(1+3 \sqrt{\hat{\varepsilon}})+2 \cdot 3 \sqrt{\hat{\varepsilon}})^{2}-4\left(\operatorname{det}\left(\left.\operatorname{lin}\left(H_{f}\right)\right|_{\hat{C}_{i}}\right)\right)^{2}}}{2}} \\
& <\sqrt{\frac{2+12 \sqrt{\hat{\varepsilon}}+36 \hat{\varepsilon}+\sqrt{(2+12 \sqrt{\hat{\varepsilon}}+36 \hat{\varepsilon})^{2}-4\left(\frac{1}{1+\hat{\varepsilon}}\right)^{2}}}{2}} \\
& <\sqrt{1+5 \sqrt[4]{\hat{\varepsilon}}}<\frac{5}{4} .
\end{aligned}
$$

In the same way we prove that $\left.\|\left.\operatorname{lin}\left(H_{f}\right)^{-1}\right|_{\hat{C}_{i}}\right) \|<\frac{5}{4}$. Thus $H_{f}$ satisfies (iv).

Continuity of $f \mapsto H_{f}$. Suppose that $f, g \in L^{1}(V)$ satisfy (38). By Lemma 5.2, we already know that

$$
d_{\mathrm{Hom}}\left(H_{f}, H_{g}\right) \leq \frac{20}{\sqrt{\hat{\varepsilon}}} a\left|h_{f}-h_{g}\right|
$$

We prove that

$$
\left|h_{f}-h_{g}\right| \leq C\|f-g\|_{L^{1}},
$$

for some constant $C>0$ depending only on $a$ and $\kappa$. Let $\delta:=\|f-g\|_{L^{1}}$.

Case $h_{f}$ and $h_{g}$ have the same sign. Assume that $h_{f} \geq h_{g} \geq 0$ or $0 \geq h_{g} \geq h_{f}$. Then

$$
\begin{aligned}
0 & =\mu_{f}\left(C_{1}^{f} \cup C_{2}^{f}\right)-\mu_{g}\left(C_{1}^{g} \cup C_{2}^{g}\right) \\
& =\int_{C_{1}^{f} \cup C_{2}^{f}} f(x) d x-\int_{C_{1}^{g} \cup C_{2}^{g}} g(x) d x \\
& =\int_{C_{1}^{g} \cup C_{2}^{g}}(f-g)(x) d x-\int_{\left(C_{1}^{g} \cup C_{2}^{g}\right) \backslash\left(C_{1}^{f} \cup C_{2}^{f}\right)} g(x) d x \\
& \leq \delta-\left|h_{f}-h_{g}\right| \frac{a \kappa}{2},
\end{aligned}
$$

and hence

$$
\left|h_{f}-h_{g}\right| \leq \frac{2 \delta}{a \kappa}
$$

Thus 46 holds with $C=\frac{2}{a \kappa}$.

Case of $h_{f}, h_{g}$ with different sign. Suppose that $h_{f} \geq 0 \geq h_{g}$. Then we have

$$
\begin{aligned}
0 & =\mu_{f}\left(C_{1}^{f} \cup C_{2}^{f}\right)-\mu_{g}\left(C_{3}^{g} \cup C_{4}^{g} \cup C_{5}^{g} \cup C_{6}^{g}\right) \\
& =\int_{C_{1}^{f} \cup C_{2}^{f}} f(x) d x-\int_{C_{3}^{g} \cup C_{4}^{g} \cup C_{5}^{g} \cup C_{6}^{g}} g(x) d x \\
& =\int_{C_{1}^{f} \cup C_{2}^{f}}(f-g)(x) d x-\int_{\left(C_{3}^{g} \cup C_{4}^{g} \cup C_{5}^{g} \cup C_{6}^{g}\right) \backslash\left(C_{1}^{f} \cup C_{2}^{f}\right)} g(x) d x \\
& \leq \delta-\left|h_{f}-h_{g}\right| \frac{a \kappa}{2} .
\end{aligned}
$$

Thus we have

which completes the proof of 46 .

$$
0 \leq h_{f}-h_{g} \leq \frac{2 \delta}{a \kappa}
$$

By combining (45) and (46) we obtain

$$
d_{H o m}\left(H_{f}, H_{g}\right) \leq \frac{10}{\sqrt{\hat{\varepsilon}}}\left|h_{f}-h_{g}\right|<\frac{10}{\sqrt{\hat{\varepsilon}}} C\|f-g\|_{L^{1}} .
$$


This concludes the proof of the continuity of $f \mapsto H_{f}$. By the formula given in (43), the continuity of the map $f \mapsto h_{f}$ also implies the continuity of the map $f \mapsto \operatorname{det}\left(D H_{f}^{-1}\right) \in L^{\infty}(V)$. Thus (v) is proved.

Let $(X, \mu)$ be a standard metric probability space. For $0<s_{1}<s_{2}$, define $\mathcal{W}\left(X, s_{1}, s_{2}\right) \subset$ $L^{1}(X, \mu)$ by

$$
\mathcal{W}\left(X, s_{1}, s_{2}\right):=\left\{f \in L^{1}(X) ; s_{1}<f<s_{2} ; \int_{X} f d \mu(x)=\mu(X)\right\} .
$$

Let $V$ be the triangle with vertices $(0,-a),(0, a),(a, 0)$, equipped with the (normalized) 2dimensional Lebesgue measure. We need the following lemma.

Lemma 5.4. Let $H: \mathcal{W}\left(V, s_{1}, s_{2}\right) \rightarrow \operatorname{Hom}(V)$ be a continuous map.Assume that there exists $\ell>0$ such that, for every $f \in \mathcal{W}\left(V, s_{1}, s_{2}\right)$, the homeomorphism $H(f)^{-1}$ is Lipschitz with constant $\ell$. Then the transformation

$$
W\left(s_{1}, s_{2}\right) \ni f \mapsto f \circ H(f) \in L^{1}(V)
$$

is continuous.

Proof. Take $f \in \mathcal{W}\left(V, s_{1}, s_{2}\right)$ and $\epsilon>0$. Let $f_{\epsilon}: V \rightarrow \mathbb{R}$ be a uniformly continuous function such that $\left\|f_{\epsilon}-f\right\|_{L^{1}}<\epsilon$. Let $0<\delta<\epsilon$ be such that

$$
\|x-y\|<\delta \Rightarrow\left|f_{\epsilon}(x)-f_{\epsilon}(y)\right|<\epsilon .
$$

Consider $0<\delta^{\prime}<\epsilon$ such that for every $g \in W\left(V, s_{1}, s_{2}\right)$ we have

$$
\|f-g\|_{L^{1}}<\delta^{\prime} \Rightarrow d_{\text {Hom }}(H(f), H(g))<\delta,
$$

and let $g \in W\left(V, s_{1}, s_{2}\right)$ be such that $\|f-g\|_{L^{1}}<\delta^{\prime}$. Since $H(g)^{-1}$ is Lipschitz with constant $\ell, H(g)_{*} L e b$ is an absolutely continuous measure with density bounded by $\ell^{2}$. Hence for every $h \in L^{1}(V)$ we have

$$
\|h \circ H(g)\|_{L^{1}}=\int_{V}|h \circ H(g)(x)| d x \leq \int_{V} \ell^{2}|h(x)| d x=\ell^{2}\|h\|_{L^{1}} .
$$

Then

$$
\|f \circ H(f)-g \circ H(g)\|_{L^{1}} \leq\|f \circ H(f)-f \circ H(g)\|_{L^{1}}+\|f \circ H(g)-g \circ H(g)\|_{L^{1}}
$$

and, by 50 ,

Moreover

$$
\|f \circ H(g)-g \circ H(g)\|_{L^{1}} \leq \ell^{2}\|f-g\|_{L^{1}} .
$$

$$
\begin{aligned}
\|f \circ H(f)-f \circ H(g)\|_{L^{1}} \leq & \left\|f \circ H(f)-f_{\epsilon} \circ H(f)\right\|_{L^{1}}+\left\|f_{\epsilon} \circ H(f)-f_{\epsilon} \circ H(g)\right\|_{L^{1}} \\
& +\left\|f_{\epsilon} \circ H(g)-f \circ H(g)\right\|_{L^{1}} \\
\leq & 2 \ell^{2}\left\|f-f_{\epsilon}\right\|_{L^{1}}+\left\|f_{\epsilon} \circ H(f)-f_{\epsilon} \circ H(g)\right\|_{L^{1}},
\end{aligned}
$$

where the last inequality comes from $(50)$. By 49 and 48, , we have

$$
\left\|f_{\epsilon} \circ H(f)-f_{\epsilon} \circ H(g)\right\|_{L^{1}}<\epsilon .
$$

To sum up we obtain

$$
\|f \circ H(f)-g \circ H(g)\|_{L^{1}} \leq \ell^{2}\|f-g\|_{L^{1}}+2 \ell^{2}\left\|f-f_{\epsilon}\right\|_{L^{1}}+\epsilon \leq\left(3 \ell^{2}+1\right) \epsilon,
$$

which proves the assertion.

Remark 5.5. The statement of Lemma 5.4 remains valid if we replace $V$ with any 2-dimensional Riemannian surface $M$

Lemma 5.6. Let $0<\hat{\varepsilon}<10^{-8}$. Let $f \in \mathcal{W}\left(V, \frac{1}{1+\hat{\varepsilon}}, \frac{1}{1-\hat{\varepsilon}}\right)$ and $\mu_{f}:=f d x$. Then there exists a homeomorphism $H_{f}: V \rightarrow V$, depending continuously on $f$, such that $\left(H_{f}\right)_{*} \mu_{f}=$ Leb and $\left.H_{f}\right|_{\partial V}=\left.I d\right|_{\partial V}$. 
Proof. We assume that $a=1$. The prove for $a \neq 1$ goes along the same lines. Let $f \in$ $\mathcal{W}\left(V, \frac{1}{1+\hat{\varepsilon}}, \frac{1}{1-\hat{\varepsilon}}\right)$. Denote by $V_{1}^{1}$ and $V_{2}^{1}$ the two halves of $V$ which are both isosceles right triangles with $\operatorname{diam}\left(V_{1}^{1}\right)=\operatorname{diam}\left(V_{2}^{1}\right)=\sqrt{2}$.

Inductively, for $n \in \mathbb{N}$ define the family $\left\{V_{i}^{n}\right\}_{i=1}^{2^{n}}$ of congruent right isosceles triangles which divide $V, V_{i}^{n}=V_{2 i-1}^{n+1} \cup V_{2 i}^{n+1}$ for $i=1, \ldots, 2^{n}$ and they satisfy

$$
\operatorname{diam}\left(V_{n}^{i}\right)=\frac{1}{\sqrt{2}^{n-2}}
$$

We will construct $H_{f}$ inductively as a limit of piecewise affine transformations.

In the first step, using Lemma 5.3 we obtain a piecewise affine homeomorphism $H_{f}^{1}: V \rightarrow V$ such that

$$
\left(H_{f}^{1}\right)_{*} \mu_{f}\left(V_{1}^{1}\right)=\left(H_{f}^{1}\right)_{*} \mu_{f}\left(V_{2}^{1}\right)=\frac{1}{2} \operatorname{Leb}(V) \text { and }\left.H_{f}^{1}\right|_{\partial V}=\left.I d\right|_{\partial V} .
$$

Moreover $\operatorname{det}\left(D\left(H_{f}^{1}\right)^{-1}\right)$ is constant on each $V_{1}^{1}$ and $V_{2}^{1}$.

Suppose now that for some $n \in \mathbb{N}$ we have constructed piecewise affine homeomorphisms $H_{f}^{j}: V \rightarrow V$ for $j=1, \ldots, n$ such that for all $i=1, \ldots, 2^{n}$ we have

$$
\left(H_{f}^{n} \circ \ldots \circ H_{f}^{1}\right)_{*} \mu_{f}\left(V_{i}^{n}\right)=\frac{1}{2^{n}} \operatorname{Leb}(V)=\operatorname{Leb}\left(V_{i}^{n}\right) \text { and }\left.H_{f}^{j}\right|_{\partial V}=\left.I d\right|_{\partial V} .
$$

Moreover, suppose that $\operatorname{det}\left(D\left(H_{f}^{n} \circ \ldots \circ H_{f}^{1}\right)^{-1}\right)$ is constant on each $V_{i}^{n}$ and equals $d_{i}^{n}>0$.

With these assumptions the measure $\left(H_{f}^{n} \circ \ldots \circ H_{f}^{1}\right)_{*} \mu_{f}$ is absolutely continuous and its density $f_{n}: V \rightarrow \mathbb{R}_{>0}$ satisfies

$$
f_{n}(x)=d_{i}^{n} \cdot f\left(\left(H_{f}^{n} \circ \ldots \circ H_{f}^{1}\right)^{-1} x\right) \text { if } x \in V_{i}^{n},
$$

and by 52

$$
\int_{V_{i}^{n}} f_{n}(x) d x=\left(H_{f}^{n} \circ \ldots \circ H_{f}^{1}\right)_{*} \mu_{f}\left(V_{i}^{n}\right)=\operatorname{Leb}\left(V_{i}^{n}\right) .
$$

Take any $1 \leq i \leq 2^{n}$. In view of 47), if $d_{i}^{n}<1$ then

$$
f_{n}(x)<\frac{d_{i}^{n}}{1-\hat{\varepsilon}}<\frac{1}{1-\hat{\varepsilon}} \text { for all } x \in V_{i}^{n}
$$

and if $d_{i}^{n} \geq 1$ then

$$
f_{n}(x)>\frac{d_{i}^{n}}{1+\hat{\varepsilon}} \geq \frac{1}{1+\hat{\varepsilon}} \text { for all } x \in V_{i}^{n}
$$

It follows that $f_{n}: V_{i}^{n} \rightarrow \mathbb{R}_{>0}$ is a positive density satisfying 38 with $\kappa=\frac{d_{i}^{n}}{1+\hat{\varepsilon}}$. Hence we can apply Lemma 5.3 to the triangle $V_{i}^{n}$ and the density function $f_{n}: V_{i}^{n} \rightarrow \mathbb{R}_{>0}$, thus obtaining a piecewise affine homeomorphism $H_{f}^{n+1, i}: V_{i}^{n} \rightarrow V_{i}^{n}$ such that

$$
\begin{gathered}
\left(H_{f}^{n+1, i}\right)_{*}\left(\left.\mu_{f_{n}}\right|_{V_{i}^{n}}\right)\left(V_{2 i-1}^{n+1}\right)=\left(H_{f}^{n+1, i}\right)_{*}\left(\left.\mu_{f_{n}}\right|_{V_{i}^{n}}\right)\left(V_{2 i}^{n+1}\right)=\frac{1}{2} \operatorname{Leb}\left(V_{i}^{n}\right)=\frac{1}{2^{n+1}} \operatorname{Leb}(V), \\
\left.H_{f}^{n+1, i}\right|_{\partial V_{i}^{n}}=\left.I d\right|_{\partial V_{i}^{n}},
\end{gathered}
$$

and

$$
\operatorname{det} D\left(\left(H_{f}^{n+1, i}\right)^{-1}\right) \text { is constant on both } V_{2 i-1}^{n+1} \text { and } V_{2 i}^{n+1} \text {. }
$$

Finally we define a piecewise affine homeomorphism $H_{f}^{n+1}: V \rightarrow V$ given by

$$
H_{f}^{n+1}(x):=H_{f}^{n+1, i}(x) \text { whenever } x \in V_{i}^{n} .
$$

Then $H_{f}^{n+1}\left(V_{i}^{n}\right)=V_{i}^{n}$ and, by (54), we have $\left.H_{f}^{n+1}\right|_{\partial V}=\left.I d\right|_{\partial V}$. Moreover, by (53),

$$
\left(H_{f}^{n+1} \circ \ldots \circ H_{f}^{1}\right)_{*} \mu_{f}\left(V_{2 i-1}^{n+1}\right)=\left(H_{f}^{n+1} \circ \ldots \circ H_{f}^{1}\right)_{*} \mu_{f}\left(V_{2 i}^{n+1}\right)=\frac{1}{2^{n+1}} \operatorname{Leb}(V) .
$$


In view of $55, \operatorname{det}\left(D\left(H_{f}^{n+1}\right)^{-1}\right)$ is constant on each $V_{j}^{n+1}$ for $j=1, \ldots, 2^{n+1}$ and then so is $\operatorname{det}\left(D\left(H_{f}^{n+1} \circ \ldots \circ H_{f}^{1}\right)^{-1}\right)$. Thus, we have proved that $H_{f}^{n+1}$ satisfies the assumptions of the induction.

Note that, by (iii) in Lemma 5.3 , we have

$$
1-\hat{\varepsilon}<\operatorname{det}\left(D\left(H_{f}^{n}\right)^{-1}\right)<1+\hat{\varepsilon} \text { for every } n \in \mathbb{N},
$$

and since $\left(H_{f}^{j}\right)^{-1}$ are piecewise linear homeomorphisms, it follows that

$$
(1-\hat{\varepsilon})^{n} \leq \operatorname{det}\left(D\left(H_{f}^{n} \circ \ldots \circ H_{f}^{1}\right)^{-1}\right) \leq(1+\hat{\varepsilon})^{n} \text { almost everywhere }
$$

and the above inequalities do not depend on $f$.

We now show that

$$
H_{f}(x):=\lim _{n \rightarrow \infty} H_{f}^{n} \circ \ldots \circ H_{f}^{1}(x)
$$

yields a homeomorphism $H_{f}: V \rightarrow V$. First note that

$$
H_{f}^{m}\left(V_{i}^{n}\right)=V_{i}^{n} \text { for } i=1, \ldots, 2^{n} \text { and } m>n .
$$

Moreover, by 51 we have

$$
\max _{i=1, \ldots, 2^{n}} \operatorname{diam}\left(V_{i}^{n}\right) \rightarrow 0 \text { for } n \rightarrow \infty
$$

This implies that $\left\{H_{f}^{m} \circ \ldots \circ H_{f}^{1}\right\}_{n \in \mathbb{N}}$ is a Cauchy sequence. Indeed, for any $\epsilon>0$ by 60 we can choose $N \in \mathbb{N}$ such that $\max _{i=1, \ldots, 2^{N}} \operatorname{diam}\left(V_{i}^{N}\right)<\epsilon$. Moreover, by (59), for all $m, n \geq N$ we have

$$
H_{f}^{n} \circ \ldots \circ H_{f}^{1}(x) \in V_{i}^{N} \Longleftrightarrow H_{f}^{m} \circ \ldots \circ H_{f}^{1}(x) \in V_{i}^{N} .
$$

Hence $\left\|H_{f}^{n} \circ \ldots \circ H_{f}^{1}(x)-H_{f}^{m} \circ \ldots \circ H_{f}^{1}(x)\right\|<\epsilon$ for all $x \in V$. It follows that the map $H_{f}: V \rightarrow V$ given by (58) is well defined and the convergence in (58) is uniform. This implies that $H_{f}$ is continuous. Since $\left.H_{f}^{n}\right|_{\partial V}=\left.I d\right|_{\partial V}$ for all $n \in \mathbb{N}$, we also have $\left.H_{f}\right|_{\partial V}=\left.I d\right|_{\partial V}$.

Set $W_{i}^{n}:=\left(H_{f}^{n} \circ \ldots \circ H_{f}^{1}\right)^{-1}\left(V_{i}^{n}\right)$. In view of $(59)$,

$$
W_{i}^{n}=\left(H_{f}^{m} \circ \ldots \circ H_{f}^{1}\right)^{-1}\left(V_{i}^{n}\right) \text { for } m>n .
$$

Therefore,

$$
\left(H_{f}^{n} \circ \ldots \circ H_{f}^{1}\right)^{-1}(x) \in W_{i}^{N} \Longleftrightarrow\left(H_{f}^{m} \circ \ldots \circ H_{f}^{1}\right)^{-1}(x) \in W_{i}^{N} \text { if } m, n \geq N .
$$

By (iv) in Lemma $5.3\left(H_{f}^{n}\right)^{-1}$ is a Lipschitz automorphism with a Lipschitz constant $\frac{5}{4}$. Thus, by (51), we have

so

$$
\operatorname{diam}\left(W_{i}^{n}\right)<\operatorname{diam}\left(V_{i}^{n}\right)\left(\frac{5}{4}\right)^{n}=2\left(\frac{5}{4 \sqrt{2}}\right)^{n}
$$

$$
\max _{i=1, \ldots, 2^{n}} \operatorname{diam}\left(W_{i}^{n}\right) \rightarrow 0 \text { for } n \rightarrow \infty .
$$

Using (63) and 62 and repeating the same arguments as for $H_{f}$ by replacing $V_{i}^{n}$ with $W_{i}^{n}$, we obtain that the map $G_{f}: V \rightarrow V$ given by

$$
G_{f}(x):=\lim _{n \rightarrow \infty}\left(H_{f}^{n} \circ \ldots \circ H_{f}^{1}\right)^{-1}(x)
$$

is well defined and continuous. We now show that $H_{f} \circ G_{f}=I d$ and $G_{f} \circ H_{f}=I d$. First note that in view of (61) and the compactness of $V_{i}^{n}$ and $W_{i}^{n}$ we have $H_{f}\left(W_{i}^{n}\right)=V_{i}^{n}$ and $G_{f}\left(V_{i}^{n}\right)=W_{i}^{n}$. Hence $H_{f} \circ G_{f}\left(V_{i}^{n}\right)=V_{i}^{n}$ and $G_{f} \circ H_{f}\left(W_{i}^{n}\right)=W_{i}^{n}$. Let $\epsilon>0$ and $N \in \mathbb{N}$ be such that

This implies that

$$
\max _{i=1, \ldots, 2^{N}} \operatorname{diam}\left(V_{i}^{N}\right)<\epsilon \text { and } \max _{i=1, \ldots, 2^{N}} \operatorname{diam}\left(W_{i}^{N}\right)<\epsilon .
$$

$$
\left\|H_{f}\left(G_{f}(x)\right)-x\right\|<\epsilon \text { and }\left\|G_{f}\left(H_{f}(x)\right)-x\right\|<\epsilon \text { for every } x \in V .
$$


Since $\epsilon$ was arbitrary, this shows that $H_{f} \circ G_{f}=I d$ and $G_{f} \circ H_{f}=I d$. Thus $H_{f}$ is a homeomorphism.

Note that the family of sets $\left\{V_{i}^{n} ; n \in \mathbb{N}, i=1, \ldots, 2^{n}\right\}$ generates the Borel $\sigma$-algebra on $\mathrm{V}$. Since $\left(H_{f}\right)^{-1}\left(V_{i}^{n}\right)=W_{i}^{n}=\left(H_{f}^{n} \circ \ldots \circ H_{f}^{1}\right)^{-1}\left(V_{i}^{n}\right)$, by (52), we have

$$
\left(H_{f}\right)_{*} \mu_{f}\left(V_{i}^{n}\right)=\left(H_{f}^{n} \circ \ldots \circ H_{f}^{1}\right)_{*} \mu_{f}\left(V_{i}^{n}\right)=\operatorname{Leb}\left(V_{i}^{n}\right) .
$$

It follows that $\left(H_{f}\right)_{*} \mu_{f}=L e b$.

In the reminder of the proof we will show that $H_{f}$ depends continuously on $f$. Fix $\epsilon>0$ and then choose $m \in \mathbb{N}$ such that $2(5 / 4 \sqrt{2})^{m}<\epsilon / 3$. Then

$$
\max _{i=1, \ldots, 2^{m}} \operatorname{diam}\left(V_{i}^{m}\right)<\frac{\epsilon}{3} \text { and } \max _{i=1, \ldots, 2^{m}} \operatorname{diam}\left(W_{i}^{m}\right)<\frac{\epsilon}{3} .
$$

Since $\left(H_{f}\right)^{-1}\left(V_{i}^{m}\right)=W_{i}^{m}=\left(H_{f}^{m} \circ \ldots \circ H_{f}^{1}\right)^{-1}\left(V_{i}^{m}\right)$, it follows that

$$
\sup _{x \in V}\left\|H_{f}(x)-H_{f}^{m} \circ \ldots \circ H_{f}^{1}(x)\right\|<\frac{\epsilon}{3} \text { and } \sup _{x \in V}\left\|\left(H_{f}\right)^{-1}(x)-\left(H_{f}^{m} \circ \ldots \circ H_{f}^{1}\right)^{-1}(x)\right\|<\frac{\epsilon}{3} \text {. }
$$

Therefore

$$
d_{H o m}\left(H_{f}, H_{f}^{m} \circ \ldots \circ H_{f}^{1}\right)<\frac{\epsilon}{3} \text { for every } f \in \mathcal{W}\left(V, \frac{1}{1+\hat{\varepsilon}}, \frac{1}{1-\hat{\varepsilon}}\right) .
$$

By (v) in Lemma 5.3 the maps $f \mapsto H_{f}^{1} \in \operatorname{Hom}(V)$ and $f \mapsto \operatorname{det} D\left(H_{f}^{1}\right)^{-1} \in L^{\infty}(V)$ are continuous. Suppose now that for $k \geq 1$ we proved that

$$
f \mapsto H_{f}^{k} \circ \ldots \circ H_{f}^{1} \in H o m(V) \text { and } f \mapsto \operatorname{det} D\left(H_{f}^{k} \circ \ldots \circ H_{f}^{1}\right)^{-1} \in L^{\infty}(V)
$$

are continuous. We now prove that

$$
f \mapsto H_{f}^{k+1} \circ \ldots \circ H_{f}^{1} \text { and } f \mapsto \operatorname{det} D\left(H_{f}^{k+1} \circ \ldots \circ H_{f}^{1}\right)^{-1}
$$

are also continuous. Since for every $i=1, \ldots, k, H_{f}^{i}$ and $\left(H_{f}^{i}\right)^{-1}$ are Lipschitz homeomorphisms with constant $\frac{5}{4}$, we get

$$
H_{f}^{k} \circ \ldots H_{f}^{1} \text { and }\left(H_{f}^{k} \circ \ldots H_{f}^{1}\right)^{-1} \text { are Lipschitz with constant }\left(\frac{5}{4}\right)^{k} .
$$

Moreover by (57) we have

$$
f_{k}=\operatorname{det} D\left(H_{f}^{k} \circ \ldots \circ H_{f}^{1}\right)^{-1} \cdot\left(f \circ\left(H_{f}^{k} \circ \ldots \circ H_{f}^{1}\right)^{-1}\right) \in \mathcal{W}\left(V, \frac{(1-\hat{\varepsilon})^{k}}{1+\hat{\varepsilon}}, \frac{(1+\hat{\varepsilon})^{k}}{1-\hat{\varepsilon}}\right) .
$$

In view of Lemma 5.3. $H_{f}^{k+1}$ depends continuously on $f_{k}$. By 65 and (66), Lemma 5.4 implies that $f \mapsto f \circ\left(H_{f}^{k} \circ \ldots \circ H_{f}^{1}\right)^{-1} \in L^{1}(V)$ is continuous. Together with 65 this gives the continuity of

$$
f \mapsto f_{k}=f \circ\left(H_{f}^{k} \circ \ldots \circ H_{f}^{1}\right)^{-1} \cdot \operatorname{det} D\left(H_{f}^{k} \circ \ldots \circ H_{f}^{1}\right)^{-1} \in L^{1}(V) .
$$

It follows that $H_{f}^{k+1}$ depends continuously on $f$.

Again, since $H_{f}^{i}$ and $\left(H_{f}^{i}\right)^{-1}$ are Lipschitz with constant $\frac{5}{4}$, for any $x \in V$ and $f, g \in$ $\mathcal{W}\left(V, \frac{1}{1+\hat{\varepsilon}}, \frac{1}{1-\hat{\varepsilon}}\right)$ we have

$$
\begin{aligned}
\| H_{f}^{k+1}( & \left.H_{f}^{k} \circ \ldots \circ H_{f}^{1}(x)\right)-H_{g}^{k+1}\left(H_{g}^{k} \circ \ldots \circ H_{g}^{1}(x)\right) \| \\
\leq & \left\|H_{f}^{k+1}\left(H_{f}^{k} \circ \ldots \circ H_{f}^{1}(x)\right)-H_{g}^{k+1}\left(H_{f}^{k} \circ \ldots \circ H_{f}^{1}(x)\right)\right\| \\
& \quad+\left\|H_{g}^{k+1}\left(H_{f}^{k} \circ \ldots \circ H_{f}^{1}(x)\right)-H_{g}^{k+1}\left(H_{g}^{k} \circ \ldots \circ H_{g}^{1}(x)\right)\right\| \\
\leq & d_{H o m}\left(H_{f}^{k+1}, H_{g}^{k+1}\right)+\frac{5}{4} d_{H \circ m}\left(H_{f}^{k} \circ \ldots \circ H_{f}^{1}, H_{g}^{k} \circ \ldots \circ H_{g}^{1}\right)
\end{aligned}
$$

and similarly

$$
\begin{aligned}
& \left\|\left(H_{f}^{k} \circ \ldots \circ H_{f}^{1}\right)^{-1}\left(H_{f}^{k+1}\right)^{-1}(x)-\left(H_{g}^{k} \circ \ldots \circ H_{g}^{1}\right)^{-1}\left(H_{g}^{k+1}\right)^{-1}(x)\right\| \\
& \quad \leq\left(\frac{5}{4}\right)^{k} d_{H o m}\left(H_{f}^{k+1}, H_{g}^{k+1}\right)+d_{H \circ m}\left(H_{f}^{k} \circ \ldots \circ H_{f}^{1},\left(H_{g}^{k} \circ \ldots \circ H_{g}^{1}\right)\right) .
\end{aligned}
$$


This proves the continuous dependence of $H_{f}^{k+1} \circ \ldots \circ H_{f}^{1}$ on $f$. Finally, since $H_{f}^{i}$ are piecewise linear homeomorphisms, we have

$$
D\left(H_{f}^{k+1} \circ \ldots \circ H_{f}^{1}\right)^{-1}=D\left(H_{f}^{k+1}\right)^{-1} D\left(H_{f}^{k} \circ \ldots \circ H_{f}^{1}\right)^{-1} \text { almost everywhere. }
$$

By (v) in Lemma 5.3. $f_{k} \mapsto \operatorname{det} D\left(H_{f}^{k+1}\right)^{-1} \in L^{\infty}(V)$ depends continuously on $f_{k}$. Since $f \mapsto f_{k} \in L^{1}(V)$ is continuous, it follows that $f \mapsto \operatorname{det} D\left(H_{f}^{k+1}\right)^{-1} \in L^{\infty}(V)$ is also continuous. The uniform boundaries in (56) and in (57), together with (65), yield the continuity of $f \mapsto$ $\operatorname{det} D\left(H_{f}^{k+1} \circ \ldots \circ H_{f}^{1}\right)^{-1} \in L^{\infty}(V)$.

Fix any $f \in \mathcal{W}\left(V, \frac{1}{1+\hat{\varepsilon}}, \frac{1}{1-\hat{\varepsilon}}\right)$. Then there exists $\delta>0$ such that for any $g \in \mathcal{W}\left(V, \frac{1}{1+\hat{\varepsilon}}, \frac{1}{1-\hat{\varepsilon}}\right)$ with $\|f-g\|_{L^{1}}<\delta$ we have

$$
d_{H o m}\left(H_{f}^{m} \circ \ldots \circ H_{f}^{1}, H_{g}^{m} \circ \ldots \circ H_{g}^{1}\right)<\frac{\epsilon}{3} .
$$

Combining this with 64 we obtain

$$
d_{H o m}\left(H_{f}, H_{g}\right)<\epsilon,
$$

which concludes the proof of the continuity of $f \mapsto H_{f}$.

Remark 5.7. Note that the above lemma is also valid for any triangle, since every two triangles are conjugated by an affine map (although the restriction on $\hat{\varepsilon}$ may vary).

The following theorem is the main result of this section.

Theorem 5.8. Let $(M, \Sigma, \zeta)$ be a translation surface. There exists $\varepsilon_{\zeta}=\varepsilon>0$ such that, for all $f \in \mathcal{W}\left(M, \frac{1}{1+\varepsilon}, \frac{1}{1-\varepsilon}\right)$, there exists a homeomorphism $\mathcal{H}_{f}:(M, \Sigma) \rightarrow(M, \Sigma)$ such that $\left(\mathcal{H}_{f}\right)_{*} \mu_{f}=\lambda_{\zeta}$, where $\mu_{f}:=f \lambda_{\zeta}$. Moreover, $\mathcal{H}_{f}$ depends continuously on $f$.

Proof. On $(M, \Sigma)$ consider a triangulation of $m+1$ triangles such that the set of vertices of this triangulation contains the set $\Sigma$. By connectedness, there is an ordering $\left\{U_{i}: 0 \leq i \leq m\right\}$ of the triangles such that, for every $i=1, \ldots, m$, the triangle $U_{i}$ has a common edge with some $U_{k(i)}$ for $0 \leq k(i)<i$. Indeed, choose any triangle $U_{0}$ from the triangulation. Next choose any neighbouring triangle as $U_{1}$ and set $k(1)=0$. Now suppose that for some $1 \leq \ell \leq m$ we have chosen triangles $\left\{U_{i}: 0 \leq i \leq \ell\right\}$ such that $k(i)<i$ for $1 \leq i \leq \ell$. If $\ell=m$ then the process is over. If $\ell<m$ then choose as $U_{\ell+1}$ any triangle that has a common boundary with $\bigcup_{i=0}^{\ell} U_{i}$. This triangle exists by connectedness. Finally, let $0 \leq k(\ell+1) \leq l$ be such that $U_{\ell+1}$ has a common edge with $U_{k(\ell+1)}$.

For every $i=1, \ldots, m$ consider a small isosceles right triangle $W_{i} \subset U_{i} \cup U_{k(i)}$ such that its shortest height lies on the common edge of $U_{i}$ and $U_{k(i)}$ and $W_{i} \cap W_{j}=\emptyset$ whenever $i \neq j$. Furthermore, we assume that each of the triangles $W_{i}$ is of the same size and we choose a parametrization such that $W_{i}$ has vertices in points $(0,-a),(0, a)$ and $(a, 0)$, with $(0,-a) \in U_{i}$ for each $i=1, \ldots, m$ (see Fig. 2).

Let $B=\left[b_{i j}\right]_{i, j \in\{1, \ldots, m\}}$ be the matrix given by

$$
b_{i j}= \begin{cases}1 & \text { if } i=j \\ -1 & \text { if } i=k(j) \\ 0 & \text { otherwise }\end{cases}
$$

For each positive $f \in \mathcal{W}\left(M, \frac{1}{1+\varepsilon}, \frac{1}{1-\varepsilon}\right)$, denote by $v^{f} \in \mathbb{R}^{m}$ the solution of the following system of linear equations

$$
B v^{f}=\left[\lambda_{\zeta}\left(U_{i}\right)-\mu_{f}\left(U_{i}\right)\right]_{i=1, \ldots m} .
$$

Then $f \mapsto v^{f}$ is continuous. Let $\hat{\varepsilon}>0$ be such that Lemma 5.6 can be applied to any triangle $U_{i}$ (it exists due to Remark 5.7). Observe that if $f$ is constant equal to 1 , then $v^{f}=(0, \ldots, 0)$. By the continuity of $f \mapsto v^{f}$ we can choose $0<\varepsilon<\frac{\hat{\varepsilon}}{3}$ such that

$$
\left|v_{i}^{f}\right|<\frac{a^{2} \hat{\varepsilon}}{12}
$$




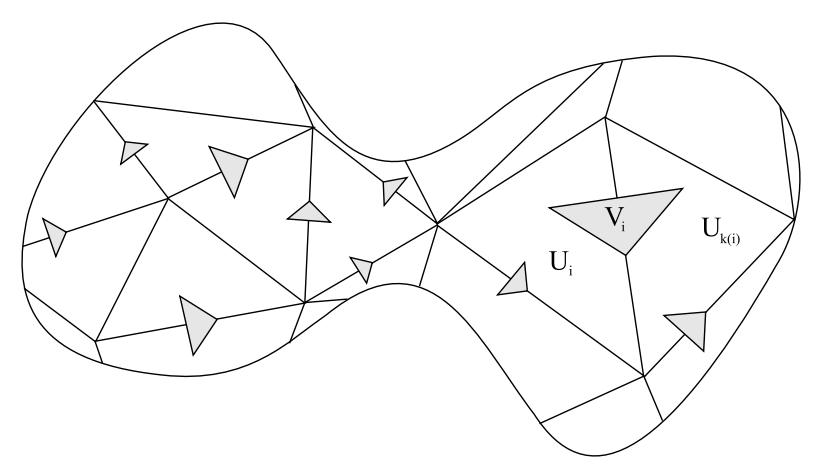

FiguRE 2. A triangulation of the surface $M$ and a choice of small triangles.

for every $f \in \mathcal{W}\left(M, \frac{1}{1+\varepsilon}, \frac{1}{1-\varepsilon}\right)$.

Let $f \in \mathcal{W}\left(M, \frac{1}{1+\varepsilon}, \frac{1}{1-\varepsilon}\right)$. We now construct a family of piecewise affine homeomorphisms $G_{f}^{i}: W_{i} \rightarrow W_{i}, i=1, \ldots, m$, such that $G_{f}^{i}$ depends continuously on $f,\left.G_{f}^{i}\right|_{\partial W_{i}}=I d$,

$$
1-\frac{\hat{\varepsilon}}{3}<\operatorname{det}\left(D\left(G_{f}^{i}\right)^{-1}(x)\right)<1+\frac{\hat{\varepsilon}}{3} \text { whenever } D\left(G_{f}^{i}\right)^{-1}(x) \text { is well defined, }
$$

and finally we require that the homeomorphism $\mathcal{G}_{f}: M \rightarrow M$ defined by

$$
\mathcal{G}_{f}(x):= \begin{cases}G_{f}^{i}(x) & \text { if } x \in W_{i} \text { for some } i=1, \ldots, m, \\ x & \text { otherwise }\end{cases}
$$

satisfies

$$
\left(\mathcal{G}_{f}\right)_{*} \mu_{f}\left(U_{j}\right)=\lambda_{\zeta}\left(U_{j}\right) \text { for all } 0 \leq j \leq m .
$$

First note that for each $i=1, \ldots, m$ we can choose $-1<h_{f}^{i}<1$ such that the quadrilateral $Q_{f}^{i} \subset W_{i}$ with vertices in points $(0,0)(0,-a),(a, 0)$ and $y_{f}^{i}:=\left(\sqrt{\hat{\varepsilon}} a\left(1-\left|h_{f}^{i}\right|\right), h_{f}^{i} a\right)$ has measure $\mu_{f}$ equal to $\mu_{f}\left(W_{i} \cap U_{i}\right)+v_{i}^{f}$. Indeed,

$$
\mu_{f}\left(W_{i} \cap U_{i}\right) \geq \frac{1}{1+\varepsilon} \lambda_{\zeta}\left(W_{i} \cap U_{i}\right)=\frac{a^{2}}{2(1+\varepsilon)} \geq \frac{a^{2}}{4}>\left|v_{i}^{f}\right|
$$

and analogously

$$
\mu_{f}\left(W_{i} \cap U_{k(i)}\right)>\left|v_{i}^{f}\right|
$$

Thus

$$
0<\mu_{f}\left(W_{i} \cap U_{i}\right)+v_{i}^{f}<\mu_{f}\left(W_{i}\right),
$$

which, together with the absolute continuity of $\mu_{f}$, yields the existence of $h_{f}^{i}$ for $i=1, \ldots, m$.

We now estimate $\left|h_{f}^{i}\right|$. Since $\left|v_{i}^{f}\right|$ is the $\mu_{f}$ measure of the triangle with vertices $(0,0),(a, 0)$ and $y_{f}^{i}$ in $W_{i}$ and $a\left|h_{f}^{i}\right|$ is its height, we have

$$
\left|v_{i}^{f}\right|>\frac{1}{1+\varepsilon} \frac{a^{2}\left|h_{f}^{i}\right|}{2} .
$$

Hence, by 68,

$$
\left|h_{f}^{i}\right|<\frac{2(1+\varepsilon)\left|v_{i}^{f}\right|}{a^{2}}<\frac{(1+\varepsilon) \hat{\varepsilon}}{6}<\frac{\hat{\varepsilon}}{3} .
$$

Let $G_{f}^{i}: W_{i} \rightarrow W_{i}$ be given by $G_{f}^{i}:=H\left(h_{f}^{i}, \sqrt{\hat{\varepsilon}}\right)$ for $i=1, \ldots, m$ as in 30 . Since

$$
\left(G_{f}^{i}\right)^{-1}\left(W_{i} \cap U_{i}\right)=Q_{f}^{i},
$$


we have

Analogously

$$
\left(\mathcal{G}_{f}\right)_{*} \mu_{f}\left(W_{i} \cap U_{i}\right)=\mu_{f}\left(Q_{f}^{i}\right)=\mu_{f}\left(W_{i} \cap U_{i}\right)+v_{i}
$$

$\left(\mathcal{G}_{f}\right)_{*} \mu_{f}\left(W_{i} \cap U_{k(i)}\right)=\mu_{f}\left(W_{i} \backslash Q_{f}^{i}\right)=\mu_{f}\left(W_{i}\right)-\left(\mu_{f}\left(W_{i} \cap U_{i}\right)+v_{i}\right)=\mu_{f}\left(W_{i} \cap U_{k(i)}\right)-v_{i}$.

By the definition of $v_{i}^{f}$ and by the fact that $W_{i}$ and $W_{j}$ are disjoint for $i \neq j$, we have for $i=1, \ldots, m$

$$
\begin{aligned}
& \left(\mathcal{G}_{f}\right)_{*}\left(\mu_{f}\right)\left(U_{i}\right)=\left(\mathcal{G}_{f}\right)_{*}\left(\mu_{f}\right)\left(U_{i} \backslash\left(W_{i} \cup \bigcup_{\{j ; k(j)=i\}} W_{j}\right)\right) \\
& \quad+\left(\mathcal{G}_{f}\right)_{*}\left(\mu_{f}\right)\left(W_{i} \cap U_{i}\right)+\sum_{\{j ; k(j)=i\}}\left(\mathcal{G}_{f}\right)_{*}\left(\mu_{f}\right)\left(W_{j} \cap U_{i}\right) \\
& \quad=\mu_{f}\left(U_{i} \backslash\left(W_{i} \cup \bigcup_{\{j ; k(j)=i\}} W_{j}\right)\right)+\left(\mu_{f}\left(W_{i} \cap U_{i}\right)+v_{i}^{f}\right)+\sum_{\{j ; k(j)=i\}}\left(\mu_{f}\left(W_{j} \cap U_{i}\right)-v_{j}^{f}\right) \\
& =\mu_{f}\left(U_{i}\right)+v_{i}^{f}-\sum_{\{j ; k(j)=i\}} v_{j}^{f}=\mu_{f}\left(U_{i}\right)+\left(B v^{f}\right)_{i}=\lambda_{\zeta}\left(U_{i}\right) .
\end{aligned}
$$

Since $\left(G_{f}^{i}\right)_{*}\left(\mu_{f}\right)\left(W_{i}\right)=\mu_{f}\left(W_{i}\right)$, we also have

$$
\left(\mathcal{G}_{f}\right)_{*}\left(\mu_{f}\right)(M)=\mu_{f}(M)=\lambda_{\zeta}(M),
$$

and thus

$$
\left(\mathcal{G}_{f}\right)_{*}\left(\mu_{f}\right)\left(U_{0}\right)=\lambda_{\zeta}\left(U_{0}\right)
$$

Hence 70 is satisfied. Moreover (71) together with $(32)$ and $(33)$ yield 69 . What is left to prove is that $G_{f}^{i}$ depends continuously on $f$. By Lemma 5.2 we only need to prove that $h_{f}^{i}$ depends continuously on $f$.

Take $f \in \mathcal{W}\left(M, \frac{1}{1+\varepsilon}, \frac{1}{1-\varepsilon}\right)$, and let $\delta>0$. Since $v^{g}$ depends continuously on $g$, there exists $0<\delta^{\prime} \leq \delta$ such that, for any $g \in \mathcal{W}\left(M, \frac{1}{1+\varepsilon}, \frac{1}{1-\varepsilon}\right)$,

$$
\|f-g\|_{L^{1}}<\delta^{\prime} \Longrightarrow \max _{i=1, \ldots, m}\left|v_{i}^{f}-v_{i}^{g}\right|<\delta
$$

We now evaluate the difference between the respective Lebesgue measures of the quadrilaterals given by $(0,0),(0,-a),(a, 0), y_{f}^{i}$ and by $(0,0),(0,-a),(a, 0), y_{g}^{i}$ :

$$
\begin{aligned}
\frac{a^{2}\left|h_{f}^{i}-h_{g}^{i}\right|}{2}< & (1+\varepsilon)\left|\mu_{f}\left(W_{i} \cap U_{i}\right)+v_{i}^{f}-\mu_{g}\left(W_{i} \cap U_{i}\right)-v_{i}^{g}+\int_{W_{i}}\right| f-g\left|d \lambda_{\zeta}\right| \\
& \leq(1+\varepsilon)\left(\left|\mu_{f}\left(W_{i} \cap U_{i}\right)-\mu_{g}\left(W_{i} \cap U_{i}\right)\right|+\left|v_{i}^{f}-v_{i}^{g}\right|+\int_{W_{i}}|f-g| d \lambda_{\zeta}\right) \\
& <(1+\varepsilon)\left(\int_{W_{i} \cap U_{i}}|f-g| d \lambda_{\zeta}+\delta+\int_{W_{i}}|f-g| d \lambda_{\zeta} \mid\right) \leq(1+\varepsilon)\left(2\|f-g\|_{L^{1}}+\delta\right) .
\end{aligned}
$$

Hence, for every $g \in \mathcal{W}\left(M, \frac{1}{1+\varepsilon}, \frac{1}{1-\varepsilon}\right)$ such that $\|f-g\|_{L^{1}}<\delta^{\prime}$, we have

$$
\left|h_{f}^{i}-h_{g}^{i}\right|<\frac{6(1+\varepsilon) \delta}{a^{2}},
$$

which implies that $h_{f}^{i}$ depends continuously on $f$.

Since $G_{f}^{i}$ depends continuously on $f$, by the definition $\mathcal{G}_{f}$ also depends continuously on $f$. Since $f \in \mathcal{W}\left(M, \frac{1}{1+\varepsilon}, \frac{1}{1-\varepsilon}\right)$, and $\varepsilon<\frac{\hat{\varepsilon}}{3}$, by $\left[69,\left(\mathcal{G}_{f}\right)_{*} \mu_{f}\right.$ is an absolutely continuous measure with density $\hat{f}=\left(f \circ \mathcal{G}_{f}^{-1}\right) \cdot \operatorname{det} D\left(\mathcal{G}_{f}^{-1}\right)$ satisfying

$$
\frac{1}{1+\hat{\varepsilon}}<\frac{1-\frac{\hat{\varepsilon}}{3}}{1+\frac{\hat{\varepsilon}}{3}}<\hat{f}<\frac{1+\frac{\hat{\varepsilon}}{3}}{1-\frac{\hat{\varepsilon}}{3}}<\frac{1}{1-\hat{\varepsilon}}
$$


and

$$
\int_{U_{i}} \hat{f} d \lambda_{\zeta}=\left(\mathcal{G}_{f}\right)_{*} \mu_{f}\left(U_{i}\right)=\lambda_{\zeta}\left(U_{i}\right) \text { for every } i=0, \ldots, m
$$

Therefore, on each $U_{i}$ the density $\hat{f}$ satisfies the assumptions of Lemma 5.6 . Hence, for each $i=0, \ldots, m$, there exists a homeomorphism $H_{\hat{f}}^{i}: U_{i} \rightarrow U_{i}$ which transports the measure $\left.\left(\mathcal{G}_{f}\right)_{*} \mu_{f}\right|_{U_{i}}$ to $\left.\lambda_{\zeta}\right|_{U_{i}}$, and such that $\left.H_{\hat{f}}^{i}\right|_{\partial U_{i}}=I d$. Therefore, we can define a homeomorphism $H_{\hat{f}}: M \rightarrow M$ such that

$$
H_{\hat{f}}(x):=H_{\hat{f}}^{i}(x) \text { whenever } x \in U_{i} .
$$

Then $\left(H_{\hat{f}} \circ \mathcal{G}_{f}\right)_{*} \mu_{f}=\left(H_{\hat{f}}\right)_{*}\left(\left(\mathcal{G}_{f}\right)_{*} \mu_{f}\right)=\lambda_{\zeta}$. Let $\mathcal{H}_{f}:=H_{\hat{f}} \circ \mathcal{G}_{f}$. What is left to prove is that $f \mapsto \mathcal{H}_{f}$ is continuous.

By Lemma $5.6 H_{\hat{f}}^{i}$ depend continuously on $\hat{f}$ and hence $\hat{f} \mapsto H_{\hat{f}}$ is continuous. Moreover, (v) in Lemma 5.3 implies that $f \mapsto \operatorname{det}\left(D \mathcal{G}_{f}^{-1}\right) \in L^{\infty}(M)$ is continuous. Furthermore, by (iv) in Lemma 5.3. the homeomorphism $\mathcal{G}_{f}^{-1}: M \rightarrow M$ is Lipschitz with constant $\frac{5}{4}$. Thus, by Lemma 5.4 and Remark 5.5, $f \mapsto f \circ \mathcal{G}_{f}^{-1}$ is continuous. Hence

$$
\mathcal{W}\left(M, \frac{1}{1+\varepsilon}, \frac{1}{1-\varepsilon}\right) \ni f \mapsto \hat{f}=\left(f \circ \mathcal{G}_{f}^{-1}\right) \cdot \operatorname{det} D\left(\mathcal{G}_{f}^{-1}\right) \in L^{1}(M)
$$

is continuous and this implies the continuity of $f \mapsto H_{\hat{f}}$.

Now consider any $f \in \mathcal{W}\left(M, \frac{1}{1+\varepsilon}, \frac{1}{1-\varepsilon}\right)$. Since $H_{\hat{f}}: M \rightarrow M$ is uniformly continuous, for any $\eta>0$ we can find $0<\delta$ such that

$$
d_{M}(x, y)<\delta \Rightarrow d_{M}\left(H_{\hat{f}}(x), H_{\hat{f}}(y)\right)<\eta .
$$

Then, for every $x \in M$ and any $g \in \mathcal{W}\left(M, \frac{1}{1+\varepsilon}, \frac{1}{1-\varepsilon}\right)$ such that $d_{\text {Hom }}\left(H_{\hat{f}}, H_{\hat{g}}\right)<\eta$ and $d_{\text {Hom }}\left(\mathcal{G}_{f}, \mathcal{G}_{g}\right)<\delta$, we have

$$
\begin{aligned}
d_{M}\left(H_{\hat{f}} \circ \mathcal{G}_{f}(x), H_{\hat{g}} \circ \mathcal{G}_{g}(x)\right) \leq & d_{M}\left(H_{\hat{f}} \circ \mathcal{G}_{f}(x), H_{\hat{f}} \circ \mathcal{G}_{g}(x)\right) \\
& +d_{M}\left(H_{\hat{f}} \circ \mathcal{G}_{g}(x), H_{\hat{g}} \circ \mathcal{G}_{g}(x)\right)<2 \eta .
\end{aligned}
$$

Analogously,

$$
d_{M}\left(\left(H_{\hat{f}} \circ \mathcal{G}_{f}\right)^{-1}(x),\left(H_{\hat{g}} \circ \mathcal{G}_{g}\right)^{-1}(x)\right)<2 \eta .
$$

This concludes the proof of the continuity of $f \mapsto \mathcal{H}_{f}$ and the proof of the whole theorem.

\section{Local CONTINUOUS EMBEDding OF THE MOdULi SPACE}

In this section, we finalize the construction of a continuous mappings on open subsets of a connected component of any stratum, which is needed to prove the main result of this paper. We do it in two steps.

Firstly, for each $\zeta \in \mathcal{M}(M, \Sigma, \kappa)$ we construct a neighborhood $\mathcal{U}_{\zeta} \subset \mathcal{M}(M, \Sigma, \kappa)$ of $\zeta$, so that for every $\omega \in \mathcal{U}_{\zeta}$ there exists a piecewise affine homeomorphism $\mathfrak{h}_{\omega}: M \rightarrow M$ such that $\left(\mathfrak{h}_{\omega}\right)_{*} \lambda_{\omega}=f_{\omega} \lambda_{\zeta}$ with $\frac{1}{1+\varepsilon_{\zeta}}<f_{\omega}<\frac{1}{1-\varepsilon_{\zeta}}$, where $\varepsilon_{\zeta}>0$ is given by Theorem 5.8 . We will also require that $\omega \mapsto f_{\omega} \in L^{1}(M)$ is continuous.

Secondly, we use the results of the previous sections to show the existence of a homeomorphism $\mathcal{H}_{\omega}: M \rightarrow M$ such that $\left(\mathcal{H}_{\omega} \circ \mathfrak{h}_{\omega}\right)_{*} \lambda_{\omega}=\lambda_{\zeta}$. Moreover, we show that these homeomorphisms yield the existence of a continuous mapping $\mathfrak{S}: \mathcal{U}_{\zeta} \rightarrow \operatorname{Flow}(M, \zeta)$ such that $\mathfrak{S}(\omega)$ is isomorphic by a homeomorphism to $\mathcal{T}^{\omega}$ - the vertical translation flow on $(M, \omega)$.

Lemma 6.1. Let $\zeta \in \mathcal{M}(M, \Sigma, \kappa)$. There exists a neigbourhood $\mathcal{U}_{\zeta} \subset M(M, \Sigma, \kappa)$ such that, for every $\omega \in \mathcal{U}_{\zeta}$, the following holds:

(i) there exists a triangulation $\mathcal{Y}(\omega)$ and a piecewise affine homeomorphism $\mathfrak{h}_{\omega}:(M, \omega) \rightarrow$ $(M, \zeta)$ which is affine on elements of $\mathcal{Y}(\omega)$, fixes $\Sigma$ and is Lipschitz with constant $\frac{11}{10}$,

(ii) $\left(\mathfrak{h}_{\omega}\right)_{*} \lambda_{\omega}$ is an absolutely continuous measure with respect to $\lambda_{\zeta}$ with piecewise constant density $f_{\omega}$ satisfying $\frac{1}{1+\varepsilon_{\zeta}}<f_{\omega}<\frac{1}{1-\varepsilon_{\zeta}}$, 
(iii) the mapping $U_{\zeta} \ni \omega \mapsto f_{\omega} \in L^{1}\left(M, \lambda_{\zeta}\right)$ is continuous.

Moreover, for given $\epsilon>0$, there exists $\delta>0$ such that

(iv) for any $\bar{\omega} \in \mathcal{U}_{\zeta}$, if $d_{M o d}(\omega, \bar{\omega})<\delta$ then $\mathfrak{h}_{\bar{\omega}}^{-1} \circ \mathfrak{h}_{\bar{\omega}}:(M, \bar{\omega}) \rightarrow(M, \omega)$ is affine on elements of $\mathcal{Y}(\bar{\omega}), \mathfrak{h}_{\bar{\omega}}^{-1} \circ \mathfrak{h}_{\omega}:(M, \omega) \rightarrow(M, \bar{\omega})$ is affine on elements of $\mathcal{Y}(\omega)$, they are both Lipschitz with constant $1+\epsilon$,

$$
\left\|I d-\left.D\left(\mathfrak{h}_{\omega}^{-1} \circ \mathfrak{h}_{\bar{\omega}}\right)\right|_{A}\right\|<\epsilon \text { for every } A \in \mathcal{Y}(\bar{\omega})
$$

and

$$
\left\|I d-\left.D\left(\mathfrak{h}_{\bar{\omega}}^{-1} \circ \mathfrak{h}_{\omega}\right)\right|_{B}\right\|<\epsilon \text { for every } B \in \mathcal{Y}(\omega),
$$

(v) for any $\bar{\omega} \in \mathcal{U}_{\zeta}$ such that $d_{M o d}(\omega, \bar{\omega})<\delta$ and for the set

$$
\tilde{M}(\omega):=\left\{x \in M ; \inf _{\sigma \in \Sigma} d_{\omega}\left(\mathcal{T}_{t}^{\omega}(x), \sigma\right)>4 \epsilon \text { for all } t \in[-1,1]\right\},
$$

we have $\lambda_{\omega}(\tilde{M}(\omega))>1-K \epsilon$, where $K>0$ depends only on stratum, and for $x \in \tilde{M}(\omega)$ we have

$$
d_{\omega}\left(\mathcal{T}_{t}^{\omega}(x), \mathfrak{h}_{\omega}^{-1} \circ \mathfrak{h}_{\bar{\omega}} \circ \mathcal{T}_{t}^{\bar{\omega}} \circ \mathfrak{h}_{\bar{\omega}}^{-1} \circ \mathfrak{h}_{\omega}(x)\right)<\epsilon \text { for any } t \in[-1,1]
$$

and for every $\sigma \in \Sigma$

$$
d_{\omega}\left(\sigma, \mathfrak{h}_{\omega}^{-1} \circ \mathfrak{h}_{\bar{\omega}} \circ \mathcal{T}_{t}^{\bar{\omega}} \circ \mathfrak{h}_{\bar{\omega}}^{-1} \circ \mathfrak{h}_{\omega}(x)\right)>3 \epsilon \text { for any } t \in[-1,1]
$$

Proof. Let $\pi$ be a permutation of the alphabet $\mathcal{A}$ with $d$ elements which belongs to a Rauzy class corresponding to $\mathcal{M}(M, \Sigma, \kappa)$. We can assume that $\zeta$ has no vertical saddle-connections and thus there is a polygonal representation $\left(\pi, \lambda^{\zeta}, \tau^{\zeta}\right)$ of $\zeta$. Otherwise we can rotate $\zeta$ to obtain a form $\zeta^{\prime}$ which does not admit vertical saddle connections, construct a triangulation $\mathcal{Y}\left(\zeta^{\prime}\right)$ and rotate it back together with this triangulation to obtain a triangulation $\mathcal{Y}(\zeta)$ (note that a rotation is an isometry and that it acts continuously on $\mathcal{M}(M, \Sigma, \kappa)$, see [23]).

Let $\omega \in \mathcal{M}(M, \Sigma, \kappa)$, and assume that $\omega=M\left(\pi, \lambda^{\omega}, \tau^{\omega}\right)$ for some $\lambda^{\omega}, \tau^{\omega}$. Let $\mathcal{P}(\omega) \subset \mathbb{C}$ be the polygon corresponding to $\omega$, whose vertices $R_{0}(\omega), R_{1}(\omega), \ldots, R_{d}(\omega), R_{1}^{\prime}(\omega), \ldots, R_{d-1}^{\prime}(\omega)$ are given by

$$
R_{i}(\omega):=\sum_{\left\{\alpha ; \pi_{0}(\alpha) \leq i\right\}}\left(\lambda_{\alpha}^{\omega}+i \tau_{\alpha}^{\omega}\right) \quad \text { and } \quad R_{i}^{\prime}(\omega):=\sum_{\left\{\alpha ; \pi_{1}(\alpha) \leq i\right\}}\left(\lambda_{\alpha}^{\omega}+i \tau_{\alpha}^{\omega}\right) \quad \text { for } i=0, \ldots, d .
$$

Note that $R_{0}(\omega)=R_{0}^{\prime}(\omega)=0, R_{d}(\omega)=R_{d}^{\prime}(\omega)$. For $i=1, \ldots, d-1$ consider the vertical segments connecting $R_{i}(\omega)$ and $R_{i}^{\prime}(\omega)$ with the opposite side of $\mathcal{P}(\omega)$. Denote the other endpoints of those segments by $Q_{i}(\omega)$ and $Q_{i}^{\prime}(\omega)$ respectively (see Fig. 3). Since each side on the upper half of the polygon is identified with one of the sides on the lower half of the polygon, there exist representations of $Q_{i}(\omega)$ and $Q_{i}^{\prime}(\omega)$ on the opposite half of the polygon which we denote by $S_{i}(\omega)$ and $S_{i}^{\prime}(\omega)$ respectively. Note that

$$
\operatorname{Re}\left(S_{i}(\omega)\right)=T_{\pi, \lambda^{\omega}}^{-1}\left(\operatorname{Re}\left(R_{i}(\omega)\right)\right) \text { and } \operatorname{Re}\left(S_{i}^{\prime}(\omega)\right)=T_{\pi, \lambda^{\omega}}\left(\operatorname{Re}\left(R_{i}^{\prime}(\omega)\right)\right),
$$

where $T_{\pi, \lambda \omega}$ is the IET given by $(\pi, \lambda)$. Let

$$
\mathcal{V}(\omega):=\left\{R_{0}(\omega), \ldots, R_{d}(\omega), R_{1}^{\prime}(\omega), \ldots, R_{d-1}^{\prime}(\omega), S_{1}(\omega), \ldots, S_{d-1}(\omega), S_{1}^{\prime}(\omega), \ldots, S_{d-1}^{\prime}(\omega)\right\} .
$$

$\mathcal{V}(\omega)$ is fully determined by $\left(\lambda^{\omega}, \tau^{\omega}\right)$. If $\omega$ has no vertical saddle connections, then for all distinct $x, y \in \mathcal{V}(\omega)$ we have $\operatorname{Re}(x) \neq \operatorname{Re}(y)$. Consider the sequence $\left\{V_{j}(\omega)\right\}_{j=0}^{4 d-2}$, which is an ordering of $\mathcal{V}(\omega)$, such that $\left\{\operatorname{Re}\left(V_{j}\right)\right\}_{j=0}^{4 d-2}$ be an increasing sequence.

Let $\varepsilon_{1}>0$ be such that for every $\omega \in \mathcal{M}(M, \Sigma, \kappa)$ satisfying $d_{M o d}(\zeta, \omega)<\varepsilon_{1}$, the orderings of the sets $\mathcal{V}(\zeta)$ and $\mathcal{V}(\omega)$ are the same, that is

$$
\begin{gathered}
V_{j}(\omega)=R_{i}(\omega) \text { iff } V_{j}(\zeta)=R_{i}(\zeta) ; \quad V_{j}(\omega)=R_{i}^{\prime}(\omega) \text { iff } V_{j}(\zeta)=R_{i}^{\prime}(\zeta), \\
V_{j}(\omega)=S_{i}(\omega) \text { iff } V_{j}(\zeta)=S_{i}(\zeta) \quad \text { and } \quad V_{j}(\omega)=S_{i}^{\prime}(\omega) \text { iff } V_{j}(\zeta)=S_{i}^{\prime}(\zeta),
\end{gathered}
$$

for every $j=0, \ldots, 2 d$, and $\left\{\operatorname{Re}\left(V_{i}(\omega)\right)\right\}_{i=0}^{d}$ is strictly increasing.

For every $\omega \in \mathcal{M}(M, \Sigma, \kappa)$ with $d_{M o d}(\zeta, \omega)<\varepsilon_{1}$, we now construct a triangulation $\mathcal{Y}(\omega)$ of $\mathcal{P}(\omega)$. (We abuse the word "triangulation" since the edges may connect vertices of triangulation 


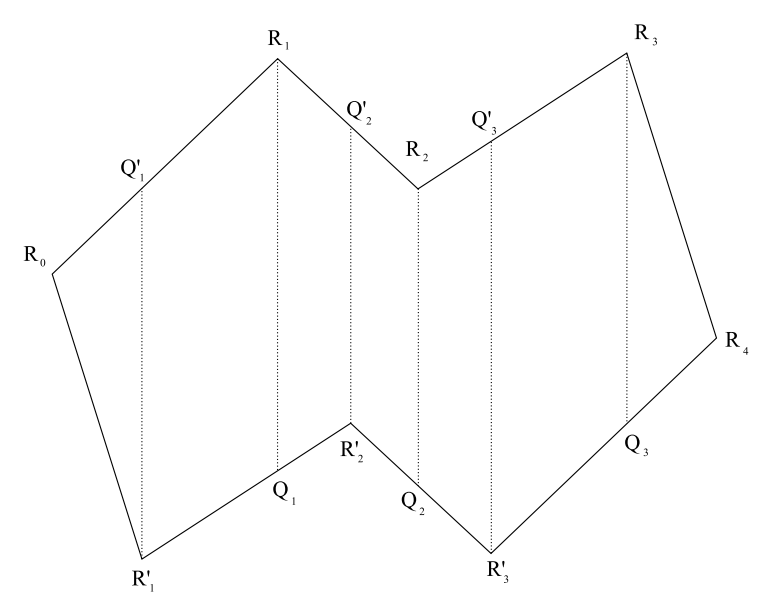

FigURE 3. The vertices of $\mathcal{P}(\omega)$ and their projections on opposite sides.

which are actually the same points.) Let $\{r(k)\}_{0 \leq k \leq 2 d}$ be the strictly increasing sequence in $\{0, \ldots, 4 d-2\}$ such that $V_{r(0)}, \ldots, V_{r(2 d)}$ be the vertices of $\mathcal{P}(\omega)$. Let $\tilde{V}_{r(k)}(\omega):=Q_{i}(\omega)$ whenever $V_{r(k)}(\omega)=R_{i}(\omega)$ and analogously let $\tilde{V}_{r(k)}(\omega):=Q_{i}^{\prime}(\omega)$ whenever $V_{r(k)}(\omega)=R_{i}^{\prime}(\omega)$.

Consider the triangle given by the points $V_{r(0)}=0, V_{r(1)}, \tilde{V}_{r(1)}$. If $r(1)=1$, then this triangle belongs to $\mathcal{Y}(\omega)$. If $r(1) \neq 1$ and $\operatorname{Im}\left(V_{1}(\omega)\right)>0$, then we connect by segments all $V_{i}(\omega)$ such that $i \leq r(1)$ and $\operatorname{Im}\left(V_{i}(\omega)\right)<0$ with $V_{1}(\omega)$,and for all $i \leq r(1)$ such that $\operatorname{Im}\left(V_{i}(\omega)\right)>0$, we connect $V_{i}(\omega)$ with the point $V_{r(1)}(\omega)$ or $\tilde{V}_{r(1)}(\omega)$, whichever has the negative imaginary part. If $r(1) \neq 1$ and $\operatorname{Im}\left(V_{1}(\omega)\right)<0$, then we proceed symmetrically. The triangles obtained by using the above segments are elements of $\mathcal{Y}(\omega)$. By applying vertical reflection, we use the same construction for the triangle given by points $V_{r(2 d-1)}(\omega), \tilde{V}_{r(2 d-1)}(\omega), V_{r(2 d)}(\omega)$.

For every $k=1, \ldots, 2 d-2$, consider the trapezoid given by $V_{r(k)}(\omega), V_{r(k+1)}(\omega), \tilde{V}_{r(k)}(\omega)$ and $\tilde{V}_{r(k+1)}(\omega)$. In each of those trapezoids, we take the diagonal connecting the top-left vertex with the bottom-right vertex. If $r(k+1)=r(k)+1$, then the two resulting triangles belong to $\mathcal{Y}(\omega)$. If $r(k+1) \neq r(k)+1$, then for every $r(k)<i<r(k+1)$, we connect $V_{i}(\omega)$ with the bottom-right vertex if $\operatorname{Im}\left(V_{i}(\omega)\right)>0$, and with the top-left vertex if $\operatorname{Im}\left(V_{i}(\omega)\right)<0$. We include the resulting triangles into $\mathcal{Y}(\omega)$. In this way we get a triangulation $\mathcal{Y}(\omega)$ of $\mathcal{P}(\omega)$ into triangles which have vertices in $\mathcal{V}(\omega)$ (see Fig. 4).

Define $\mathfrak{h}_{\omega}:(M, \omega) \rightarrow(M, \zeta)$ as the piecewise affine transformation, such that

$$
\mathfrak{h}_{\omega}\left(V_{i}(\omega)\right)=V_{i}(\zeta) \text { for every } i=0, \ldots, 4 d-2
$$

and sending affinely each triangle from $\mathcal{Y}(\omega)$ with vertices $V_{j}(\omega), V_{k}(\omega), V_{\ell}(\omega)$ onto the triangle with vertices $V_{j}(\zeta), V_{k}(\zeta), V_{\ell}(\zeta)$. Note that the map $\mathfrak{h}_{\omega}$ is uniquely determined by the points in $\mathcal{V}(\omega)$. Moreover, since $\Sigma \subset \mathcal{V}(\omega), \mathfrak{h}_{\omega}$ fixes $\Sigma$.

Let $0<\varepsilon_{2}<\varepsilon_{1}$ be such that, defining $\tilde{\mathcal{U}}_{\zeta}:=\left\{\omega \in \mathcal{M}(M, \Sigma, \kappa), d_{M o d}(\zeta, \omega)<\varepsilon_{2}\right\}$, we have

$$
\omega \in \tilde{\mathcal{U}}_{\zeta} \Rightarrow 1-\varepsilon_{\zeta}<\frac{\lambda_{\zeta}(A)}{\lambda_{\omega}\left(\mathfrak{h}_{\omega}^{-1}(A)\right)}<1+\varepsilon_{\zeta} \text { for every } A \in \mathcal{Y}(\zeta) .
$$

This implies that $\left(\mathfrak{h}_{\omega}\right)_{*} \lambda_{\omega}$ is absolutely continuous with respect to $\lambda_{\zeta}$ and has a piecewise constant density $f_{\omega}$ given by

$$
f_{\omega}(x)=\frac{\lambda_{\omega}\left(\mathfrak{h}_{\omega}^{-1}(A)\right)}{\lambda_{\zeta}(A)} \text { for every } x \in A \text { where } A \in \mathcal{Y}(\zeta) .
$$

Hence

$$
\frac{1}{1+\varepsilon_{\zeta}}<f_{\omega}<\frac{1}{1-\varepsilon_{\zeta}}
$$




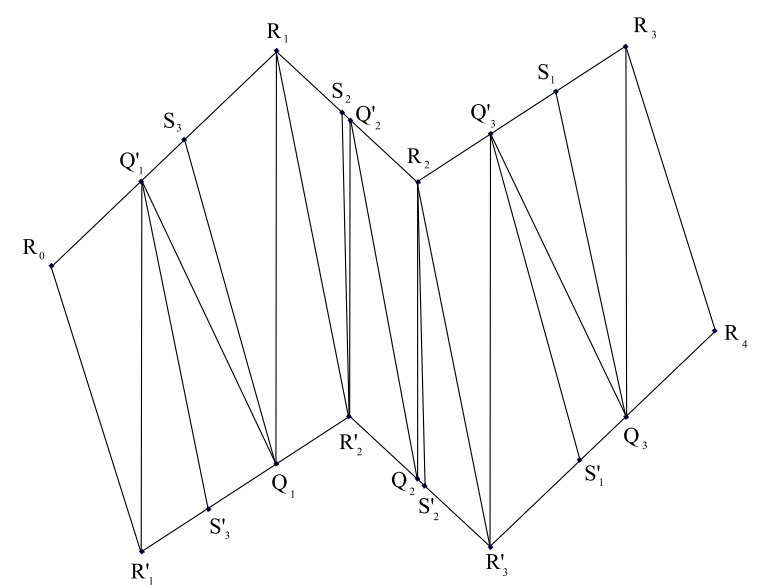

Figure 4. The triangulation $\mathcal{Y}(\omega)$ for the polygon in Fig. 3.

Moreover, note that for every $A \in \mathcal{Y}(\zeta)$, the mapping $\omega \rightarrow \lambda_{\omega}\left(\mathfrak{h}_{\omega}^{-1}(A)\right)$ is continuous. Hence, the formula 73 implies the continuity of $\omega \mapsto f_{\omega} \in L^{1}\left(M, \lambda_{\zeta}\right)$.

Let $\omega \in \tilde{\mathcal{U}}_{\zeta}$. Then for any $\bar{\omega} \in \tilde{\mathcal{U}}_{\zeta}, \mathfrak{h}_{\bar{\omega}}^{-1} \circ \mathfrak{h}_{\omega}$ is a continuous piecewise affine homoeomorphism which is affine on the elements of $\mathcal{Y}(\omega)$ and

$$
\mathfrak{h}_{\bar{\omega}}^{-1} \circ \mathfrak{h}_{\omega}\left(V_{i}(\omega)\right)=V_{i}(\bar{\omega})
$$

Take any $A \in \mathcal{Y}(\zeta)$ and let $V_{j}(\zeta), V_{k}(\zeta), V_{\ell}(\zeta) \in \mathcal{V}(\zeta)$ be its vertices. Then $\operatorname{lin}\left(\left.\mathfrak{h}_{\bar{\omega}}^{-1} \circ \mathfrak{h}_{\omega}\right|_{\mathfrak{h}_{\omega}^{-1} A}\right)$ is given by a matrix $B_{A}(\omega, \bar{\omega})=\left[b_{i j}(\omega, \bar{\omega})\right]_{i, j=1,2}$, where

$$
\begin{aligned}
b_{1,1}(\omega, \bar{\omega}) & =\frac{\operatorname{Re}\left(V_{k}(\bar{\omega})-V_{j}(\bar{\omega})\right) \operatorname{Im}\left(V_{\ell}(\omega)-V_{j}(\omega)\right)-\operatorname{Re}\left(V_{\ell}(\bar{\omega})-V_{j}(\bar{\omega})\right) \operatorname{Im}\left(V_{k}(\omega)-V_{j}(\omega)\right)}{\operatorname{Re}\left(V_{k}(\omega)-V_{j}(\omega)\right) \operatorname{Im}\left(V_{\ell}(\omega)-V_{j}(\omega)\right)-\operatorname{Re}\left(V_{\ell}(\omega)-V_{j}(\omega)\right) \operatorname{Im}\left(V_{k}(\omega)-V_{j}(\omega)\right)}, \\
b_{1,2}(\omega, \bar{\omega}) & =\frac{\operatorname{Re}\left(V_{\ell}(\bar{\omega})-V_{j}(\bar{\omega})\right) \operatorname{Re}\left(V_{k}(\omega)-V_{j}(\omega)\right)-\operatorname{Re}\left(V_{k}(\bar{\omega})-V_{j}(\bar{\omega})\right) \operatorname{Re}\left(V_{\ell}(\omega)-V_{j}(\omega)\right)}{\operatorname{Re}\left(V_{k}(\omega)-V_{j}(\omega)\right) \operatorname{Im}\left(V_{\ell}(\omega)-V_{j}(\omega)\right)-\operatorname{Re}\left(V_{\ell}(\omega)-V_{j}(\omega)\right) \operatorname{Im}\left(V_{k}(\omega)-V_{j}(\omega)\right)}, \\
b_{2,1}(\omega, \bar{\omega}) & =\frac{\operatorname{Im}\left(V_{k}(\bar{\omega})-V_{j}(\bar{\omega})\right) \operatorname{Im}\left(V_{\ell}(\omega)-V_{j}(\omega)\right)-\operatorname{Im}\left(V_{\ell}(\bar{\omega})-V_{j}(\bar{\omega})\right) \operatorname{Im}\left(V_{k}(\omega)-V_{j}(\omega)\right)}{\operatorname{Re}\left(V_{k}(\omega)-V_{j}(\omega)\right) \operatorname{Im}\left(V_{\ell}(\omega)-V_{j}(\omega)\right)-\operatorname{Re}\left(V_{\ell}(\omega)-V_{j}(\omega)\right) \operatorname{Im}\left(V_{k}(\omega)-V_{j}(\omega)\right)},
\end{aligned}
$$

and

$$
b_{2,2}(\omega, \bar{\omega})=\frac{\operatorname{Re}\left(V_{k}(\omega)-V_{j}(\omega)\right) \operatorname{Im}\left(V_{\ell}(\bar{\omega})-V_{j}(\bar{\omega})\right)-\operatorname{Im} \operatorname{Re}\left(V_{\ell}(\omega)-V_{j}(\omega)\right)\left(V_{k}(\bar{\omega})-V_{j}(\bar{\omega})\right)}{\operatorname{Re}\left(V_{k}(\omega)-V_{j}(\omega)\right) \operatorname{Im}\left(V_{\ell}(\omega)-V_{j}(\omega)\right)-\operatorname{Re}\left(V_{\ell}(\omega)-V_{j}(\omega)\right) \operatorname{Im}\left(V_{k}(\omega)-V_{j}(\omega)\right)} .
$$

Note that, to obtain a formula for $B_{A}(\bar{\omega}, \omega)=\operatorname{lin}\left(\left.\mathfrak{h}_{\omega}^{-1} \circ \mathfrak{h}_{\bar{\omega}}\right|_{\mathfrak{h}_{\bar{\omega}}^{-1} A}\right)$, it is enough to switch $\omega$ with $\bar{\omega}$. Observe that $B_{A}(\omega, \omega)=I d$. Since all coefficients depend continuously on the elements of $\mathcal{V}(\bar{\omega})$ and $\mathfrak{h}_{\zeta}=I d$, by taking $\bar{\omega}=\zeta$ we can find $0<\varepsilon_{3} \leq \varepsilon_{2}$ such that for all $\omega \in \mathcal{U}_{\zeta}:=\{\omega \in$ $\left.\mathcal{M}(M, \Sigma, \kappa), d_{M o d}(\zeta, \omega)<\varepsilon_{3}\right\}, \mathfrak{h}_{\omega}$ and $\mathfrak{h}_{\omega}^{-1}$ are Lipschitz with constant $\frac{11}{10}$. Moreover, for any $\epsilon>0$ and any $\omega \in \mathcal{U}_{\zeta}$ we can find $\delta>0$ such that for all $A \in \mathcal{Y}(\zeta)$ and for all $\bar{\omega}$ satisfying $d_{M o d}(\omega, \bar{\omega})<\delta$, we have that $\mathfrak{h}_{\omega}^{-1} \circ \mathfrak{h}_{\bar{\omega}}$ and $\mathfrak{h}_{\bar{\omega}}^{-1} \circ \mathfrak{h}_{\omega}$ are Lipschitz with constant $1+\epsilon$ and $\left\|I d-B_{A}(\omega, \bar{\omega})\right\|<\epsilon$ and $\left\|I d-B_{A}(\bar{\omega}, \omega)\right\|<\epsilon$.

To prove (v), note that the set $\tilde{M}(\omega)$ does not contain points which are in the $4 \epsilon$-neighbourhood of ingoing and outgoing vertical separatrix segments of length 1 starting from singular points. That is the complement $\tilde{M}(\omega)^{c}$ is of measure $\lambda_{\omega}$ at most $(1+4 \epsilon) 8 \epsilon$ times the number of ingoing and outgoing separatrices, which determines the value of $K$.

For every $\bar{\omega} \in \mathcal{U}_{\zeta}$ denote by $X^{\bar{\omega}}: M \rightarrow \mathbb{R}^{2}$ the unit constant vertical vector field on $(M, \bar{\omega})$ defined on $M \backslash \Sigma$ which generates $\mathcal{T}^{\bar{\omega}}$. Then by (iv) we have that

$$
\left\|X^{\omega}(x)-D\left(\mathfrak{h}_{\omega}^{-1} \circ \mathfrak{h}_{\bar{\omega}}\right)_{h_{\bar{\omega}}^{-1} \circ \mathfrak{h}_{\omega}(x)} X^{\bar{\omega}}\left(\mathfrak{h}_{\bar{\omega}}^{-1} \circ \mathfrak{h}_{\omega}(x)\right)\right\|<\epsilon .
$$


Note that the vector field $D\left(\mathfrak{h}_{\omega}^{-1} \circ \mathfrak{h}_{\bar{\omega}}\right)_{h_{\bar{\omega}}^{-1} \circ \mathfrak{h}_{\omega}(x)} X^{\bar{\omega}}\left(h_{\bar{\omega}}^{-1} \circ \mathfrak{h}_{\omega}(x)\right)$ is well defined everywhere except on the edges of the triangulation $\mathcal{Y}(\omega)$. Since this vector field is constant on the interiors of the elements of the triangulation $\mathcal{Y}(\omega)$, we can define it on $M \backslash \Sigma$ by choosing on each edge of $\mathcal{Y}(\omega)$ one of the vectors derived from one of the two triangles forming this edge. It is worth to mention that, if the direction of an edge of $\mathcal{Y}(\bar{\omega})$ given by some triangles $\mathfrak{h}_{\bar{\omega}}^{-1}(A)$ and $\mathfrak{h}_{\bar{\omega}}^{-1}(B) \in \mathcal{Y}(\bar{\omega})$ coincides with the direction of the flow $\mathcal{T}^{\bar{\omega}}$, then the vector field $D\left(\mathfrak{h}_{\omega}^{-1} \circ \mathfrak{h}_{\bar{\omega}}\right)_{h_{\bar{\omega}}^{-1} \circ \mathfrak{h}_{\omega}(x)} X^{\bar{\omega}}\left(h_{\bar{\omega}}^{-1} \circ\right.$ $\left.\mathfrak{h}_{\omega}(x)\right)$ takes the same values on the triangles $\mathfrak{h}_{\omega}^{-1}(A), \mathfrak{h}_{\omega}^{-1}(B) \in \mathcal{Y}(\omega)$. Hence the flow induced by this vector field is well defined and (74) holds everywhere on $M \backslash \Sigma$. Thus, in view of (74), for any $t \in[-1,1]$ we have

$$
\begin{aligned}
& \left|\int_{0}^{t}\left\|X^{\omega}\left(\mathcal{T}_{s}^{\omega}(x)\right)-D\left(\mathfrak{h}_{\omega}^{-1} \circ \mathfrak{h}_{\bar{\omega}}\right)_{\mathcal{T}_{s}^{\bar{\omega}} \circ \mathfrak{h}_{\bar{\omega}}^{-1} \circ \mathfrak{h}_{\omega}(x)} X^{\bar{\omega}}\left(\mathcal{T}_{s}^{\bar{\omega}} \circ \mathfrak{h}_{\bar{\omega}}^{-1} \circ \mathfrak{h}_{\omega}(x)\right)\right\| d s\right| \\
= & \left|\int_{0}^{t}\left\|X^{\omega}\left(\mathfrak{h}_{\omega}^{-1} \circ \mathfrak{h}_{\bar{\omega}} \circ \mathcal{T}_{s}^{\bar{\omega}} \circ \mathfrak{h}_{\bar{\omega}}^{-1} \circ \mathfrak{h}_{\omega}(x)\right)-D\left(\mathfrak{h}_{\omega}^{-1} \circ \mathfrak{h}_{\bar{\omega}}\right)_{\mathcal{T}_{s}^{\bar{\omega}} \circ \mathfrak{h}_{\bar{\omega}}^{-1} \circ \mathfrak{h}_{\omega}(x)} X^{\bar{\omega}}\left(\mathcal{T}_{s}^{\bar{\omega}} \circ \mathfrak{h}_{\bar{\omega}}^{-1} \circ \mathfrak{h}_{\omega}(x)\right)\right\| d s\right| \\
< & |t| \epsilon \leq \epsilon .
\end{aligned}
$$

Since for every $x \in \tilde{M}(\omega)$ we have

$$
\mathfrak{h}_{\omega}^{-1} \circ \mathfrak{h}_{\bar{\omega}} \circ \mathcal{T}_{t}^{\bar{\omega}} \circ \mathfrak{h}_{\bar{\omega}}^{-1} \circ \mathfrak{h}_{\omega}(x)=x+\int_{0}^{t} D\left(\mathfrak{h}_{\omega}^{-1} \circ \mathfrak{h}_{\bar{\omega}}\right)_{\mathcal{T}_{s}^{\bar{\omega}} \circ \mathfrak{h}_{\bar{\omega}}^{-1} \circ \mathfrak{h}_{\omega}(x)} X^{\bar{\omega}}\left(\mathcal{T}_{s}^{\bar{\omega}}\left(\mathfrak{h}_{\bar{\omega}}^{-1} \circ \mathfrak{h}_{\omega}(x)\right)\right) d s
$$

in local coordinates, we deduce that

$$
\begin{aligned}
& d_{\omega}\left(\mathcal{T}_{t}^{\omega}(x), \mathfrak{h}_{\omega}^{-1} \circ \mathfrak{h}_{\bar{\omega}} \circ \mathcal{T}_{t}^{\bar{\omega}} \circ \mathfrak{h}_{\bar{\omega}}^{-1} \circ \mathfrak{h}_{\omega}(x)\right) \\
& \quad \leq\left|\int_{0}^{t}\left\|X^{\omega}\left(\mathcal{T}_{s}^{\omega}(x)\right)-D\left(\mathfrak{h}_{\omega}^{-1} \circ \mathfrak{h}_{\bar{\omega}}\right)_{\mathcal{T}_{s}^{\bar{\omega}} \circ \mathfrak{h}_{\bar{\omega}}^{-1} \circ \mathfrak{h}_{\omega}(x)} X^{\bar{\omega}}\left(\mathcal{T}_{s}^{\bar{\omega}}\left(\mathfrak{h}_{\bar{\omega}}^{-1} \circ \mathfrak{h}_{\omega}(x)\right)\right)\right\| d s\right| \leq \epsilon
\end{aligned}
$$

Since $x \in \tilde{M}(\omega)$, this also implies that for every $\sigma \in \Sigma$ we have

$$
d_{\omega}\left(\sigma, \mathfrak{h}_{\omega}^{-1} \circ \mathfrak{h}_{\bar{\omega}} \circ \mathcal{T}_{t}^{\bar{\omega}} \circ \mathfrak{h}_{\bar{\omega}}^{-1} \circ \mathfrak{h}_{\omega}(x)\right)>3 \epsilon
$$

This concludes the proof of $(\mathrm{v})$ and thus the proof of the whole lemma.

Lemma 6.2. Let $\omega \in \mathcal{M}(M, \Sigma, \kappa)$ and let $D$ be a rectangle in $(M, \omega)$. For every $\varepsilon>0$ there exists $\delta>0$ such that, for every $\lambda_{\omega}$-measure preserving $F: D \rightarrow(M, \omega)$ satisfying

$$
\sup _{x \in D} d_{\omega}(x, F(x))<\delta
$$

we have

$$
\lambda_{\omega}(D)-\lambda_{\omega}(D \cap F(D))<\varepsilon .
$$

Proof. Let $\varepsilon>0$. We assume that $\varepsilon<\lambda_{\omega}(D)$, otherwise the result is obvious. Choose $\delta>0$ such that the set

$$
\hat{D}:=\left\{x \in D ; \forall y \in M, d_{\omega}(x, y)<\delta \Rightarrow y \in D\right\}
$$

has measure $\lambda_{\omega}(\hat{D})>\lambda_{\omega}(D)-\varepsilon$. Then, for any $\lambda_{\omega}$-measure preserving $F: D \rightarrow(M, \omega)$ satisfying 75, , we have $F(\hat{D}) \subset D$. Hence

$$
\lambda_{\omega}(D \cap F(D)) \geq \lambda_{\omega}(F(\hat{D}))=\lambda_{\omega}(\hat{D})>\lambda_{\omega}(D)-\varepsilon .
$$

Theorem 6.3. Let $\zeta \in \mathcal{M}(M, \Sigma, \kappa)$ and let $\mathcal{U}_{\zeta}$ be the neighbourhood given by Lemma 6.1. There exists a continuous mapping $\mathfrak{S}: \mathcal{U}_{\zeta} \rightarrow \operatorname{Flow}\left(M, \lambda_{\zeta}\right)$ such that for every $\omega \in \mathcal{U}_{\zeta}$ the vertical flow on $(M, \omega)$ is measure-theoretically isomorphic by a homeomorphism to the measure-preserving flow $\mathfrak{S}(\omega)$ on $\left(M, \lambda_{\zeta}\right)$. 
Proof. By (i) and (ii) in Lemma 6.1 for every $\omega \in \mathcal{U}_{\zeta}$ there exists a homeomorphism $\mathfrak{h}_{\omega}: M \rightarrow$ $M$ fixing $\Sigma$ and such that $\left(\mathfrak{h}_{\omega}\right)_{*} \lambda_{\omega}=f_{\omega} \lambda_{\zeta}$, where $f_{\omega}$ satisfies $\frac{1}{1+\varepsilon_{\zeta}}<f_{\omega}<\frac{1}{1-\varepsilon_{\zeta}}$. Hence we can apply Theorem 5.8 to obtain a homeomorphism $\mathcal{H}_{\omega}:=\mathcal{H}_{f_{\omega}}: M \rightarrow M$, which depends continuously on $f_{\omega}$ and $\left(\mathcal{H}_{f_{\omega}}\right)_{*}\left(f_{\omega} \lambda_{\zeta}\right)=\lambda_{\zeta}$. By (iii) in Lemma 6.1 it follows that the map $\omega \mapsto f_{\omega}$ is continuous. Hence $\omega \mapsto \mathcal{H}_{\omega}$, as a composition of two continuous mappings, is also continuous. Now define a homeomorphism of $M$

$$
\mathcal{S}_{\omega}:=\mathcal{H}_{\omega} \circ \mathfrak{h}_{\omega} .
$$

Note that $\left(\mathcal{S}_{\omega}\right)_{*} \lambda_{\omega}=\lambda_{\zeta}$ and the flow $\mathcal{S}_{\omega} \circ \mathcal{T}^{\omega} \circ \mathcal{S}_{\omega}^{-1}$ is $\lambda_{\zeta}$-measure preserving. To conclude the proof we show now that the mapping $\mathcal{U}_{\zeta} \ni \omega \mapsto \mathcal{S}_{\omega} \circ \mathcal{T}^{\omega} \circ \mathcal{S}_{\omega}^{-1}=: \mathfrak{S}(\omega) \in \operatorname{Flow}\left(M, \lambda_{\zeta}\right)$ is continuous.

Fix $\omega \in \mathcal{U}_{\zeta}$. We now prove the continuity of $\mathfrak{S}$ in $\omega$. On $(M, \omega)$ choose a family $\mathcal{Q}$ of open rectangles, with vertical and horizontal sides, that generates the Borel $\sigma$-algebra on $M$. We may assume that for every $Q \in \mathcal{Q}$ we have $\lambda_{\omega}(Q) \leq \frac{1}{4}$. Note that $\mathcal{S}_{\omega}^{-1}:(M, \zeta) \rightarrow(M, \omega)$ is a measure-theoretic isomorphism. Hence, in view of Remark 2.6 to prove that $\mathfrak{S}$ is continuous, it is sufficient to prove that the $\operatorname{map} \mathcal{U}_{\zeta} \ni \bar{\omega} \mapsto \mathcal{S}_{\omega}^{-1} \circ \mathfrak{S}(\bar{\omega})_{t} \circ \mathcal{S}_{\omega} \in$ Flow $\left(X, \lambda_{\omega}\right)$ is continuous. That is for every $\varepsilon>0$ and $Q \in \mathcal{Q}$ there exists $\delta>0$ such that

$$
d_{\text {Mod }}(\omega, \bar{\omega})<\delta \Rightarrow \sup _{t \in[-1,1]} \lambda_{\omega}\left(\mathcal{T}_{t}^{\omega} Q \triangle \mathcal{S}_{\omega}^{-1} \circ \mathfrak{S}(\bar{\omega})_{t} \circ \mathcal{S}_{\omega} Q\right)<\varepsilon .
$$

Fix $Q \in \mathcal{Q}$ and $\varepsilon>0$. We now prove (77) for $Q$. Denote by $k$ the number of times the ingoing and outgoing vertical separatrix segments of length 2 starting from the singular points $\sigma \in \Sigma$ intersect with $Q$. By extending those segments if necessary, we obtain segments $v_{j} \subset Q$, for $j=1, \ldots, k$, such that the endpoints of $v_{j}$ lie on a horizontal sides of $Q$. Let $0<\epsilon_{Q}<\varepsilon$, and consider the subset $\tilde{Q} \subseteq Q$ obtained by cutting out from $Q$ all rectangles of which the segments $v_{j}$ are vertical sides and whose width is $4 \epsilon_{Q}$. Assume that $\epsilon_{Q}$ is small enough so that

$$
\lambda_{\omega}(\tilde{Q})>(1-\varepsilon) \lambda_{\omega}(Q) .
$$

Note that $\tilde{Q}$ is a union of $l \leq k+1$ rectangles $D_{j}$ for $j=1, \ldots, l$. By Lemma 6.2 there exists $\gamma>0$ such that for every $j=1, \ldots, l$ and every $\lambda_{\omega}$-preserving transformation $F: D_{j} \rightarrow M$ satisfying $\sup _{x \in D_{j}} d_{\omega}(x, F(x))<4 \gamma$ we have

$$
\lambda_{\omega}\left(D_{j} \cap F\left(D_{j}\right)\right)>(1-\varepsilon) \lambda_{\omega}\left(D_{j}\right) .
$$

Take $0<\epsilon<\min \left\{\gamma, \epsilon_{Q}\right\}$. Since $\bar{\omega} \mapsto \mathcal{H}_{\bar{\omega}}$ is continuous, we can choose $\delta>0$ such that for every $\bar{\omega} \in U_{\zeta}$

$$
d_{\text {Mod }}(\omega, \bar{\omega})<\delta \Longrightarrow\left(\sup _{x \in M} d_{\zeta}\left(\mathcal{H}_{\omega}^{-1} \circ \mathcal{H}_{\bar{\omega}}(x), x\right)<\epsilon \quad \wedge \sup _{x \in M} d_{\zeta}\left(\mathcal{H}_{\bar{\omega}}^{-1} \circ \mathcal{H}_{\omega}(x), x\right)<\epsilon\right) .
$$

Moreover, by applying (iv) from Lemma 6.1 for $\epsilon$ and taking smaller $\delta$ if necessary, we get that $\mathfrak{h}_{\omega}^{-1} \circ \mathfrak{h}_{\bar{\omega}}:(M, \bar{\omega}) \rightarrow(M, \omega)$ and $\mathfrak{h}_{\bar{\omega}}^{-1} \circ \mathfrak{h}_{\omega}:(M, \omega) \rightarrow(M, \bar{\omega})$ are Lipschitz piecewise affine homeomorphisms with constant $1+\epsilon$. Furthermore, (v) in Lemma 6.1 gives us that the set $\tilde{M}(\omega)$ satisfies $\lambda_{\omega}(\tilde{M}(\omega))>1-K \epsilon$ and for $x \in \tilde{M}(\omega)$ we have

$$
d_{\omega}\left(\mathcal{T}_{t}^{\omega}(x), \mathfrak{h}_{\omega}^{-1} \circ \mathfrak{h}_{\bar{\omega}} \circ \mathcal{T}_{t}^{\bar{\omega}} \circ \mathfrak{h}_{\bar{\omega}}^{-1} \circ \mathfrak{h}_{\omega}(x)\right)<\epsilon \text { for any } t \in[-1,1]
$$

It also implies that, for every $\sigma \in \Sigma$,

$$
d_{\omega}\left(\sigma, \mathfrak{h}_{\omega}^{-1} \circ \mathfrak{h}_{\bar{\omega}} \circ \mathcal{T}_{t}^{\bar{\omega}} \circ \mathfrak{h}_{\bar{\omega}}^{-1} \circ \mathfrak{h}_{\omega}(x)\right)>3 \epsilon \text { for any } t \in[-1,1] .
$$

Since $\epsilon<\epsilon_{Q}$, we have $\tilde{Q} \subset \tilde{M}(\omega)$.

We now estimate the distance between the orbits of the flows $\mathfrak{h}_{\omega}^{-1} \circ \mathfrak{h}_{\bar{\omega}} \circ \mathcal{T}_{t}^{\bar{\omega}} \circ \mathfrak{h}_{\bar{\omega}}^{-1} \circ \mathfrak{h}_{\omega}$ and

$$
\mathcal{S}_{\omega}^{-1} \circ \mathcal{S}_{\bar{\omega}} \circ \mathcal{T}_{t}^{\bar{\omega}} \circ \mathcal{S}_{\bar{\omega}}^{-1} \circ \mathcal{S}_{\omega}=\mathfrak{h}_{\omega}^{-1} \circ \mathcal{H}_{\omega}^{-1} \circ \mathcal{H}_{\bar{\omega}} \circ \mathfrak{h}_{\bar{\omega}} \circ T_{t}^{\bar{\omega}} \circ h_{\bar{\omega}}^{-1} \circ \mathcal{H}_{\bar{\omega}}^{-1} \circ \mathcal{H}_{\omega} \circ h_{\omega}
$$

By (80) we have that

$$
d_{\zeta}\left(\mathfrak{h}_{\omega}(x), \mathcal{H}_{\bar{\omega}}^{-1} \circ \mathcal{H}_{\omega} \circ \mathfrak{h}_{\omega}(x)\right)<\epsilon
$$


for every $x \in M$. By (i) in Lemma 6.1 $\mathfrak{h}_{\bar{\omega}}^{-1}:(M, \zeta) \rightarrow(M, \omega)$ is Lipschitz with constant $\frac{11}{10}$. Thus we have

$$
d_{\bar{\omega}}\left(\mathfrak{h}_{\bar{\omega}}^{-1} \circ \mathfrak{h}_{\omega}(x), \mathfrak{h}_{\bar{\omega}}^{-1} \circ \mathcal{H}_{\bar{\omega}}^{-1} \circ \mathcal{H}_{\omega} \circ \mathfrak{h}_{\omega}(x)\right)<\frac{11}{10} \epsilon .
$$

Since $\mathfrak{h}_{\omega}^{-1} \circ \mathfrak{h}_{\bar{\omega}}$ is Lipschitz with constant $1+\epsilon$ and fixes $\Sigma$, 82 implies that

$$
\min _{\sigma \in \Sigma} \inf _{t \in[-1,1]} d_{\bar{\omega}}\left(\mathcal{T}_{t}^{\bar{\omega}} \circ \mathfrak{h}_{\bar{\omega}}^{-1} \circ \mathfrak{h}_{\omega}(x), \sigma\right)>\frac{3 \epsilon}{1+\epsilon}>2 \epsilon
$$

for every $x \in \tilde{M}(\omega)$. Hence on the $2 \epsilon$-neighbourhood of $\mathfrak{h}_{\bar{\omega}}^{-1} \circ \mathfrak{h}_{\omega}(x),\left\{\mathcal{T}_{t}^{\bar{\omega}}\right\}_{t \in[-1,1]}$ acts isometrically. Thus

$$
d_{\bar{\omega}}\left(\mathcal{T}_{t}^{\bar{\omega}}\left(\mathfrak{h}_{\bar{\omega}}^{-1} \circ \mathfrak{h}_{\omega}(x)\right), \mathcal{T}_{t}^{\bar{\omega}} \circ \mathfrak{h}_{\bar{\omega}}^{-1} \circ \mathcal{H}_{\bar{\omega}}^{-1} \circ \mathcal{H}_{\omega} \circ \mathfrak{h}_{\omega}(x)\right)<\frac{11}{10} \epsilon,
$$

for $t \in[-1,1]$ and for every $x \in \tilde{M}(\omega)$. Since $\mathfrak{h}_{\bar{\omega}}:(M, \bar{\omega}) \rightarrow(M, \zeta)$ is Lipschitz with constant $\frac{11}{10}$, this implies that

$$
d_{\zeta}\left(\mathfrak{h}_{\bar{\omega}} \circ \mathcal{T}_{t}^{\bar{\omega}} \circ \mathfrak{h}_{\bar{\omega}}^{-1} \circ \mathfrak{h}_{\omega}(x), \mathfrak{h}_{\bar{\omega}} \circ \mathcal{T}_{t}^{\bar{\omega}} \circ \mathfrak{h}_{\bar{\omega}}^{-1} \circ \mathcal{H}_{\bar{\omega}}^{-1} \circ \mathcal{H}_{\omega} \circ \mathfrak{h}_{\omega}(x)\right)<\frac{121}{100} \epsilon .
$$

Again by using 80 we obtain that

$$
d_{\zeta}\left(\mathfrak{h}_{\bar{\omega}} \circ \mathcal{T}_{t}^{\bar{\omega}} \circ \mathfrak{h}_{\bar{\omega}}^{-1} \circ \mathfrak{h}_{\omega}(x), \mathcal{H}_{\omega}^{-1} \circ \mathcal{H}_{\bar{\omega}} \circ \mathfrak{h}_{\bar{\omega}} \circ \mathcal{T}_{t}^{\bar{\omega}} \circ \mathfrak{h}_{\bar{\omega}}^{-1} \circ \mathcal{H}_{\bar{\omega}}^{-1} \circ \mathcal{H}_{\omega} \circ \mathfrak{h}_{\omega}(x)\right)<\frac{221}{100} \epsilon .
$$

Finally, since $\mathfrak{h}_{\omega}^{-1}$ is also Lipschitz with constant $\frac{11}{10}$, we obtain that

$d_{\omega}\left(\mathfrak{h}_{\omega}^{-1} \circ \mathfrak{h}_{\bar{\omega}} \circ \mathcal{T}_{t}^{\bar{\omega}} \circ h_{\bar{\omega}}^{-1} \circ h_{\omega}(x), \mathfrak{h}_{\omega}^{-1} \circ \mathcal{H}_{\omega}^{-1} \circ \mathcal{H}_{\bar{\omega}} \circ \mathfrak{h}_{\bar{\omega}} \circ \mathcal{T}_{t}^{\bar{\omega}} \circ h_{\bar{\omega}}^{-1} \circ \mathcal{H}_{\bar{\omega}}^{-1} \circ \mathcal{H}_{\omega} \circ h_{\omega}(x)\right)<\frac{2431}{1000} \epsilon$.

By combining this with 81 we obtain that for every $x \in \tilde{M}(\omega)$ we have

$$
d_{\omega}\left(\mathcal{T}_{t}^{\omega}(x), \mathcal{S}_{\omega}^{-1} \circ \mathfrak{S}(\bar{\omega})_{t} \circ \mathcal{S}_{\omega}(x)\right)<\frac{3431}{1000} \epsilon<4 \epsilon .
$$

By the definition of $\tilde{M}(\omega),\left\{\mathcal{T}_{t}^{\omega}\right\}_{t \in[-1,1]}$ acts isometrically on the $4 \epsilon$-neighbourhood of $x \in \tilde{M}(\omega)$. Hence

$$
d_{\omega}\left(x, \mathcal{T}_{-t}^{\omega} \circ \mathcal{S}_{\omega}^{-1} \circ \mathfrak{S}(\bar{\omega})_{t} \circ \mathcal{S}_{\omega}(x)\right)<4 \epsilon .
$$

Since $D_{j} \subseteq \tilde{Q} \subset \tilde{M}(\omega)$, 84 is satisfied for all $x \in D_{j}$. Consider

$$
F:=\mathcal{T}_{-t}^{\omega} \circ \mathcal{S}_{\omega}^{-1} \circ \mathfrak{S}(\bar{\omega})_{t} \circ \mathcal{S}_{\omega} .
$$

Note that $F$ is $\lambda_{\omega}$-measure preserving. Thus, by $(79)$, we get

$$
\lambda_{\omega}\left(D_{j} \cap \mathcal{T}_{-t}^{\omega} \circ \mathcal{S}_{\omega}^{-1} \circ \mathfrak{S}(\bar{\omega})_{t} \circ \mathcal{S}_{\omega}\left(D_{j}\right)\right)>(1-\varepsilon) \lambda_{\omega}\left(D_{j}\right) .
$$

Together with $\mathcal{T}^{\omega}$-invariance of $\lambda_{\omega}$, this yields

$$
\lambda_{\omega}\left(\mathcal{T}_{t}^{\omega}\left(D_{j}\right) \cap \mathcal{S}_{\omega}^{-1} \circ \mathfrak{S}(\bar{\omega})_{t} \circ \mathcal{S}_{\omega}\left(D_{j}\right)\right)>(1-\varepsilon) \lambda_{\omega}\left(\mathcal{T}_{t}^{\omega}\left(D_{j}\right)\right),
$$

for every $t \in[-1,1]$. By summing up over $j=1, \ldots, l$ we get

$$
\lambda_{\omega}\left(\mathcal{T}_{t}^{\omega}(\tilde{Q}) \cap \mathcal{S}_{\omega}^{-1} \circ \mathfrak{S}(\bar{\omega})_{t} \circ \mathcal{S}_{\omega}(\tilde{Q})\right)>(1-\varepsilon) \lambda_{\omega}\left(\mathcal{T}_{t}^{\omega}(\tilde{Q})\right)
$$

and by $(78)$ this yields

$$
\lambda_{\omega}\left(\mathcal{T}_{t}^{\omega}(Q) \cap \mathcal{S}_{\omega}^{-1} \circ \mathfrak{S}(\bar{\omega})_{t} \circ \mathcal{S}_{\omega}(Q)\right)>(1-2 \varepsilon) \lambda_{\omega}\left(\mathcal{T}_{t}^{\omega}(Q)\right) .
$$

Since $\lambda_{\omega}(Q)<\frac{1}{4}$, we have

$$
\lambda_{\omega}\left(\mathcal{T}_{t}^{\omega}(Q) \triangle \mathcal{S}_{\omega}^{-1} \circ \mathfrak{S}(\bar{\omega})_{t} \circ \mathcal{S}_{\omega}(Q)\right)<4 \varepsilon \lambda_{\omega}\left(\mathcal{T}_{t}^{\omega}(Q)\right) \leq \varepsilon .
$$

Thus we get (77), and this concludes the proof of the theorem.

Since the construction above is local, we need to show that this suffices to transport the $G_{\delta}$-condition from the space of flows to the moduli space. 
Lemma 6.4. Let $X$ be a metric topological space. Let $\left\{U_{i}\right\}_{i \in \mathbb{N}}$ be a sequence of open subsets such that $\bigcup_{i \in \mathbb{N}} U_{i}=X$. If $V \subseteq X$ is such that

$V \cap U_{i}$ is a $G_{\delta}$-set for each $i \in \mathbb{N}$,

then $V$ is a $G_{\delta}$-set.

Proof. Note that $V=\bigcap_{i \in \mathbb{N}}\left(V \cap U_{i}\right) \cup U_{i}^{c}$. Since $X$ is metrizable, every closed set is a $G_{\delta}$-set. To finish the proof it is enough to observe that the union of two $G_{\delta}$-sets is a $G_{\delta}$-set.

\section{Translation flows Disjoint With their inverses are $G_{\delta}$-DENSE}

We have the following result which follows from the proof of Corollary 3.3 in [5].

Theorem 7.1. Let $(X, \mathcal{B}(X), \mu)$ be a nonatomic standard Borel probability space, and let Flow $(X)$ be the space of $\mu$-invariant flows on $X$. The set of flows which are weakly mixing and disjoint with their inverse is $G_{\delta}$-dense in $\operatorname{Flow}(X)$.

The following result allows us to transfer the $G_{\delta}$ condition onto any connected component of the moduli space.

Proposition 7.2. Let $\mathfrak{P r o p}$ be a property of a measure-preserving flow such that the set of elements having this property is a $G_{\delta}$ subset of the space $\operatorname{Flow}(X)$. Then in every connected component $C$ of $\mathcal{M}$, the set of translation structures for which the vertical flow has the property $\mathfrak{P r o p}$ is a $G_{\delta}$ set in $C$.

Proof. Let $C$ be a connected component of $\mathcal{M}$. In view of Theorem 6.3 for every $\zeta \in C$ there exists an open neighbourhood $\mathcal{U}_{\zeta}$ of $\zeta$ and a continuous mapping $\mathfrak{S}_{\zeta}: \mathcal{U}_{\zeta} \rightarrow$ Flow $\left(M, \lambda_{\zeta}\right)$ such that for every $\omega \in \mathcal{U}_{\zeta}$ the vertical flow $\mathcal{T}^{\omega}$ is measure-theoretically isomorphic to $\mathfrak{S}_{\zeta}(\omega)$. Since $C$ is a topological manifold, it is $\sigma$-compact. Thus there exists a sequence $\left\{\zeta_{n}\right\}_{n \in \mathbb{N}}$ of translation structures such that $\bigcup_{n \in \mathbb{N}} \mathcal{U}_{\zeta_{n}}=C$. For each $n \in \mathbb{N}$ we have that

$$
\begin{aligned}
\mathcal{Y}_{\zeta_{n}}: & =\left\{\omega \in \mathcal{U}_{\zeta_{n}} ; \mathcal{T}^{\omega} \text { satisfies } \mathfrak{P r o p}\right\} \\
& =\left\{\omega \in \mathcal{U}_{\zeta_{n}} ; \mathfrak{S}_{\zeta_{n}}(\omega) \text { satisfies } \mathfrak{P r o p}\right\} \\
& =\mathfrak{S}_{\zeta_{n}}^{-1}\left\{\mathcal{T} \in \text { Flow }\left(M, \lambda_{\zeta_{n}}\right) ; \mathcal{T} \text { satisfies } \mathfrak{P r o p}\right\}
\end{aligned}
$$

is a $G_{\delta}$ set in $\mathcal{U}_{\zeta_{n}}$. By Lemma 6.4, this gives that the set of $\omega \in C$ such that $\mathcal{T}^{\omega}$ satisfies $\mathfrak{P r o p}$ is a $G_{\delta}$ set in $C$.

By combining Theorem 7.1 and Proposition 7.2 we get the following result.

Corollary 7.3. The set of translation structures $\zeta$ such that the vertical flow on $(M, \zeta)$ is weakly mixing and disjoint with its inverse is a $G_{\delta}$ set in every connected component $C$ of the moduli space.

Throughout this section we use the following notation. Let $C \subset \mathcal{M}$ be a non-hyperelliptic connected component of the moduli space, i.e. $C$ is not of the form $\mathcal{M}^{\text {hyp }}(2 g-2)$ or $\mathcal{M}^{\text {hyp }}(g-$ $1, g-1)$ for any $g \geq 2$. Let $\pi=\left(\pi_{0}, \pi_{1}\right)$ be a permutation of the alphabet $\mathcal{A}$ of $d$ elements from the corresponding extended Rauzy class satisfying

$$
\pi_{0}^{-1}(1)=\pi_{1}^{-1}(d) \text { and } \pi_{0}^{-1}(d)=\pi_{1}^{-1}(1) .
$$

This permutation exists due to Theorem 2.7 and by the choice of $C$, it is not symmetric. Let $\Omega_{\pi}$ be the translation matrix corresponding to $\pi$. By Corollary 4.2 there exist symbols $a_{1}, a_{2} \in \mathcal{A}$ such that $\left(\Omega_{\pi}\right)_{a_{1} a_{2}}=\left(\Omega_{\pi}\right)_{a_{2} a_{1}}=0$ and for any rationally independent vector $\tau \in \mathbb{R}^{\mathcal{A}}$ the numbers

$$
\left(\Omega_{\pi} \tau\right)_{a_{2}}-\left(\Omega_{\pi} \tau\right)_{a_{1}} \text { and }\left(\Omega_{\pi} \tau\right)_{a_{1}}-\left(\left(\Omega_{\pi} \tau\right)_{\pi_{0}^{-1}(1)}+\left(\Omega_{\pi} \tau\right)_{\pi_{0}^{-1}(d)}\right)
$$

are rationally independent. The proof of the following lemma goes along the same lines as the proof of Lemma 14 in [8]. It is mainly based on the recurrence of polygonal Rauzy-Veech induction. 
Lemma 7.4. The set

$$
C_{*}:=\left\{M(\pi, \lambda, \tau) \in C ;(\pi, \lambda, \tau) \in \Theta_{\pi}, \lambda_{a}=0 \text { for } a \in \mathcal{A} \backslash\left\{\pi_{0}^{-1}(1), \pi_{0}^{-1}(d), a_{1}, a_{2}\right\}\right\}
$$

is dense in $C$.

Before heading to the proof of the main result we give the proof of Proposition 1.2 which treats the density of translation structures on which the vertical flow is reversible.

Proof of Proposition 1.2. By following again the proof of Lemma 14 in [8] we prove that the set

$$
C_{* *}:=\left\{M(\pi, \lambda, \tau) \in C ;(\pi, \lambda, \tau) \in \Theta_{\pi}, \lambda_{a}=0 \text { for } a \in \mathcal{A} \backslash\left\{\pi_{0}^{-1}(1), \pi_{0}^{-1}(d)\right\}\right\}
$$

is dense in $C$. The vertical flow on $M(\pi, \lambda, \tau) \in C_{* *}$ is measure-theoretically isomorphic to the vertical flow on a torus given by $\left(\lambda_{\pi_{0}^{-1}(1)}, \lambda_{\pi_{0}^{-1}(1)}\right)$ and $\pi$ - the non-trivial permutation of two elements. Since the translation flows on tori are reversible, this concludes the proof.

The special representations of the vertical flows associated to the translation structures from $C_{*}$ as given in Lemma 7.4 are special flows over IETs of 3 intervals and under a roof functions which are piecewise constant and have discontinuity points which coincide with the discontinuity points of the IET and one additional discontinuity point inside the middle interval. After one step of either left- or right-hand side Rauzy-Veech induction we get a special representation over rotation and under a piecewise constant roof function with 4 discontinuity points. We now prove some properties of such flows.

Let $\alpha \in[0,1)$ be an irrational number, and let $T_{\alpha}: \mathbb{R} / \mathbb{Z} \rightarrow \mathbb{R} / \mathbb{Z}$ be the rotation by $\alpha$. For any $\beta \in[0,1)$ let

$$
\|\beta\|:=\min \{\{\beta\},\{1-\beta\}\} .
$$

Let $\left\{q_{n}\right\}_{n \in \mathbb{N}}$ be the sequence of partial denominators associated to $\alpha$. Recall that for every odd $n \in \mathbb{N}$, we have a pair of Rokhlin towers

$$
\left\{T_{\alpha}^{i}\left[-\left\|q_{n} \alpha\right\|, 0\right)\right\}_{i=0, \ldots, q_{n-1}-1} \quad \text { and } \quad\left\{T_{\alpha}^{i}\left[0,\left\|q_{n-1} \alpha\right\|\right)\right\}_{i=0, \ldots, q_{n}-1}
$$

for $n \geq 1$, which covers $\mathbb{R} / \mathbb{Z}$. As a corollary from Theorem 3.9 in [4] we get the following result (recall the definitions of $f^{(r)}$ and $\mathrm{Leb}^{f}$ from subsection 2.2, as well as the definition of $\mu_{t, s}$ given in (1)).

Proposition 7.5. Let $\left\{\left(T_{\alpha}^{f}\right)_{t}\right\}_{t \in \mathbb{R}}$ be the special flow over the rotation by $\alpha \in[0,1)$ under a positive roof function $f \in L^{2}\left([0,1)\right.$, Leb). Suppose that there exists a rigidity sequence $\left\{r_{n}\right\}_{n \in \mathbb{N}}$ for $T_{\alpha}$ (which is a subsequence of $\left\{q_{n}\right\}_{n \in \mathbb{N}}$ ) such that, setting $b_{n}:=r_{n} \int_{0}^{1} f(y) d y$, the sequence

$$
\left\{\int_{0}^{1}\left|f^{\left(r_{n}\right)}(x)-b_{n}\right|^{2} d x\right\}, n \in \mathbb{N}
$$

is bounded. Then there exists a probability measure $P \in \mathcal{P}\left(\mathbb{R}^{2}\right)$ such that, up to taking a subsequence,

$$
\left(f^{\left(2 r_{n}\right)}-2 b_{n}, f^{\left(r_{n}\right)}-b_{n}\right)_{*} \text { Leb } \rightarrow P \text { weakly }
$$

Moreover, along the same subsequence, we have

$$
\operatorname{Leb}_{2 b_{n}, b_{n}}^{f} \rightarrow \int_{\mathbb{R}^{2}} \operatorname{Leb}_{-t,-u}^{f} d P(t, u)
$$

To prove the next result we need the following remark.

Remark 7.6. Let $f:[0,1) \rightarrow \mathbb{R}$ be a piecewise constant function. Let $\beta_{1}, \ldots, \beta_{k}$ be the jumps of $f$ and let $d_{1}, \ldots, d_{k}$ be their respective values. Then for every $x \in[0,1)$ and every odd $n \in \mathbb{N}$,

$$
\sum_{i=0}^{q_{n}-1}\left(f\left(T_{\alpha}^{q_{n}+i}(x)\right)-f\left(T_{\alpha}^{i}(x)\right)\right)=\sum_{i=0}^{q_{n}-1} \sum_{j=1}^{k}-d_{j} \chi_{T_{\alpha}^{-i}\left[\beta_{j}, \beta_{j}+\left\|q_{n} \alpha\right\|\right)}(x) .
$$


Indeed, the expression $f\left(T_{\alpha}^{q_{n}+i}(x)\right)-f\left(T_{\alpha}^{i}(x)\right)$ takes non-zero value if and only if there is a discontinuity point $\beta_{j}$ in the interval $\left(T_{\alpha}^{q_{n}+i}(x), T_{\alpha}^{i}(x)\right]$. However, we have $T_{\alpha}^{q_{n}+i}(x)=T_{\alpha}^{i}(x)-$ $\left\|q_{n} \alpha\right\|$. Hence $\beta_{j} \in\left(T_{\alpha}^{q_{n}+i}(x), T_{\alpha}^{i}(x)\right]$ if and only if $x \in T_{\alpha}^{-i}\left[\beta_{j}, \beta_{j}+\left\|q_{n} \alpha\right\|\right)$. In other words, if we consider the (not necessarily disjoint) towers $U_{j}:=\bigcup_{i=0}^{q_{n}-1} T^{-i}\left[\beta_{j}, \beta_{j}+\left\|q_{n} \alpha\right\|\right)$ for $j=1, \ldots, k$, then

$$
\sum_{i=0}^{q_{n}-1}\left(f\left(T_{\alpha}^{q_{n}+i}(x)\right)-f\left(T_{\alpha}^{i}(x)\right)\right)=\sum_{j=1}^{k}-d_{j} \chi_{U_{j}}(x) .
$$

In particular, if $x \notin U_{j}$ for all $j=1, \ldots, k$, then $\sum_{i=0}^{q_{n}-1}\left(f\left(T_{\alpha}^{q_{n}+i}(x)\right)-f\left(T_{\alpha}^{i}(x)\right)\right)=0$. Otherwise, if $x \in U_{j}$ for some $j=1, \ldots, k$, then $d_{j}$ contributes to the value of the considered expression.

We need the following theorem which is a version of Theorem 7.3 in [4]. Recall that $\xi: \mathbb{R}^{2} \rightarrow$ $\mathbb{R}$ is defined by $\xi(t, u):=t-2 u$.

Lemma 7.7. There exists a set $\Lambda \subset \mathbb{R} / \mathbb{Z}$ of full Lebesgue measure such that, for every $\alpha \in \Lambda$, there exists a set $D_{\alpha} \in(\mathbb{R} / \mathbb{Z}) \times(\mathbb{R} / \mathbb{Z})$ of full Lebesgue measure with the property that, if $\left(\beta_{1}, \beta_{2}\right) \in D_{\alpha}$, then

- the numbers $0,1-\alpha, \beta_{1}$ and $\beta_{2}$ are distinct;

- for every piecewise constant positive function $f: \mathbb{R} / \mathbb{Z} \rightarrow \mathbb{R}$ with discontinuity points $0,1-\alpha, \beta_{1}, \beta_{2}$ and jumps $d_{\beta_{1}}$ and $d_{\beta_{2}}$ at $\beta_{1}$ and $\beta_{2}$ respectively, for the special flow $\left\{\left(T_{\alpha}^{f}\right)_{t}\right\}_{t \in \mathbb{R}}$ there exist probability measures $P, Q \in \mathcal{P}\left(\mathbb{R}^{2}\right)$ such that, up to a subsequence, we have

$\operatorname{Leb}_{2 b_{n}, b_{n}}^{f} \rightarrow \int_{\mathbb{R}^{2}} \operatorname{Leb}_{-t,-u}^{f} d P(t, u)$ and $\operatorname{Leb}_{-2 b_{n},-b_{n}}^{f} \rightarrow \int_{\mathbb{R}^{2}} \operatorname{Leb}_{-t,-u}^{f} d Q(t, u)$ weakly,

where $b_{n}:=q_{n} \int_{0}^{1} f(x) d x$. Moreover, $\xi_{*} P$ is atomic with exactly 4 atoms in points 0 , $-d_{\beta_{1}},-d_{\beta_{2}}, d_{\beta_{1}}+d_{\beta_{2}}$, while $\xi_{*} Q=(-\xi)_{*} P$ has exactly 4 atoms in points $0, d_{\beta_{1}}, d_{\beta_{2}}$, $-\left(d_{\beta_{1}}+d_{\beta_{2}}\right)$.

Proof. Let $\Lambda \subset \mathbb{R} / \mathbb{Z}$ be the set of irrational $\alpha \in \mathbb{R} / \mathbb{Z}$ such that there exists a sequence $\left\{k_{n}\right\}_{n \in \mathbb{N}}$ of odd numbers such that, for some $\frac{1}{52} \leq \varepsilon \leq \frac{1}{25}$, we have $\lim _{n \rightarrow \infty} q_{k_{n}}\left\|q_{k_{n}} \alpha\right\|=\varepsilon$. The set $\Lambda$ is of full Lebesgue measure. Indeed, the Gauss map $G(x)=\left\{\frac{1}{x}\right\}$ is mixing for the absolutely continuous measure with density $\frac{1}{\ln 2} \frac{1}{1+x}$, hence in particular $G^{2}$ is ergodic. For any irrational $\alpha \in \mathbb{R} / \mathbb{Z}$, let $\left\{a_{n}\right\}_{n \in \mathbb{N}}$ be the sequence of partial quotients of $\alpha$. Then we have (see [16])

$$
\frac{1}{2} \frac{1}{a_{n+1}+1}<q_{n}\left\|q_{n} \alpha\right\|<\frac{1}{a_{n+1}} .
$$

Recall that for any $m \in \mathbb{N}, G^{n}(\alpha) \in\left(\frac{1}{m+1}, \frac{1}{m}\right]$ iff $a_{n}=m$. Hence by ergodicity of $G^{2}$, for almost every $\alpha \in[0,1), a_{n+1}=25$ for infinitely many odd numbers $n$. Thus we obtain the claim.

Fix $\alpha \in \Lambda$. Recall that for every $n \in \mathbb{N}, \mathbb{R} / \mathbb{Z}$ is covered by a pair of towers

$$
\left\{T_{\alpha}^{i}\left[-\left\|q_{k_{n}} \alpha\right\|, 0\right)\right\}_{i=0, \ldots, q_{k_{n}-1}-1} \quad \text { and } \quad\left\{T_{\alpha}^{i}\left[0,\left\|q_{k_{n}-1} \alpha\right\|\right)\right\}_{i=0, \ldots, q_{k_{n}-1}}
$$

Note that $1-\alpha \in T_{\alpha}^{q_{k_{n}-1}}\left[0,\left\|q_{k_{n}-1} \alpha\right\|\right)$. More precisely, $1-\alpha=T_{\alpha}^{q_{k_{n}}-1}(0)+\left\|q_{k_{n}} \alpha\right\|$. Since $\left\{q_{k_{n}}\right\}_{n \in \mathbb{N}}$ is a rigidity sequence and $f$, regardless of the choice of discontinuity points, is of bounded variation, (86) follows from Proposition 7.5 and Koksma-Denjoy inequality:

$$
\left|f^{\left(q_{k_{n}}\right)}(x)-b_{n}\right| \leq \operatorname{Var}(f) \text { for every } x \in[0,1) .
$$

In view of Proposition 7.5 taking a subsequence if necessary, we get

$$
P=\lim _{n \rightarrow \infty}\left(f^{\left(2 q_{k_{n}}\right)}-2 b_{n}, f^{\left(q_{k_{n}}\right)}-b_{n}\right)_{*} L e b
$$

and

$$
Q=\lim _{n \rightarrow \infty}\left(f^{\left(-2 q_{k_{n}}\right)}+2 b_{n}, f^{\left(-q_{k_{n}}\right)}+b_{n}\right)_{*} L e b .
$$


By applying $\xi_{*}$ to both expressions we obtain

and

$$
\xi_{*} P=\lim _{n \rightarrow \infty}\left(\sum_{i=0}^{q_{k_{n}}-1}\left(f \circ T_{\alpha}^{q_{k_{n}}+i}-f \circ T_{\alpha}^{i}\right)\right)_{*} L e b
$$

$$
\xi_{*} Q=\lim _{n \rightarrow \infty}\left(\sum_{i=1}^{q_{k_{n}}}\left(f \circ T_{\alpha}^{-q_{k_{n}}-i}-f \circ T_{\alpha}^{-i}\right)\right)_{*} L e b .
$$

By using the invariance of the Lebesgue measure under $T_{\alpha}^{2 q_{k_{n}}}$, we get $\xi_{*} Q=(-\xi)_{*} P$.

Consider the sequence of pairs of disjoint Rokhlin towers

$$
V_{n}:=\bigcup_{i=0}^{q_{k_{n}}-1} T_{\alpha}^{i}\left[3\left\|q_{k_{n}} \alpha\right\|, \frac{1}{3}\left\|q_{k_{n}-1} \alpha\right\|\right) \text { and } W_{n}:=\bigcup_{i=0}^{q_{k_{n}}-1} T_{\alpha}^{i}\left[\frac{2}{3}\left\|q_{k_{n}-1} \alpha\right\|,\left\|q_{k_{n}-1} \alpha\right\|-3\left\|q_{k_{n}} \alpha\right\|\right) \text {. }
$$

We know (see e.g. [16]) that

Hence

$$
\frac{1}{2 q_{k_{n}}}<\left\|q_{k_{n}-1} \alpha\right\|<\frac{1}{q_{k_{n}}}
$$

$$
\frac{\left\|q_{k_{n}-1} \alpha\right\|}{\left\|q_{k_{n}} \alpha\right\|}<\frac{1}{q_{k_{n}}\left\|q_{k_{n}} \alpha\right\|} \rightarrow \frac{1}{\varepsilon} \text { and } \frac{\left\|q_{k_{n}-1} \alpha\right\|}{\left\|q_{k_{n}} \alpha\right\|}>\frac{1}{2 q_{k_{n}}\left\|q_{k_{n}} \alpha\right\|} \rightarrow \frac{1}{2 \varepsilon}
$$

By using the fact that $\varepsilon<\frac{1}{25}$, for sufficiently large $n$ we obtain that

$$
12<\frac{\left\|q_{k_{n}-1} \alpha\right\|}{\left\|q_{k_{n}} \alpha\right\|}<53 .
$$

In view of 88 we get

$$
\operatorname{Leb}\left(W_{n}\right)=\operatorname{Leb}\left(V_{n}\right)=q_{k_{n}}\left(\frac{1}{3}\left\|q_{k_{n}-1} \alpha\right\|-3\left\|q_{k_{n}} \alpha\right\|\right)>q_{k_{n}}\left\|q_{k_{n}} \alpha\right\|>\frac{1}{53},
$$

for sufficiently large $n \in \mathbb{N}$, that is the measures of $V_{n}, W_{n}$ are bounded away from 0 .

By the remark to Lemma 3.4 in [15], this implies that, for almost every $\beta_{1} \in[0,1)$, there exists an infinite set $N_{1}$ of natural numbers such that $\beta_{1} \in V_{n}$ for each $n \in N_{1}$. Once such $\beta_{1}$ and $N_{1}$ are fixed, the same argument yields that for almost every $\beta_{2} \in[0,1)$ there exists an infinite subset $N_{2} \subset N_{1}$ such that $\beta_{2} \in W_{n}$ for each $n \in N_{2}$. Using Fubini's theorem, we get that for almost every $\left(\beta_{1}, \beta_{2}\right) \in[0,1) \times[0,1)$ there exist infinitely many integers $n$ such that $\beta_{1} \in V_{n}$ and $\beta_{2} \in W_{n}$. Let $D_{\alpha}$ be the set of such pairs $\left(\beta_{1}, \beta_{2}\right)$.

Now we fix $\left(\beta_{1}, \beta_{2}\right)$ in $D_{\alpha}$. Extracting a subsequence if necessary, we may assume that $\beta_{1} \in V_{n}$ and $\beta_{2} \in W_{n}$ for all $n$. Since $V_{n}$ and $W_{n}$ are disjoint and $0,1-\alpha \notin W_{n} \cup V_{n}$ for all $n \in \mathbb{N}$, the points $0,1-\alpha, \beta_{1}$ and $\beta_{2}$ are distinct. Note that, by the choice of $W_{n}$ and $V_{n}$ the towers $\bigcup_{i=0}^{q_{k_{n}}-1} T^{-i}\left[\beta_{1}, \beta_{1}+\left\|q_{k_{n}} \alpha\right\|\right), \bigcup_{i=0}^{q_{k_{n}}-1} T^{-i}\left[\beta_{2}, \beta_{2}+\left\|q_{k_{n}} \alpha\right\|\right)$ and $\bigcup_{i=0}^{q_{k_{n}}} T^{-n}\left[0,\left\|q_{k_{n}} \alpha\right\|\right)$ are pairwise disjoint. Indeed, we have the following inclusions

$$
\begin{gathered}
\bigcup_{i=0}^{q_{k_{n}}-1} T^{-i}\left[\beta_{1}, \beta_{1}+\left\|q_{k_{n}} \alpha\right\|\right) \subset \bigcup_{i=0}^{q_{k_{n}}-1} T_{\alpha}^{i}\left[3\left\|q_{k_{n}} \alpha\right\|, \frac{1}{3}\left\|q_{k_{n}-1} \alpha\right\|+\left\|q_{k_{n}} \alpha\right\|\right), \\
\bigcup_{i=0}^{q_{k_{n}}-1} T^{-i}\left[\beta_{2}, \beta_{2}+\left\|q_{k_{n}} \alpha\right\|\right) \subset \bigcup_{i=0}^{q_{k_{n}}-1} T_{\alpha}^{i}\left[\frac{2}{3}\left\|q_{k_{n}-1} \alpha\right\|,\left\|q_{k_{n}-1} \alpha\right\|-2\left\|q_{k_{n}} \alpha\right\|\right),
\end{gathered}
$$

and

$$
\bigcup_{i=0}^{q_{k_{n}}} T^{-i}\left[0,\left\|q_{k_{n}} \alpha\right\|\right) \subset \bigcup_{i=0}^{q_{k_{n}}-1} T_{\alpha}^{i}\left[0,2\left\|q_{k_{n}} \alpha\right\|\right) .
$$

In view of 88 , we get $\frac{1}{3}\left\|q_{k_{n}-1} \alpha\right\|>4\left\|q_{k_{n}} \alpha\right\|$ for sufficiently large $n$. In particular

$$
\frac{1}{3}\left\|q_{k_{n}-1} \alpha\right\|+\left\|q_{k_{n}} \alpha\right\|<\frac{2}{3}\left\|q_{k_{n}-1} \alpha\right\|,
$$


which shows that the intervals

$$
\left[0,2\left\|q_{k_{n}} \alpha\right\|\right), \quad\left[3\left\|q_{k_{n}} \alpha\right\|, \frac{1}{3}\left\|q_{k_{n}-1} \alpha\right\|+\left\|q_{k_{n}} \alpha\right\|\right) \text { and }\left[\frac{2}{3}\left\|q_{k_{n}-1} \alpha\right\|,\left\|q_{k_{n}-1} \alpha\right\|-2\left\|q_{k_{n}} \alpha\right\|\right)
$$

are pairwise disjoint. This implies the desired disjointness of the aforementioned towers. This allow us to control the atoms of limit measures and their respective masses.

Suppose now that $f$ has discontinuity points at $0,1-\alpha, \beta_{1}$ and $\beta_{2}$, where $\left(\beta_{1}, \beta_{2}\right) \in D_{\alpha}$ with jumps $d_{0}, d_{1-\alpha}, d_{\beta_{1}}$ and $d_{\beta_{2}}$ respectively. Since $k_{n}$ is odd, in view of Remark 7.6 the expression $\sum_{i=0}^{q_{k_{n}}-1}\left(f \circ T_{\alpha}^{q_{k_{n}}+i}(x)-f \circ T_{\alpha}^{i}(x)\right)$ may only take non-zero values for $x$ on towers $U_{1}:=$ $\bigcup_{i=0}^{q_{k_{n}}-1} T^{-i}\left[0,\left\|q_{k_{n}} \alpha\right\|\right), U_{2}:=\bigcup_{i=0}^{q_{k_{n}}-1} T^{-i}\left[1-\alpha, 1-\alpha+\left\|q_{k_{n}} \alpha\right\|\right), U_{3}:=\bigcup_{i=0}^{q_{k_{n}}-1} T^{-i}\left[\beta_{1}, \beta_{1}+\right.$ $\left.\left\|q_{k_{n}} \alpha\right\|\right)$ and $U_{4}:=\bigcup_{i=0}^{q_{k_{n}}-1} T^{-i}\left[\beta_{2}, \beta_{2}+\left\|q_{k_{n}} \alpha\right\|\right)$. We have proved though, that $U_{3}$ is disjoint with other towers and the same is true for $U_{4}$. Thus we get that

$$
\sum_{i=0}^{q_{k_{n}}-1}\left(f \circ T_{\alpha}^{q_{k_{n}}+i}(x)-f \circ T_{\alpha}^{i}(x)\right)=-d_{\beta_{1}} \text { for } x \in U_{3},
$$

and

On the other hand,

$$
\sum_{i=0}^{q_{k_{n}}-1}\left(f \circ T_{\alpha}^{q_{k_{n}}+i}(x)-f \circ T_{\alpha}^{i}(x)\right)=-d_{\beta_{1}} \text { for } x \in U_{4} .
$$

$$
U_{1} \cap U_{2}=\bigcup_{i=1}^{q_{k_{n}}-1} T^{-i}\left[0,\left\|q_{k_{n}} \alpha\right\|, U_{1} \backslash U_{2}=\left[0,\left\|q_{k_{n}} \alpha\right\|\right) \text {, and } U_{2} \backslash U_{1}=\left[\left\|q_{k_{n}} \alpha\right\|, 2\left\|q_{k_{n}} \alpha\right\|\right) .\right.
$$

Hence

$$
\begin{gathered}
\sum_{i=0}^{q_{k_{n}}-1}\left(f \circ T_{\alpha}^{q_{k_{n}}+i}(x)-f \circ T_{\alpha}^{i}(x)\right)=-d_{0}-d_{1-\alpha} \text { for } x \in U_{1} \cap U_{2}, \\
\sum_{i=0}^{q_{k_{n}}-1}\left(f \circ T_{\alpha}^{q_{k_{n}}+i}(x)-f \circ T_{\alpha}^{i}(x)\right)=-d_{0} \text { for } x \in\left[0,\left\|q_{k_{n}} \alpha\right\|\right),
\end{gathered}
$$

and

Finally

$$
\sum_{i=0}^{q_{k_{n}}-1}\left(f \circ T_{\alpha}^{q_{k_{n}}+i}(x)-f \circ T_{\alpha}^{i}(x)\right)=-d_{1-\alpha} \text { for } x \in\left[\left\|q_{k_{n}} \alpha\right\|, 2\left\|q_{k_{n}} \alpha\right\|\right) .
$$

$$
\sum_{i=0}^{q_{k_{n}}-1}\left(f \circ T_{\alpha}^{q_{k_{n}}+i}(x)-f \circ T_{\alpha}^{i}(x)\right)=0 \text { for } x \notin U_{1} \cup U_{2} \cup U_{3} \cup U_{4} .
$$

Note that $-d_{0}-d_{1-\alpha}=d_{\beta_{1}}+d_{\beta_{2}}$, since the sum of jumps of $f$ is 0 . Moreover

and

$$
\begin{gathered}
\operatorname{Leb}\left(\left[0,\left\|q_{k_{n}} \alpha\right\|\right)\right)=\operatorname{Leb}\left(\left[\left\|q_{k_{n}} \alpha\right\|, 2\left\|q_{k_{n}} \alpha\right\|\right)\right)=\left\|q_{k_{n}} \alpha\right\| \rightarrow 0, \\
\operatorname{Leb}\left(U_{3}\right)=\operatorname{Leb}\left(U_{4}\right)=q_{k_{n}}\left\|q_{k_{n}} \alpha\right\| \rightarrow \varepsilon
\end{gathered}
$$

$$
\operatorname{Leb}\left(U_{1} \cap U_{2}\right)=q_{k_{n}}\left\|q_{k_{n}} \alpha\right\|-\left\|q_{k_{n}} \alpha\right\| \rightarrow \varepsilon .
$$

Hence we get that $\xi_{*} P=\lim _{n \rightarrow \infty}\left(\sum_{i=0}^{q_{k_{n}}-1} f \circ T_{\alpha}^{q_{k_{n}}+i}-f \circ T_{\alpha}^{i}\right)_{*}$ Leb is a measure such that $\xi_{*} P\left(\left\{-d_{\beta_{1}}\right\}\right)=\xi_{*} P\left(\left\{-d_{\beta_{2}}\right\}\right)=\xi_{*} P\left(\left\{d_{\beta_{1}}+d_{\beta_{2}}\right\}\right)=\varepsilon$ and $\xi_{*} P(\{0\})=1-3 \varepsilon$. Since $\xi_{*} Q=$ $(-\xi)_{*} P$, we obtain the final claim.

We now state a reformulation of the above lemma for rotations on arbitrary large circles. If needed, throughout the proof of this lemma, we identify $\mathbb{R} / x \mathbb{Z}$ with $[0, x)$ for every $x \in \mathbb{R}_{>0}$.

Lemma 7.8. There exists a subset $\Delta_{0} \subset \Delta:=\left\{(x, y) \in \mathbb{R}_{>0}^{2} ; 0<y<x\right\}$ of full Lebesgue measure in $\Delta$ with the property that for every $(l, \alpha) \in \Delta_{0}$ there exists a set $D_{l, \alpha} \subset(\mathbb{R} / l \mathbb{Z}) \times$ $(\mathbb{R} / l \mathbb{Z})$ of full Lebesgue measure such that for every $\left(\beta_{1}, \beta_{2}\right) \in D_{l, \alpha}$ we have 
- the numbers $0, l-\alpha, \beta_{1}$ and $\beta_{2}$ are distinct in $\mathbb{R} / l \mathbb{Z}$ and

- if $T_{\alpha}$ is the rotation on $\mathbb{R} / l \mathbb{Z}$ by $\alpha \in \mathbb{R} / l \mathbb{Z}$ and $h: \mathbb{R} / l \mathbb{Z} \rightarrow \mathbb{R}_{>0}$ is a piecewise constant function with exactly 4 discontinuity points at $0, l-\alpha, \beta_{1}, \beta_{2}$ and rationally independent jumps at $\beta_{1}$ and $\beta_{2}$, then the special flow $T_{\alpha}^{h}$ is weakly mixing and disjoint with its inverse.

Proof. Let $\Lambda \subset[0,1)$ and $D_{\alpha} \subset[0,1) \times[0,1)$ for $\alpha \in \Lambda$ be the sets given by Lemma 7.7. For any $l \in \mathbb{R}_{>0}$, let us also denote by $l:[0,1) \rightarrow[0, l)$ the map given by $l(x):=l x$. We also consider $l$ as a map between $\mathbb{R} / \mathbb{Z}$ and $\mathbb{R} / l \mathbb{Z}$. For any $l \in \mathbb{R}_{>0}$ let $\Lambda_{l}:=l(\Lambda) \subset[0, l)$ and set $D_{l, \alpha}:=(l \times l)\left(D_{l^{-1} \alpha}\right) \subset[0, l) \times[0,1)$ for any $\alpha \in[0, l)$. Define $\Delta_{0}:=\left\{(x, y) ; x \in \mathbb{R}_{>0}, y \in \Lambda_{x}\right\}$. Note that $\Delta_{0}$ is of full Lebesgue measure in $\Delta$ and for every $l \in \mathbb{R}_{>0}$ and $\alpha \in \Lambda_{l}$ the set $D_{l, \alpha}$ is of full Lebesgue measure in $(\mathbb{R} / l \mathbb{Z}) \times(\mathbb{R} / l \mathbb{Z})$.

Take $(l, \alpha) \in \Delta_{0}$ and $\left(\beta_{1}, \beta_{2}\right) \in D_{l, \alpha}$. By the definition of $\Lambda$ and $D_{l^{-1} \alpha}$, the points 0 , $l-\alpha, \beta_{1}$ and $\beta_{2}$ are distinct. Let $h: \mathbb{R} / l \mathbb{Z} \rightarrow \mathbb{R}_{>0}$ be a piecewise constant function which has exactly 4 discontinuity points at $0, l-\alpha, \beta_{1}$ and $\beta_{2}$. Assume that the jumps $d_{\beta_{1}}$ and $d_{\beta_{2}}$ at $\beta_{1}$ and $\beta_{2}$ are rationally independent. Consider the special flow $T_{\alpha}^{h}$ on $[0, l)^{h}$. The map $\left(l^{-1} \times I d\right):[0, l)^{h} \rightarrow[0,1)^{h \circ l}$ establishes an isomorphism of flows $T_{\alpha}^{h}$ and $T_{l^{-1} \alpha}^{h \circ l}$. The roof function $h \circ l$ has discontinuities at $0,1-l^{-1} \alpha, l^{-1} \beta_{1}$ and $l^{-1} \beta_{2}$ and has jumps $d_{\beta_{1}}$ and $d_{\beta_{2}}$ at $l^{-1} \beta_{1}$ and $l^{-1} \beta_{2}$ respectively. Moreover, $l^{-1} \alpha \in \Lambda$ and $\left(l^{-1} \beta_{1}, l^{-1} \beta_{2}\right) \in D_{\alpha}$. In view of Lemma 7.7. this gives

$$
\operatorname{Leb}_{2 b_{n}, b_{n}}^{h \circ l} \rightarrow \int_{\mathbb{R}^{2}} \operatorname{Leb}_{-t,-u}^{h \circ l} d P(t, u), \quad \text { and } \operatorname{Leb}_{-2 b_{n},-b_{n}}^{h \circ l} \rightarrow \int_{\mathbb{R}^{2}} \operatorname{Leb}_{-t,-u}^{h \circ l} d Q(t, u) \text { weakly, }
$$

for some increasing to infinity real sequence $\left\{b_{n}\right\}_{n \in \mathbb{N}}$ and measures $P, Q \in \mathcal{P}\left(\mathbb{R}^{2}\right)$. Furthermore, $\xi_{*} P$ is atomic and has atoms at $0,-d_{\beta_{1}},-d_{\beta_{2}}$ and $d_{\beta_{1}}+d_{\beta_{1}}$, while $\xi_{*} Q$ is also atomic and has atoms at $0, d_{\beta_{1}}, d_{\beta_{2}}$ and $-\left(d_{\beta_{1}}+d_{\beta_{1}}\right)$. Since $d_{\beta_{1}}$ and $d_{\beta_{2}}$ are rationally independent, Proposition 3.5 implies that $T_{l^{-1} \alpha}^{h \circ l}$ is weakly mixing. Moreover, the rational independence of $d_{\beta_{1}}$ and $d_{\beta_{2}}$ also gives $\xi_{*} P \neq \xi_{*} Q$ which yields $P \neq Q$. In view of Corollary 3.4 this gives that $T_{l^{-1} \alpha}^{h \circ l}$ is disjoint with its inverse. Since $T_{l^{-1} \alpha}^{h \circ l}$ and $T_{\alpha}^{h}$ are isomorphic, $T_{\alpha}^{h}$ is weakly mixing and disjoint with its inverse.

We are now ready to give the proof of the main result of this paper.

Proof of Theorem 1.1. In view of Corollary 7.3, the set of translation structures whose associated vertical flow is weakly mixing and disjoint with its inverse is a $G_{\delta}$ set in every connected component of the moduli space. We now show that there is a dense subset of translation structures in each non-hyperelliptic connected component $C$ so that the associated vertical flows are weakly mixing and disjoint with their inverses.

Fix a non-hyperelliptic connected component $C$ of the moduli space. Recall that for some $d \geq 2$ and an alphabet $\mathcal{A}$ of $d$ elements, there is a permutation $\pi=\left(\pi_{0}, \pi_{1}\right) \in S_{0}^{\mathcal{A}}$ in the extended Rauzy class associated with $C$, such that

$$
\pi_{1}\left(\pi_{0}^{-1}(1)\right)=d \quad \text { and } \quad \pi_{1}\left(\pi_{0}^{-1}(d)\right)=1 .
$$

Let $\Omega:=\Omega_{\pi}$ be the translation matrix corresponding to $\pi$. Then, in view of Corollary 4.2 there exist symbols $a_{1}, a_{2} \in \mathcal{A}$ such that

$$
\Omega_{a_{1} a_{2}}=\Omega_{a_{2} a_{1}}=0
$$

and the numbers

$$
(\Omega \tau)_{a_{2}}-(\Omega \tau)_{a_{1}} \text { and }(\Omega \tau)_{a_{i}}-\left((\Omega \tau)_{\pi_{0}^{-1}(1)}+(\Omega \tau)_{\pi_{0}^{-1}(d)}\right)
$$

are rationally independent for $i=1,2$ whenever $\tau$ is rationally independent.

Let

$$
\Xi_{*}:=\left\{(\pi, \lambda, \tau) \in \Theta_{\pi} ; \lambda_{a}=0 \text { for } a \in \mathcal{A} \backslash\left\{\pi_{0}^{-1}(1), \pi_{0}^{-1}(d), a_{1}, a_{2}\right\}\right\}
$$

Let $C_{*}:=\left\{M(\pi, \lambda, \tau) \in C ;(\pi, \lambda, \tau) \in \Xi_{*}\right\}$. In view of Lemma 7.4 this is a dense subset of $C$. Hence to prove the density of the desired property in $C$, it is enough to prove that this property 
holds for a dense set in $C_{*}$. We prove this by finding a dense subset of parameters in $\Xi_{*}$ such that the associated translation structures have the sought properties.

Note that the set $\Xi \subset \Xi_{*}$ given by

$$
\Xi:=\left\{(\pi, \lambda, \tau) \in \Xi_{*} ; T_{\pi, \lambda} \text { is ergodic; } \lambda_{\pi_{0}^{-1}(1)} \neq \lambda_{\pi_{0}^{-1}(d)} ; \tau \text { is rationally independent }\right\}
$$

is dense in $\Xi_{*}$. Let $\zeta=M(\pi, \lambda, \tau) \in C_{*}$ with $(\pi, \lambda, \tau) \in \Xi$. Let $\mathcal{T}^{\zeta}$ be the corresponding vertical flow. Recall that it has a special representation $T_{\pi, \lambda}^{h}$ over the IET $T_{\pi, \lambda}:[0,|\lambda|) \rightarrow[0,|\lambda|)$ and under a piecewise constant roof function $h:[0,|\lambda|) \rightarrow \mathbb{R}_{>0}$ which is constant over exchanged intervals. Moreover, if we consider $h=\left\{h_{a}\right\}_{a \in \mathcal{A}}$ as a vector of values, where $h_{a}$ is the value of $h$ over the interval corresponding to $a$, then $h_{a}=-(\Omega \tau)_{a}$. However, since $(\pi, \lambda, \tau) \in \Xi_{*}$, we have that $\lambda_{a}=0$ for $a \in \mathcal{A} \backslash\left\{\pi_{0}^{-1}(1), \pi_{0}^{-1}(d), a_{1}, a_{2}\right\}$. Thus we can reduce the data describing the above special representation.

Let $\hat{\pi}=\left(\hat{\pi}_{0}, \hat{\pi}_{1}\right)$ be the permutation on the alphabet $\hat{\mathcal{A}}:=\left\{\pi_{0}^{-1}(1), \pi_{0}^{-1}(d), a_{1}, a_{2}\right\}$ given by

$$
\hat{\pi}_{0}\left(\pi_{0}^{-1}(1)\right)=1, \quad \hat{\pi}_{0}\left(\pi_{0}^{-1}(d)\right)=4, \quad \hat{\pi}_{0}\left(a_{1}\right)=2, \quad \hat{\pi}_{0}\left(a_{2}\right)=3
$$

and

$$
\hat{\pi}_{1}\left(\pi_{0}^{-1}(1)\right)=4, \quad \hat{\pi}_{1}\left(\pi_{0}^{-1}(d)\right)=1, \quad \hat{\pi}_{1}\left(a_{1}\right)=2, \quad \hat{\pi}_{1}\left(a_{2}\right)=3 .
$$

For $a \in \hat{\mathcal{A}}$ let $\hat{\lambda}_{a}:=\lambda_{a}$. Moreover, since the intervals corresponding to $a \in \mathcal{A} \backslash \hat{\mathcal{A}}$ are empty, $h$ can be considered as a vector $\left\{h_{a}\right\}_{a \in \hat{\mathcal{A}}}$. Then $\mathcal{T}^{\zeta}$ has a special representation $T_{\hat{\pi}, \hat{\lambda}}^{h}$ over the $\operatorname{IET} T_{\hat{\pi}, \hat{\lambda}}:[0,|\lambda|) \rightarrow[0,|\lambda|)$.

Consider the sets $\Xi_{0}, \Xi_{1} \subset \Xi$ given by

$$
\Xi_{0}:=\left\{(\pi, \lambda, \tau) \in \Xi ; \lambda_{\pi_{0}^{-1}(1)}>\lambda_{\pi_{0}^{-1}(d)}\right\} \text { and } \Xi_{1}:=\left\{(\pi, \lambda, \tau) \in \Xi ; \lambda_{\pi_{0}^{-1}(1)}<\lambda_{\pi_{0}^{-1}(d)}\right\} .
$$

We have $\Xi_{0} \cup \Xi_{1}=\Xi$. Suppose first that $(\pi, \lambda, \tau) \in \Xi_{0}$ that is $\lambda_{\pi_{0}^{-1}(1)}>\lambda_{\pi_{0}^{-1}(d)}$. Let $\phi:\left\{(x, y, z, v) \in \mathbb{R}_{>0}^{4} ; x>v\right\} \rightarrow \mathbb{R}_{>0}^{4}$ be the diffeomorphism given by

$$
\phi(x, y, z, v):=(x-v, v, y, z) .
$$

Then after one step of the polygonal right hand side Rauzy-Veech induction on $\mathcal{T}_{\hat{\pi}, \hat{\lambda}}^{h}$ we get a special flow $T_{\alpha}^{\hat{h}}$ over the rotation $T_{\alpha}:\left[0, \hat{\lambda}_{\pi_{0}^{-1}(1)}+\hat{\lambda}_{a_{1}}+\hat{\lambda}_{a_{2}}\right) \rightarrow\left[0, \hat{\lambda}_{\pi_{0}^{-1}(1)}+\hat{\lambda}_{a_{1}}+\hat{\lambda}_{a_{2}}\right)$ by $\alpha=$ $\alpha(\hat{\lambda}):=\hat{\lambda}_{a_{1}}+\hat{\lambda}_{a_{2}}+\hat{\lambda}_{\pi_{0}^{-1}(d)}$ under a piecewise constant function $\hat{h}:\left[0, \hat{\lambda}_{\pi_{0}^{-1}(1)}+\hat{\lambda}_{a_{1}}+\hat{\lambda}_{a_{2}}\right) \rightarrow \mathbb{R}_{>0}$ with values $h_{\pi_{0}^{-1}(1)}, h_{\pi_{0}^{-1}(1)}+h_{\pi_{0}^{-1}(d)}, h_{a_{1}}, h_{a_{2}}$ over the consecutive intervals of lengths given by the vector $\phi\left(\hat{\lambda}_{\pi_{0}^{-1}(1)}, \hat{\lambda}_{a_{1}}, \hat{\lambda}_{a_{2}}, \hat{\lambda}_{\pi_{0}^{-1}(d)}\right)$. Recall that the flows $\mathcal{T}_{\hat{\pi}, \hat{\lambda}}^{h}$ and $T_{\alpha}^{\hat{h}}$ are isomorphic. Let $l=l(\hat{\lambda}):=\hat{\lambda}_{\pi_{0}^{-1}(1)}+\hat{\lambda}_{a_{1}}+\hat{\lambda}_{a_{2}}, \beta_{1}=\beta_{1}(\hat{\lambda}):=\hat{\lambda}_{\pi_{0}^{-1}(1)}$ and $\beta_{2}=\beta_{2}(\hat{\lambda}):=\hat{\lambda}_{\pi_{0}^{-1}(1)}+\hat{\lambda}_{a_{1}}$. Then $\hat{h}:[0, l) \rightarrow \mathbb{R}_{>0}$ has discontinuities at points $l-\alpha, \beta_{1}$ and $\beta_{2}$. The jump at the point $\beta_{1}$ is equal to $h_{a_{1}}-\left(h_{\pi_{0}^{-1}(1)}+h_{\pi_{0}^{-1}(d)}\right)$, while at the point $\beta_{2}$ equals $h_{a_{2}}-h_{a_{1}}$. Moreover, we have

$$
h_{a_{1}}-\left(h_{\pi_{0}^{-1}(1)}+h_{\pi_{0}^{-1}(d)}\right)=-(\Omega \tau)_{a_{1}}+\left((\Omega \tau)_{\pi_{0}^{-1}(1)}+(\Omega \tau)_{\pi_{0}^{-1}(d)}\right)
$$

and

$$
h_{a_{2}}-h_{a_{1}}=-(\Omega \tau)_{a_{2}}+(\Omega \tau)_{a_{2}} .
$$

Since $\tau$ is a rationally independent vector, Corollary 4.2 yields the rational independence of the jumps at $\beta_{1}$ and $\beta_{2}$. From now on we treat $T_{\alpha}$ as a rotation on $\mathbb{R} / l \mathbb{Z}$. Furthermore, we also treat $\hat{h}$ as a piecewise constant function on $\mathbb{R} / l \mathbb{Z}$. Then $\hat{h}: \mathbb{R} / l \mathbb{Z} \rightarrow \mathbb{R}_{>0}$ gets an additional discontinuity point at 0 .

Let us consider the diffeomorphism $\psi: \mathbb{R}_{>0}^{4} \rightarrow\left\{(x, y, z, v) \in \mathbb{R}_{>0}^{4} ; 0<x-y<z<v<x\right\}$ given by

$$
\psi(x, y, z, v):=(x+y+z+v, y+z+v, x+y, x+y+z) .
$$

Then

$$
\psi \circ \phi:\left\{(x, y, z, v) \in \mathbb{R}_{>0}^{4} ; x>v\right\} \rightarrow\left\{(x, y, z, v) \in \mathbb{R}_{>0}^{4} ; 0<x-y<z<v<x\right\}
$$

is a diffeomorphism and $\psi \circ \phi(\hat{\lambda})=\left(l, \alpha, \beta_{1}, \beta_{2}\right)$. 
Let $\Delta_{0} \subset\left\{(x, y) \in \mathbb{R}_{>0}^{2} ; y \in(0, x)\right\}$ and $D_{l, \alpha} \subset(\mathbb{R} / l \mathbb{Z}) \times(\mathbb{R} / l \mathbb{Z})$ for $(l, \alpha) \in \Delta_{0}$ be sets given by Lemma 7.8. Then, by Lemma 7.8. for every $(l, \alpha) \in \Delta_{0}$ and $\left(\beta_{1}, \beta_{2}\right) \in D_{l, \alpha}$, the special flow $T_{\alpha}^{\hat{h}}$ over the rotation by $\alpha$ on $\mathbb{R} / l \mathbb{Z}$ and under a piecewise constant roof function with discontinuity points $0, l-\alpha, \beta_{1}, \beta_{2}$ and with rationally independent jumps at $\beta_{1}$ and $\beta_{2}$ is weakly mixing and disjoint with its inverse. Consider

$$
\mathscr{G}:=\left\{(x, y, z, v) \in \mathbb{R}_{>0}^{4} ;(x, y) \in \Delta_{0}, \frac{y}{x} \in \mathbb{R} \backslash \mathbb{Q},(z, v) \in D_{x, y}, 0<x-y<z<v<x\right\} .
$$

In view of Lemma 7.8, $\Delta_{0}$ is dense in $\left\{(x, y) \in \mathbb{R}_{>0} ; y<x\right\}$ and $D_{x, y}$ is dense in $(0, x) \times(0, x)$. Therefore $\mathscr{G}$ is a dense set in $\left\{(x, y, z, v) \in \mathbb{R}_{>0}^{4} ; 0<x-y<z<v<x\right\}$. As $\psi \circ \phi$ is a diffeomorphism, the set $(\psi \circ \phi)^{-1}(\mathscr{G})$ is dense in $\left\{(x, y, z, v) \in \mathbb{R}_{>0}^{4} ; x>v\right\}$. Hence the set

$$
\Gamma_{0}:=\left\{(\pi, \lambda, \tau) \in \Xi_{0} ; \hat{\lambda} \in(\psi \circ \phi)^{-1}(\mathscr{G}) \text { and } \tau \text { is a rationally independent vector }\right\}
$$

is dense in $\Xi_{0}$. By going along the same lines and by using the left-hand side polygonal RauzyVeech induction, we find a dense set $\Gamma_{1} \subset \Xi_{1}$ which has analogous properties.

If $\gamma=(\pi, \lambda, \tau) \in \Gamma_{0} \cup \Gamma_{1}$ then $(l(\hat{\lambda}), \alpha(\hat{\lambda})) \in \Delta_{0},\left(\beta_{1}(\hat{\lambda}), \beta_{2}(\hat{\lambda})\right) \in D_{l(\hat{\lambda}), \alpha(\hat{\lambda})}$ and the vertical flow on $M(\gamma)$ is isomorphic to a special flow $T_{\alpha(\hat{\lambda})}^{\hat{h}}$ on $(\mathbb{R} / l(\hat{\lambda}) \mathbb{Z})^{\hat{h}}$, where $\hat{h}: \mathbb{R} / l(\hat{\lambda}) \mathbb{Z} \rightarrow \mathbb{R}_{>0}$ is a piecewise constant roof function with discontinuities at $0, l(\hat{\lambda})-\alpha(\hat{\lambda}), \beta_{1}(\hat{\lambda}), \beta_{2}(\hat{\lambda})$ and the jumps at $\beta_{1}(\hat{\lambda})$ and $\beta_{2}(\hat{\lambda})$ are rationally independent. In view of Lemma 7.8 those flows are weakly mixing and disjoint with their inverses. As $\Gamma_{0} \cup \Gamma_{1}$ is dense in $\Xi$, it is also dense in $\Xi_{*}$. Since $M: \Theta_{\pi} \rightarrow C$ given by $(\pi, \lambda, \tau) \mapsto M(\pi, \lambda, \tau)$ is continuous and $M\left(\Xi_{*}\right)=C_{*}$, we have that $M\left(\Gamma_{0} \cup \Gamma_{1}\right)$ is dense in $C_{*}$. Moreover, by Lemma 7.4. $C_{*}$ is dense in $C$ which yields the result.

\section{ACKNOWLEDGMENTS}

The authors would like to thank M. Lemańczyk for fruitful discussions and for proposing the main ideas used in section 3. We would also like to thank S. Gouezel for pointing out the article [11] and for giving some ideas used in section 6. P. Berk and K. Frączek are partially supported by NCN grant nr 2014/13/B/ST1/03153.

\section{REFERENCES}

[1] W. Ambrose, Representation of ergodic flows, Ann. of Math. (2) 42 (1941), 723-739.

[2] H. Anzai, On an example of a measure preserving transformation which is not conjugate to its inverse, Proc. Japan Acad. 27 (1951), 517-522.

[3] A. Avila, G. Forni, Weak mixing for interval exchange transformations and translation flows, Ann. of Math. (2) 165 (2007), 637-664.

[4] P. Berk, K. Frączek, On special flows that are not isomorphic to their inverses, Discrete Contin. Dyn. Syst. 35 (2015), 829-855.

[5] A.I. Danilenko, V.V. Ryzhikov, On self-similarities of ergodic flows, Proc. Lond. Math. Soc. 104 (2012), 431-454.

[6] A. del Junco, Disjointness of measure-preserving transformations, minimal self-joinings and category, Ergodic theory and dynamical systems, I (College Park, Md., 1979-80), pp. 81-89, Progr. Math., 10, Birkhäuser, Boston, Mass., 1981.

[7] R.H. Fox, R.B. Kershner, Concerning the transitive properties of geodesics on a rational polyhedron, Duke Math. J. 2 (1936), 147-150.

[8] K. Frączek, Density of mild mixing property for vertical flows of Abelian differentials, Proc. Amer. Math. Soc. 137 (2009), 4129-4142.

[9] K. Frączek, J. Kułaga-Przymus, M. Lemańczyk, Non-reversibility and self-joinings of higher orders for ergodic flows, J. Anal. Math. 122 (2014), 163-227.

[10] E. Glasner, Ergodic theory via joinings, Mathematical Surveys and Monographs, 101. American Mathematical Society, Providence, RI, 2003.

[11] C. Goffman, G. Pedrick, A proof of the homeomorphism of Lebesgue-Stieltjes measure with Lebesgue measure, Proc. Amer. Math. Soc. 52 (1975), 196-198.

[12] P.R. Halmos, Ergodic Theory, Chelsea, New York, 1956. 
[13] A.B. Katok, Interval exchange transformations and some special flows are not mixing, Israel J. Math. 35 (1980), 301-310.

[14] A.B. Katok, A.N. Zemljakov, Topological transitivity of billiards in polygons, Mat. Zametki 18 (1975), 291-300.

[15] J. King, Joining-rank and the structure of finite rank mixing transformations, J. Analyse Math. 51 (1988), $182-227$.

[16] A.Y. Khinchin, Continued fractions, The University of Chicago Press, Chicago-London, 1964.

[17] M. Kontsevich, A. Zorich, Connected components of the moduli spaces of Abelian differentials with prescribed singularities, Invent. Math. 153 (2003), 631-678.

[18] H. Masur, Interval exchange transformations and measured foliations, Ann. of Math. (2) 115 (1982), $169-200$

[19] J. Moser, On the volume elements on a manifold, Trans. Amer. Math. Soc. 120 (1965), $286-294$.

[20] V.V. Ryzhikov, Partial multiple mixing on subsequences can distinguish between automorphisms $T$ and $T^{-1}$, Math. Notes 74 (2003), 841-847.

[21] S.M. Srivastava, A course on Borel sets, Graduate Texts in Mathematics, 180. Springer-Verlag, New York, 1998.

[22] W.A. Veech, Gauss measures for transformations on the space of interval exchange maps, Ann. of Math. 115 (1982), 201-242.

[23] J.C. Yoccoz, Interval exchange maps and translation surface, Homogeneous flows, moduli spaces and arithmetic, 1-69, Clay Math. Proc., 10, Amer. Math. Soc., Providence, RI, 2010.

Faculty of Mathematics and Computer Science, Nicolaus Copernicus University, ul. Chopina

12/18, 87-100 TORuń, Poland

E-mail address: zimowy@mat.umk.pl

Faculty of Mathematics and Computer Science, Nicolaus Copernicus University, ul. Chopina

12/18, 87-100 Toruń, Poland

E-mail address: fraczek@mat.umk.pl

Laboratoire de Mathématiques Raphaël Salem, Normandie Université, Université de Rouen,

CNRS, Avenue de l'Université, 76801 Saint Etienne du Rouvray, France

E-mail address: Thierry.de-la-Rue@univ-rouen.fr 